\title{
Sex-specific differences in dispersal propensities and their consequences \\ for grey mouse lemurs (Microcebus murinus)
}

\author{
Dissertation \\ zur Erlangung des Doktorgrades \\ der Mathematisch-Naturwissenschaftlichen Fakultäten \\ der Georg-August-Universität zu Göttingen \\ vorgelegt von \\ Susanne Schliehe-Diecks \\ aus \\ Glandorf
}



Referent: Prof. Dr. Peter M. Kappeler Korreferent: Prof. Dr. Eckhard W. Heymann Tag der mündlichen Prüfung: 16.07.2012 

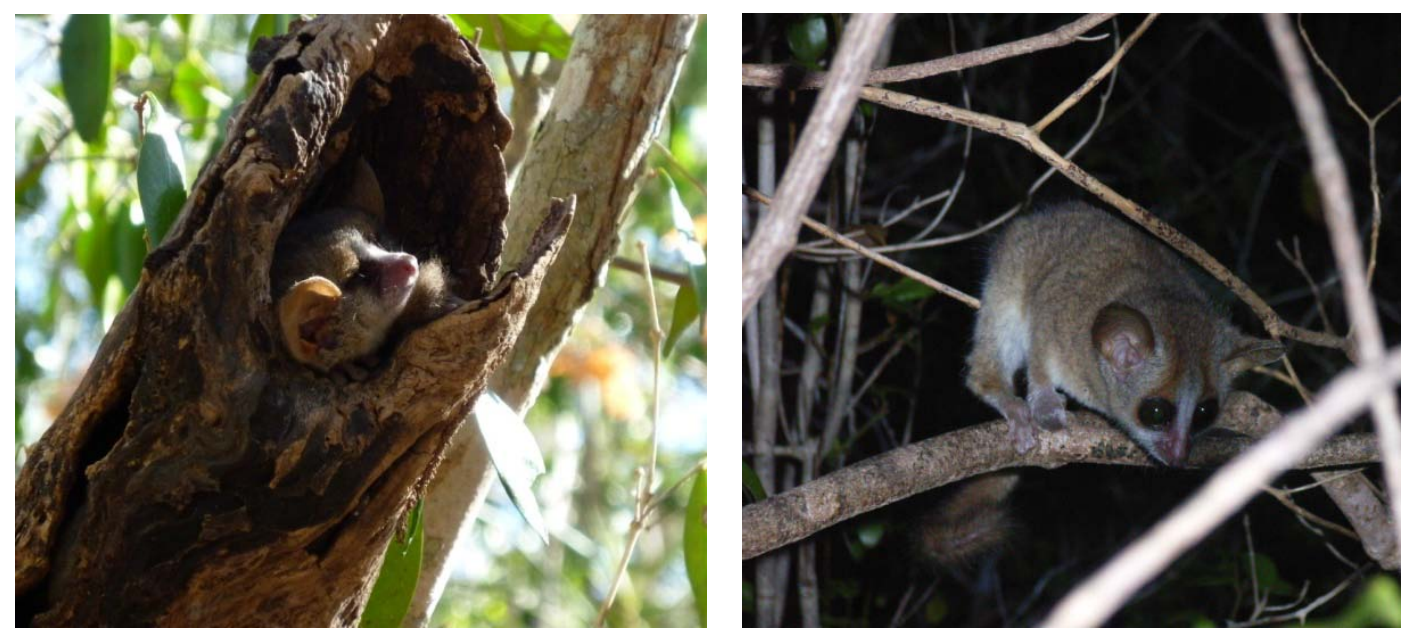

Grey mouse lemurs (Microcebus murinus),

nocturnal, solitary active primates 


\section{CONTENTS}

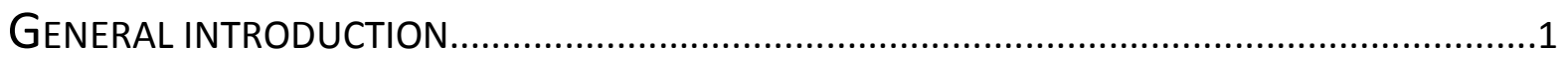

\section{CHAPTER 1: DISPERSAL MOVEMENTS}

Walk the line - Dispersal movements of gray mouse lemurs

with Manfred Eberle and Peter M. Kappeler

Behavioral Ecology \& Sociobiology (2012): in press

\section{CHAPTER 2: PROXIMATE MECHANISMS OF NATAL DISPERSAL}

Ready to go? On the relationship between natal dispersal and body mass in gray mouse lemurs (Microcebus murinus).

with Manfred Eberle and Peter M. Kappeler

Behavioral Ecology: submitted

\section{CHAPTER 3: SEX-SPECIFIC BEHAVIOURAL DIFFERENCES}

On the application of mixed hidden Markov models to multiple behavioural time series.

with Peter M. Kappeler and Roland Langrock

Interface Focus (2012) 2: 180-189

\section{CHAPTER 4: CONSEQUENCES OF NATAL DISPERSAL}

The ghost of inbreeding avoidance - Consequences of natal dispersal for inbreeding risk in a small solitary mammal, the grey mouse lemur.

with Cornelia Kraus, Elise Huchard, Manfred Eberle and Peter M. Kappeler For submission 


\section{CONTENTS}

SUMMARY.....

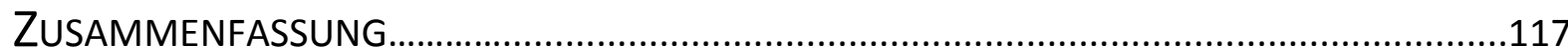

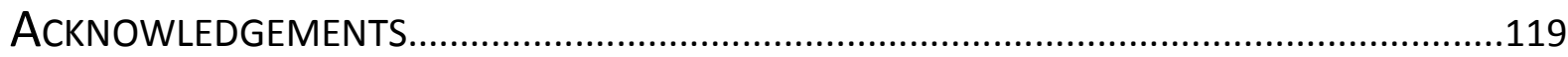

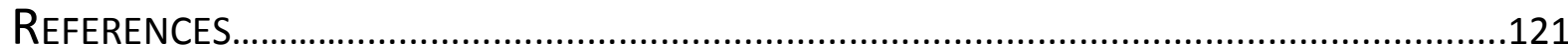

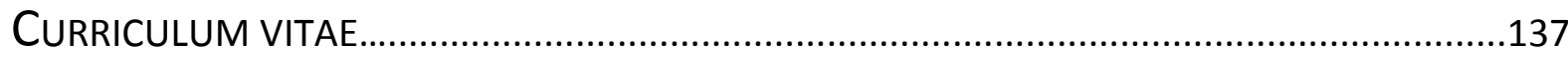




\section{GENERAL INTRODUCTION}

\section{DISPERSAL - A UBIQUITOUS PHENOMENON}

Dispersal is an almost ubiquitous life history event among animal taxa. The term describes the permanent movement (active or passive) of an organism or a propagule away from its site or group (of origin) to its first or subsequent (theoretical) breeding site or group (Shields 1987; Lidicker and Stenseth 1992; Clobert et al. 2009). In many species, this event occurs only once in a lifetime at a certain developmental stage, which has been labelled natal dispersal (Smale et al. 1997), but secondary transfer to other breeding units occurs in some taxa as well (Alberts and Altmann 1995a; Le Galliard et al. 2012). This mobility of individuals on a spatio-temporal scale makes dispersal a key process in ecology, evolution and conservation (Clobert et al. 2004; Nunes 2007). Dispersal also affects the distribution of genetic diversity by lowering the proportion of total diversity contained between populations (Wright 1969). It maintains genetic cohesion of a species across space, facilitates its global persistence despite local extinction, antagonizes genetic drift in small populations and allows the tracking of favourable environmental conditions when habitat quality or population sizes change stochastically (Ronce 2007; Clobert et al. 2009). Dispersal affects the evolution of local adaptation, speciation (Barton 2001), inbreeding depression (Pusey and Wolf 1996), cooperation and sociality (Moore 1992; Clutton-Brock and Lukas 2012) and community dynamics (Leibold et al. 2004). Understanding dispersal processes is therefore crucial to improve our understanding of evolution and for effective conservation of natural populations (Hansson 1992).

\section{EVOLUTION OF SEX-BASED DISPERSAL}

Despite the ubiquity of the phenomenon across species, the inclination to disperse and/or the travelled dispersal distances often differ between sexes. Sex-biased dispersal (SBD) has important consequences for the fitness of individuals, because both decisions to either go or stay entail costs and benefits. The potential costs for dispersers include poor habitat choice (Delibes et al. 2001), increased predation risk (Van Vuren and Armitage 1994; Alberts and Altmann 1995a; Yoder et al. 2004), separation from kin as potential coalition partners (Isbell and Van Vuren 1996), aggression by conspecifics (Smith 1987; Smale et al. 1997), missed 
reproductive opportunities (Alberts and Altmann 1995a), and decreased foraging efficiency because of unfamiliarity with the physical and social environment as well as increased energy expenditures through increased movement rates (Boinski et al. 2005; Bonte et al. 2012). Costs of philopatry can include reduction in reproductive success (De Sousa et al. 2009), risk of inbreeding (Lukas and Clutton-Brock 2011), competition with relatives (Kappeler and Fichtel 2012b) and aggression from conspecifics (Van Vuren and Armitage 1994). SBD changes not only the physical but also the social world and with it the formation of kinship structures and the opportunity for the evolution of cooperative behaviour (Moore 1992; Armitage et al. 2011; Lukas and Clutton-Brock 2011; Clutton-Brock and Lukas 2012; Van Noordwijk et al. 2012).

The general theoretical framework to explain the wide occurrence of SBD tendencies was set in the 1980s. In his review, Greenwood (1980) summarized important predominant patterns for birds and mammals and concluded that the direction of SBD was closely associated with the mating system. Female-biased dispersal was supposed to develop in systems of resource-defence monogamy, which is more typical for birds, whereas polygyny, a common breeding system of mammals, predisposed a species for male-biased dispersal. Since then, the arrival of molecular methods has facilitated the inference of SBD dramatically (Lawson Handley and Perrin 2007). With increasing number of studies the number of inconsistencies and exceptions from general patterns increased. Birds were found to be less monogamous than was previously thought (Petrie and Kempenaers 1998), and some mammals showed female-biased dispersal despite polygyny (reviewed in (Lawson Handley and Perrin 2007). The extent of SBD tendencies varies between species and in many cases there seems to be no strict dichotomy into habitually dispersing and habitually philopatric sexes. This is often true for species, in which reproduction is mainly restricted to a breeding pair, but also for species with polygynous mating systems (Armitage 1991; Strier 1994; McNutt 1996; Savage et al. 1996; Brockelman et al. 1998; Duarte et al. 2003; FernandezDuque 2009; Robbins et al. 2009; Armitage et al. 2011). Other species show no sex-bias in dispersal propensity but only in dispersal distances (Tucker et al. 1998; Loew 1999), depicting the complexity of the whole phenomenon. Therefore, despite the strong canalizing effect of the mating system towards a certain pattern of SBD, the trade-offs between costs and benefits of dispersal or philopatry determine whether and in which direction a sex-bias in dispersal tendencies and/or distances develops. As a result, the current general 
agreement is that the evolution of SBD was driven by a complex interaction of multiple selection pressures, but no consensus exists about the relative importance of each factor (Ronce et al. 2001). Asymmetries introduced by mating system on patterns of local competition interact with local competition for resources and mates, inbreeding avoidance, kin-cooperation, dispersal tendencies and tenure length of the opposite sex, sex-specific mortality and phylogeny (Greenwood 1980; Dobson 1982; Waser et al. 1986; Pusey and Packer 1987; Schwartz et al. 1998; Perrin and Mazalov 2000; Van Noordwijk and Van Schaik 2001; Lehmann and Perrin 2003; Bowler and Benton 2005; Nunes 2007; Clutton-Brock and Lukas 2012). Feedback loops between SBD and these factors would then maintain and possibly pronounce SBD tendencies.

However, disentangling the evolution of SBD remains difficult because of methodical and conceptual problems. First, definitions of dispersal vary between studies, building either on the change of the social environment or the locality (Clutton-Brock and Lukas 2012). Consequently, strategies and methods to quantify SBD differ, making even comparisons of different studies on the same species problematic (Le Galliard et al. 2012). Second, most studies do not go beyond reporting the sex-bias, which makes comparisons between species difficult (Roy et al. 2012). Third, empirical studies usually fail to achieve a clear discrimination between evolutionary causes, because SBD can evolve as an answer to different selective pressures (Ronce et al. 2001). Finally, distinguishing ultimate factors of dispersal from factors acting on the proximate level (see below) proves to be difficult in many cases (Lidicker and Stenseth 1992). While ultimate factors describe the selective pressures that caused the existence (or absence) of SBD in recent species, proximate mechanisms are those factors that trigger the emigration of an individual. Since proximate dispersal mechanisms are the result of evolutionary processes, they have been used as indirect evidence for the ultimate causes of dispersal (Lidicker and Stenseth 1992; Alberts and Altmann 1995a). However, because of the close link some studies fail to make the distinction, and ultimate explanations are used to explain behaviour on the proximate level (e.g. Armitage et al. 2011). The limited knowledge about which ultimate cause should influence which proximate mechanism calls for a careful consideration of observed dispersal behaviour. A good characterization of the dispersal behaviour on the proximate level and carefully assessing the consequences of dispersal for the competitive regimes (resources and mates) and inbreeding risk will be 
necessary to gain a better understanding about why SBD evolved and which mechanisms translate SBD propensities into individual behaviour.

\section{INBREEDING AVOIDANCE - AN IMPORTANT FACTOR FOR SEX-BIASED DISPERSAL?}

Among the different ultimate factors, inbreeding avoidance is one of the most often quoted but also most debated mechanisms for the explanation for SBD patterns (Greenwood 1980; Dobson 1982; Moore and Ali 1984; 1985; Waser et al. 1986; Pusey 1987; Pusey and Packer 1987; Pusey 1988; Clutton-Brock 1989; Wolff 1994; Lukas and Clutton-Brock 2011; CluttonBrock and Lukas 2012). Inbreeding avoidance has been regarded as an important driving force of SBD because of the strong potential effectiveness of dispersal to prevent consanguineous matings and, thus, the occurrence of inbreeding depression by spatially separating relatives (Charlesworth and Charlesworth 1987; Pusey and Wolf 1996; Charlesworth and Charlesworth 1999; Charlesworth and Willis 2009). Breeding between relatives is supposed to have a negative impact on fitness, because it leads to a reduction of heterozygosity and potentially to the unmasking of deleterious genetic variants. However, when inbreeding avoidance was first proposed as driving SBD, researchers were at first confronted with a conundrum. Since females invest more than males in reproduction, they should also face higher costs when producing inbred offspring. As a result, they should be choosier when selecting mates, and, therefore, in contrast to empirical evidence, disperse more readily (Trivers 1972; Greenwood 1980; Waser et al. 1986). A solution to this apparent paradox brought the realization that nepotism effects (the possibility to profit from the presence of relatives) can favour female philopatry (Moore 1992). Female reluctance to disperse and mate choice in favour of unrelated males would boost male dispersal (Lehmann and Perrin 2003). Hence, the importance of female choice in different (theoretical) scenarios varied and in the majority of cases evolutionary stable strategies evolved based on a combination of inbreeding avoidance with other factors (Perrin and Mazalov 1999; Perrin and Mazalov 2000; Perrin and Goudet 2001; Lehmann and Perrin 2003). Notably, in these models a strong sex-bias in dispersal tendencies was the most stable strategy, if local competition was removed and inbreeding was the only ultimate factor involved, but the direction of the bias was random.

Still, isolating the role of inbreeding avoidance in the evolution of SBD remains difficult. The difficulties may partly arise because for many species with SBD past 
evolutionary pressures are not detectable anymore. Dispersal distances have been used as a measure of whether SBD is a consequence of inbreeding avoidance (Greenwood 1980; Waser 1985; Keane 1990; Lambin et al. 2001; Lawson Handley and Perrin 2007). But these distances not always seem to be adapted to avoid inbreeding, and some studies failed to detect negative impacts of inbreeding despite relatively high frequencies of occurrence (Duarte et al. 2003). Another problem is that inbreeding avoidance seems to be hard to distinguish from proximate mechanisms and therefore is often used as an argument on both levels (Lidicker and Stenseth 1992; Armitage et al. 2011). A possible way to tackle these problems could be to start considering under which conditions dispersal propensities remain rather flexible and need proximate, situation-dependent cueing and under which conditions they become a characteristic of one sex (Long et al. 2008). For example, differences in the rigidness of SBD tendencies should indicate differences in canalizing selective pressures (Lidicker and Stenseth 1992; Herrera et al. 2011). Theoretically, if inbreeding avoidance was an important agent in the evolution of SBD, species with strong SBD rates should have a low potential for inbreeding, especially if no secondary dispersal occurs. Assessing the consequences of SBD for the existence or absence of inbreeding risk could help us understand the effectiveness of SBD for avoiding inbreeding. This thesis will investigate whether this assumption is valid for a small promiscuous primate with male-biased dispersal, for which secondary dispersal is a rare event.

\section{DISPERSAL CUES AND TRIGGER - WHO GOES WHEN, WHERE AND WHY?}

Proximate mechanisms of dispersal are the result of the selective pressure exerted by ultimate motivations for dispersal (Long et al. 2008). These cues comprise a versatile number of internal and external mechanisms stressing again the complex nature of dispersal. Present conceptual approaches assume that dispersal decisions are based on highly individualized combinations of cues allowing for individual flexibility during dispersal (Bowler and Benton 2005; Clobert et al. 2009; Bonte et al. 2012), and therefore stress the need for an individualbased perspective, where dispersal is considered as a multi-phase life-history process. The phases comprise the three commonly differentiated phases of the dispersal process (Andreassen et al. 2002; Ronce 2007; Clobert et al. 2009): (1) emigration (departure), (2) transfer (a vagrant stage), and (c) immigration (finalization of the movement phase, settling). Individual condition-dependent dispersal strategies seem to be common among species, 
probably because they offer the advantage over unconditional strategies that individuals are able to match their own internal, motivational state to physical and social conditions (Danchin et al. 2001; Ims and Hjermann 2001; Massot et al. 2002; Bonte and De La Peña 2009; Clobert et al. 2009).

Most information on proximate cues are available for the emigration and immigration phase of dispersal. Proximate triggers during these phases include individual attributes like genetic or hormonal priming (Holekamp 1986; Trefilov et al. 2000; Dufty and Belthoff 2001; Dufty et al. 2002; Krackow 2003; Pasinelli et al. 2004; Haag et al. 2005), body condition (Nunes and Holekamp 1996; Nunes et al. 1998), and personality (Dingemanse et al. 2003; Hoset et al. 2011; Cote et al. 2012) as well as external factors like parental or conspecific aggression (Van Vuren and Armitage 1994; Isbell and Van Vuren 1996; Smale et al. 1997), social environment and stability (Alberts and Altmann 1995a; Long et al. 2008; Jack et al. 2011), population density (Matthysen 2005; Roy et al. 2012) and habitat conditions including interspecific interactions (Ims and Hjermann 2001). Their relative importance and interactions vary among species depending on the sensitivity of a species towards these cues (on the physiological and cognitive level). Cues are supposed to affect an individual by changing its internal or motivational state (Clobert et al. 2009).

Information on cues acting on the transfer phase is more limited. The most important aspects during the transfer are those that affect the choice of settlement area or group. Mechanisms of habitat choice include body condition (Stamps 2006), habitat quality (Wauters et al. 1995; Matthysen and Currie 1996), habitat imprinting (Burns 2005; Mabry and Stamps 2008a; Mabry and Stamps 2008b), conspecific attraction (Reed and Dobson 1993; Stamps 2001), public information like the information conspecifics convey through their condition (Danchin et al. 2001) and population densities (Matthysen 2005). Positive as well as negative density-dependent immigration patterns have been observed (Matthysen and Currie 1996; Ims and Yoccoz 1997; Fisher et al. 2009). Important aspect during the transfer is also the range of perception of an animal, because it determines the efficiency of different exploration strategies and dispersal movements (Zollner and Lima 1999; Mech and Zollner 2002; Zollner and Lima 2006).

Based on this information, this thesis aims at adding to current knowledge of condition-dependent strategies by identifying the basic proximate mechanisms that are involved in the activation of the dispersal process. This information on basic mechanisms will 
be crucial to evaluate whether and how the behaviour of individuals can be used to identify ultimate aspects of dispersal.

\section{DISPERSAL MOVEMENTS - MISSING PIECES OF A PUZZLE}

The transfer stage is usually assumed to be the dispersal phase that poses the greatest threats to the survival of an individual, because of an increased risk of predation and starvation. However, the picture remains incomplete because crucial information on how dispersers actually move is usually lacking. Collecting data on the movement pathways of dispersers is extremely difficult, because for many species the event can be based on an instantaneous decision, without any obvious prior behavioural signs from the animal itself or its conspecifics. Relocation strategies can be very different, ranging from gradual shifts (Jones 1989) to distinct relocations of ranging areas (Bearder 1987; Mech 1987; Holekamp and Sherman 1989). Besides the form, the duration of the transient stage apparently also varies remarkably among species, with very short settling periods of single individuals or several parallel dispersing individuals to prolonged periods leading to single floaters or the formation of single sex bands (Holekamp 1986; Rajpurohit and Sommer 1993; Smale et al. 1997; Fernandez-Duque and Huntington 2002; Roper et al. 2003; Schoof et al. 2009). Dispersal strategies can also differ within a species, leading to different types of emigrants or distinct dispersal syndromes for colonization (Roff and Fairbairn 2001; Duckworth and Badyaev 2007; Clobert et al. 2009; Le Galliard et al. 2012). Finally, the shape of dispersal kernels (the density function of dispersal distances) remains unknown for most species, though this information constitutes an important aspect of dispersal capacities (Koenig et al. 1996; Le Galliard et al. 2012). A problem associated with the study of dispersal distances is the fact that short distance dispersal events are often easier to detect than long distance dispersal. Factors influencing dispersal distances can be genetic elements (Bitume et al. 2010; Selonen and Hanski 2010), population density and social factors (Pasinelli et al. 2004).

Due to the limited knowledge, the predictive framework, what form dispersal movements should take under which conditions, remains limited to a few tentative working hypotheses (Van Dyck and Baguette 2005). Van Dyck and Baguette (2005) differentiated in their work between normal (foraging) and specialized movement through which dispersal can be achieved and characterized differences between the two strategies. Currently, most efforts of studying movement strategies during dispersal are of theoretical nature, using 
modelling approaches to study the possible advantages and disadvantages of different movement strategies (Zollner and Lima 1999; Conradt et al. 2003; Heinz and Strand 2006). However, only increasing our knowledge about dispersal capacities will allow for an evaluation of the significance of theoretical approaches and help understand the costs of dispersal for an individual. One useful method to study movement strategies in unfamiliar areas are translocation experiments, where animals are relocated over different distances from their ranging area. This would provide information about navigation capabilities and the extent of spatial knowledge that are available to a disperser (Zollner and Lima 1999; Mech and Zollner 2002; Zollner and Lima 2006).

In fact, evidence is accumulating that in many cases dispersal movements show nonrandom patterns. However, to which degree dispersers are able to make informed decisions during the dispersal process is still unclear (Delibes et al. 2001; Conradt et al. 2003; Conradt and Roper 2006; Clobert et al. 2009). One indication that dispersers make informed decisions during dispersal comes from the fact that animals would need to produce much larger numbers of offspring, if dispersal was random as it is in plants (Ims and Yoccoz 1997). Another explanation could be that habitat matrices are less dichotomous concerning the suitability but represent a graded matrix, facilitating the successful dispersal of individuals. Finally, the particular lack of information on some behavioural aspects of this process also impedes the possibilities to determine the degree of flexibility that could be intrinsic to this phase. Possibly, similar to condition-dependent dispersal, the form of transfer movements can help to reduce costs of dispersal. Hence, detailed empirical insights into dispersal movements are the missing pieces in the puzzle of a phenomenon that has otherwise received enormous attention. Therefore, one main focus of this thesis is on these behavioural aspects of dispersal, to provide an impulse for future research and theoretical considerations.

\section{SEX-SPECIFIC DISPERSAL PROPENSITIES AND THEIR CONSEQUENCES IN GREY MOUSE LEMURS (MICROCEBUS MURINUS)}

This thesis investigates the dispersal process and possible consequences for one of the beststudied species of Malagasy lemurs, the grey mouse lemur (Microcebus murinus, J.F. Miller, 1777). They are small $(60 \mathrm{~g})$, omnivorous primates, and can be found in most remaining forests in southern and western Madagascar (Rasoloarison et al. 2000; Kappeler and 
Rasoloarison 2003; Mittermeier et al. 2010). Distribution of populations within continuous habitats can be patchy and densities can vary by a factor of 10 (Kappeler and Rasoloarison 2003; Fredsted et al. 2004). Grey mouse lemurs spend their activity period mainly solitarily, but individual home ranges of about 1.5ha overlap extensively between and within sexes. Information on dispersal trends in grey mouse lemurs are mainly based on previous population genetic and capture-mark-recapture (CMR) studies (Wimmer et al. 2002; Radespiel et al. 2003; Eberle and Kappeler 2004a; b; Fredsted et al. 2004; Fredsted et al. 2005; Eberle and Kappeler 2006; Gligor et al. 2009). Grey mouse lemurs correspond to the typical pattern of mammals with strongly male-biased dispersal both in frequencies and distances. This pattern is consistent among study areas. Some studies indicate that females benefit from philopatry in the form of increased survival for themselves and their offspring (Eberle and Kappeler 2006; Lutermann et al. 2006). For a primate, grey mouse lemurs have a rather fast life history. They are completely self-sufficient at an age of about two to three months and can reproduce at an age of about nine months (Eberle and Kappeler 2006, Huchard et al. 2011). With their additional broad distribution in Madagascar and the modal mammalian dispersal pattern, they represent an interesting model for studying fundamental aspects of dispersal behaviour.

However, despite some information on dispersal trends and dispersal distances inferred from genetic and CMR studies, knowledge about the behavioural processes that lead to this distribution of individuals remain scarce. Since dispersal rates are strongly malebiased, parentage analyses commonly fail to assign subadult males to parents (up to $80 \%$ ) (Radespiel et al. 2003), hampering our understanding of dispersal distances and the dynamics of individual movements among population patches. Data from CMR studies can usually not differentiate between mortality and dispersal of males (Kraus et al. 2008). This thesis aims at expanding not only our understanding of SBD in grey mouse lemurs, but also our knowledge about dispersal in general, by addressing proximate aspects of the dispersal process and depicting detailed information of dispersal movements, duration and timing (Chapter 1). Through behavioural observations and telemetry, I determined movement styles, dispersal distances and the duration of the transfer phase, improving our understanding of dispersal capacities and potential costs of dispersal. Additionally, a translocation experiment provided a rare opportunity to study movements in unfamiliar areas and possible constraints on them. 
In Chapter 2, I evaluate the basic proximate factors that promote (or prevent) dispersal, by using capture-mark-recapture and parentage data collected between the years 1994 and 2010. I evaluated whether body mass could predict the probability of a male still being a resident or whether it had already dispersed. Moreover, I looked at the relationship of body mass to travelled dispersal distances.

Dispersal decisions are assumed to be preceded by changes of the internal or motivational state of individuals (Le Galliard et al. 2012). Hormones seem to play a crucial role in behavioural changes and the role of hormones like corticosterone in stimulating dispersal activity has been documented for a few species (Belthoff and Dufty 1998; Dufty and Belthoff 2001; Dufty et al. 2002; Meylan et al. 2002). But for a large majority of animals, collection of adequate samples is often difficult and/or corresponding hormone essays are not (yet) existing. However, indirect information about differences in the motivational state of individuals can be inferred from the sequence of behavioural processes. In Chapter 3, I exemplify this approach by modelling the feeding behaviour of subadult males and females using mixed hidden Markov models (HMM).

Given male skew in dispersal rates and the singularity of natal dispersal in a grey mouse lemurs' life, the question arises whether inbreeding can be effectively avoided under these conditions. In Chapter 4, I therefore combined trapping and parentage data to evaluate the risk of inbreeding, mechanisms of inbreeding avoidance and possible fitness advantages of high individual genetic diversity.

Finally, I summarise the major results of the thesis, discuss their implications for the study of SBD in relation to findings from other studies, and provide an outlook on future research needed to complement our understanding of SBD. In combination, this thesis will supplement already existing knowledge about dispersal for grey mouse lemurs and annex proximate aspects of dispersal with consequences of dispersal on the ultimate level to answer the questions, what causes individuals to disperse and what are the consequences from an evolutionary perspective. 
Chapter 1: Dispersal Movements

\section{Walk the line - Dispersal movements of gray mouse lemurs}

with Manfred Eberle and Peter M. Kappeler

in Behavioral Ecology and Sociobiology (2012): in press 


\section{ABSTRACT}

Despite the importance of dispersal for individuals and populations, little is known about the actual dispersal process in most species. We observed 90 subadult gray mouse lemurs small, arboreal, nocturnal primates - in Kirindy Forest in western Madagascar, to determine the behavioral processes underlying natal dispersal. Twelve radio-collared males dispersed over distances between 180 and $960 \mathrm{mF}$ (1-7 home range diameters) away from their presumed natal ranges. Dispersal forays were fast and highly directed, and thus distinct from routine movements. Contrary to expectations of current hypotheses on potential differences between different types of dispersal movements, their special movement style did not prevent dispersers from interrupting forays to exploit resources they encountered during their forays. Data from a translocation experiment indicated that highly directed dispersal or search forays reflect a general strategy for large-scale exploration away from familiar sites in this species. A prolonged transfer phase was also observed, with regular commuting between old and new sites for up to 14 days, which probably served to moderate costs of unfamiliarity with a new site. In conclusion, the dispersal process of gray mouse lemurs is characterized by high intra- and interindividual consistency in movement strategies, but variation in the duration of the transfer phase. The observed dispersal movement style represents an effective strategy balancing costs of dispersal with the need to gather an appropriate level of information about potential dispersal target sites.

Keywords: natal dispersal, dispersal movements, transfer, translocation, Microcebus murinus

\section{INTROCUCTION}

Dispersal is a key process in individual life histories and a central topic in ecology, evolution, and conservation because it affects the fitness of individuals, determines their distribution, and has important consequences for the demographic and genetic structure of populations (Clobert et al. 2004; Nunes 2007). Dispersal is defined as a movement (active or passive) of an organism or a propagule from its site or group of origin to its first or subsequent breeding site or group (Shields 1987; Lidicker and Stenseth 1992; Clobert et al. 2009). Many animals show a sex-bias in dispersal rates, with males being the predominantly dispersing sex in mammals (Greenwood 1980). In the past years, much effort has been put into studying the 
influence of inbreeding avoidance and competition for resources and mates on the evolution of sex-biased dispersal, the associated costs and benefits of dispersal for individuals (but also populations), and how they vary in space and time (Greenwood 1980; Dobson 1982; Waser et al. 1986; Pusey and Packer 1987; Smith 1987; Alberts and Altmann 1995a; Isbell and Van Vuren 1996; Dufty and Belthoff 2001; Andreassen et al. 2002; Pasinelli et al. 2004; Yoder et al. 2004; Boinski et al. 2005; Bowler and Benton 2005; Nunes 2007; Ronce 2007; Bonte et al. 2012; Clutton-Brock and Lukas 2012).

However, our understanding of dispersal is limited because for many species we lack detailed information on the behavioral processes underlying different phases of dispersal (emigration, transfer, and immigration). Because of the practical difficulties of observing this often once-in-a-lifetime event, studying the transfer phase of dispersal remains especially difficult. Systematic analyses of the possible advantages and disadvantages of different transfer strategies are therefore mainly of a theoretical nature because they rely on modeling of the transfer phase of dispersal under different assumptions and conditions, mainly in a patchy landscape matrix. For instance, some of these studies investigated the effectiveness of different movement strategies (random and systematic searches), or they explored which type of movement develops under a given set of conditions (Zollner and Lima 1999; Wiens 2001; Conradt et al. 2003; Heinz and Strand 2006; Barton et al. 2009). However, there is a gap between modeling approaches and empirical studies in that the number and sophistication of theoretical models of dispersal far exceeds our knowledge of actual animal movements. First approaches to generate working hypotheses to evaluate empirical dispersal movements differentiate between dispersal via routine movements or special movements (Van Dyck and Baguette 2005). If dispersal is accomplished by special movements, it is proposed that the movements should differ from routine movements, in general features like spatial scale of displacement, speed of movements, configuration of trajectory, and responses to conspecifics and resources during movement.

In the present study, we investigated dispersal behavior and movements of the nocturnal, solitary gray mouse lemur (Microcebus murinus, J.F. Miller, 1777) in the Fôret de Kirindy, a dry deciduous forest in western Madagascar (Eberle and Kappeler 2004a; b). Gray mouse lemurs are small (60g), omnivorous primates and can be found in most remaining forests in southern and western Madagascar (Mittermeier et al. 2010). Despite their solitary activity, individual home ranges of about 1.5 ha overlap extensively between and within 
sexes. This fact distinguishes gray mouse lemurs from most other species for which data on the dispersal process are available (Bearder 1987; Mech 1987; Steen 1994; Estes-Zumpf and Rachlow 2009). During the day, closely related females form stable sleeping groups, whereas adult males only occasionally share sleeping sites. For the duration of the short annual mating season, males roam widely, more than quadrupling their habitual home range. After 2 months of gestation, females give birth to one to four young, which are weaned at the age of about 2-3 months (Schmid 1998; Schmid and Kappeler 1998; Fietz 1999; Radespiel 2000; Eberle and Kappeler 2002; 2004a; b).

Previous population genetic studies revealed that dispersal in gray mouse lemurs corresponds to the general mammalian trend of male-biased natal dispersal (Eberle and Kappeler 2002; Radespiel et al. 2003; Eberle and Kappeler 2004a; b; Fredsted et al. 2004; Fredsted et al. 2005). With the onset of the austral fall, subadult males start to disperse (Eberle and Kappeler 2004a; Kraus et al. 2008). However, the actual behavioral processes generating this distribution of individuals remain unknown. Therefore, the main focus of our study was to investigate how gray mouse lemur males disperse and to describe different aspects of the dispersal process. If dispersal is achieved through a particular type of movement, potential differences to routine movements include a high degree of linearity of movement pathways, high movement speed, and no responses to resources or conspecifics (Van Dyck and Baguette 2005). Below, we portray (high resolution) dispersal movements and contrast them with normal movements as well as roaming movements during the mating season, to investigate whether dispersing gray mouse lemurs adopt a behaviorally different movement style during dispersal. We also present results of a translocation experiment whose aim was to explore how gray mouse lemurs move in unfamiliar habitats and whether they were able to successfully home back to their usual home range. Finally, to further test whether gray mouse lemurs alter their movement behavior during dispersal we compared travel distances of mouse lemur males during different situations (routine, dispersal, roaming, and translocation).

\section{METHODS}

\subsection{Study site and capture}

The study was conducted within a 12,500-ha forestry concession of the Centre National de Formation, d'Etude et de Recherche en Environnement et Foresterie (CNFEREF) de 
Morondava in Kirindy Forest. This dry deciduous forest is situated $60 \mathrm{~km}$ northeast of Morondava in western Madagascar $\left(44^{\circ} 39^{\prime} \mathrm{E}, 20^{\circ} 03^{\prime} \mathrm{S}\right)$. The region is characterized by pronounced seasonality with a single rainy season between December and March and a dry season from April to November (Kappeler and Fichtel 2012a). The study took place in a 60-ha area, locally known as CS7, containing a rectangular system of small foot trails at 25- to 50-m intervals (described in Eberle and Kappeler 2004a). For the translocation experiment, data were additionally collected within a second grid system, locally known as CS5 (26ha, described in Lührs et al. 2009).

\subsection{Data collection and processing of dispersal, normal, and roaming movements}

Gray mouse lemurs in CS7 have been continuously captured, marked, and studied since 1994. In order to collect behavioral data for this study, we captured subadult individuals and supplied them with radio collars (Holohil Systems Ltd., BD-2C, 1.8g). Trapping procedures and animal handling followed the protocol described in Eberle and Kappeler (2004a). Individuals were classified as subadult by their small body mass $(<55 \mathrm{~g})$, small size, and the absence of a subdermal passive transponder. Trappings were conducted on three consecutive nights once per month in a central capture area (9ha, 180 traps) and twice per year in the surrounding areas (25ha, 210 traps). Altogether, we equipped 90 subadult individuals with radio collars, 28 females and 62 males. We detected no obvious signs of adverse effects of the radio collars on individuals. At the end of the study, an attempt was made to remove all radio collars. To this end, we conducted special, targeted trapping sessions for dispersers which had left the study area. Twelve individuals (two females, ten males) were not recaptured at the end of the study. One female was only recaptured a year after this study, and she showed no signs of adverse effects caused by the radio collar.

Data of dispersal movements were collected during the dry seasons between March and June 2007, March and May 2008, April and September 2009 and April and September 2010. Between 1800 and 2400 hours, we determined locations of radio-collared animals between one and three times per night. Data points were considered to be statistically independent of each other if they were collected at least 20min apart (Dammhahn and Kappeler 2009). If individuals were sighted, their exact position was determined with reference to the trail system or with the help of a GPS device (Garmin GPSMAP 76CSx, accuracy of position $<10 \mathrm{~m}$ RMS). When individuals were not visible, their location was 
determined via triangulation to the nearest $25 \mathrm{~m}$. Spatial data were recorded as UTM coordinates and processed and visualized in ArcGIS 9.3 (Esri) and the toolbar "Home Range Tools" (HRT) for ArcGIS ${ }^{\circledR}$ (Rodgers et al. 2007). If an animal moved outside its regular home range, we tracked this particular individual continuously in order to determine direction and distance of movements in detail.

Number of independent data points for calculation of regular home ranges and the time period during which they were collected varied between individuals because of predation events, variation in the life span of radio collars, length of field season, and dispersal events. As a result, these spatial data were collected over periods ranging from 1 week up to 7 months. Dammhahn and Kappeler (2009) determined that 50 independent locations are sufficient to calculate representative minimum convex polygons (MCP) of gray mouse lemur's home ranges. If we had more data points per individual, we reduced them to a random sample of 50 points, to balance sample size among individuals. Control calculations of home range location using all available data for individuals with more than 50 data points revealed stability of all home range positions independent of sampling period.

\subsection{Data collection and processing of movements of translocated individuals}

Translocated individuals were captured during additional, targeted capture sessions in smaller areas, using about 40 traps. These animals were trapped twice within 5 days, once to translocate them from their familiar range for the experiment and again to return them to their familiar area. We weighed them before and after the experiment to check their health status. Following the experiment, we kept mouse lemurs for one night at the research station and supplied them with bananas and raisins.

Six males were translocated for a period of 3 days between August and September 2010. Translocation distances ranged from 0.2 to $2 \mathrm{~km}$, equaling 1 to 14 home range diameters. All six translocated individuals originated from the grid system CS7, and except for one individual, all were translocated within CS7. For these five individuals, we knew their natal home range, so we could ensure that we could transfer them to an unfamiliar area within the same grid system. They were translocated over 200 and $600 \mathrm{~m}$ (about one to three home range diameters). The remaining individual was transferred to another grid system (CS5) $2 \mathrm{~km}$ away because we did not know its natal origin. Because $2 \mathrm{~km}$ are well above the 
maximal observed dispersal distance, this strategy ensured that we translocated this individual to an unfamiliar area where we could also observe it more easily.

To release the animals, we positioned traps with the trap door closed on branches at about $1.5 \mathrm{~m}$ height at around $1745 \mathrm{~h}$. At $1800 \mathrm{~h}$, we opened the trap door. From that moment, we recorded every minute the whereabouts of the animal and their behaviors (e.g., feeding, interactions, etc.) via one-zero sampling (Martin and Bateson 1993). The animals decided on their own account when they would leave the trap (5- to 60-min latency). Every animal was released at a different position. We observed one individual at a time and followed it over the whole activity period from dusk till dawn for the three nights, yielding between 1,380 and 1,815 location points per individual (median=1,784) recorded during focal observations. Contact time during observations varied between $68 \%$ and $84 \%$ (median=82\%) because the focal animals were occasionally out of sight. On the fourth evening, animals were retrapped (see above) to return them to their habitual home range.

We calculated the size of prevailing ranges (area used by translocated individuals) as 95\% kernels for each night separately, using independent locational data points (20-min intervals) from the period when an individual was active. The size of these areas was compared using Friedman's test of the "stats" package (C) R Core Team and contributors worldwide). Pairwise comparisons between nights were conducted using a paired Wilcoxon rank sum test, and $\alpha$ levels were adjusted for multiple testing using the Bonferroni correction (Rice 1990). Results of statistical tests and a figure of prevailing range sizes were generated with the software R vers. 2.14.2 (@ The R Foundation of Statistical Computing).

\subsection{Average travel distances per hour}

To test whether sex and different circumstances (routine, dispersal, roaming, and translocation) affect movement behavior, we compared average travel distances. Data on routine movements within the home range were based on focal observation of 19 subadult females (no. of observations, 1-27) and 36 subadult males (no. of observations, 3-22) collected between 18 and 23h during March and May 2008, April and September 2009, and April and September 2010. Four animals per night in changing order and combinations were observed for 40-min intervals. Data on roaming movements originated from focal observations of 19 males (no. of observations, 1-16) from October to November 1999 to 2001. One to nine males per night were observed (50-60 $\mathrm{min}$ ) by one to three observers. 
Travel velocities during dispersal and translocation were calculated for six individuals. Mean standardized hourly travel distances per individual were computed. To do so, we calculated the average distance moved per minute during observation bouts and extrapolated these distances up to hourly distances. We compared travel distances during routine movements of subadult males and females using an unpaired Wilcoxon rank-sum test to determine whether males differ from females in general. To test for equality of male travel distances in different situations (routine, dispersal, roaming, translocation), we compared them separately using Kruskal-Wallis rank sum-test and Nemenyi-Damico-Wolfe-Dunn test, also known as Dunn's post hoc test (Hollander and Wolfe 1999). For Dunn's post hoc test, exact $p$ values and $99 \%$ confidence interval were approximated via Monte Carlo resampling based on 90,000 permutations. Graphics were generated and statistical tests computed with the packages "coin" (Hothorn et al. 2008b) and "multcomp" (Hothorn et al. 2008a) in R vers. 2.14.2 (C The R Foundation of Statistical Computing).

\section{RESULTS}

\subsection{Dispersal movements}

Dispersal movements differed from "routine" movements. Dispersal was characterized by a distinct displacement of the home range, which was accomplished through highly directed movements (Fig. 1). None of the 28 females ever made attempts to relocate their home range, but for 12 out of 62 males, we were able to collect data on details of the dispersal process (duration of the transfer phase, distance). For the remaining 50 males, which did not shift their home range, 19 died due to predation. However, parentage analyses suggested that most of these 50 males were immigrants when they were captured the first time (unpublished data). These population-wide parentage analyses were highly effective for females, assigning about $80 \%$ of them to mothers, but failed to detect mothers for $75 \%$ of the males.

Ten of the 12 males dispersed "successfully" in the sense that they relocated their home range to a new site. We never observed them prospecting another site afterwards, suggesting that secondary dispersal is rare in this species. These males dispersed over distances ranging from $180 \mathrm{~m}$ to $960 \mathrm{~m}$ between April and June, except for two males, which dispersed during the mating season in October/November (220- and 350-m dispersal distance, respectively). The two unsuccessful dispersers remained in their natal area after 


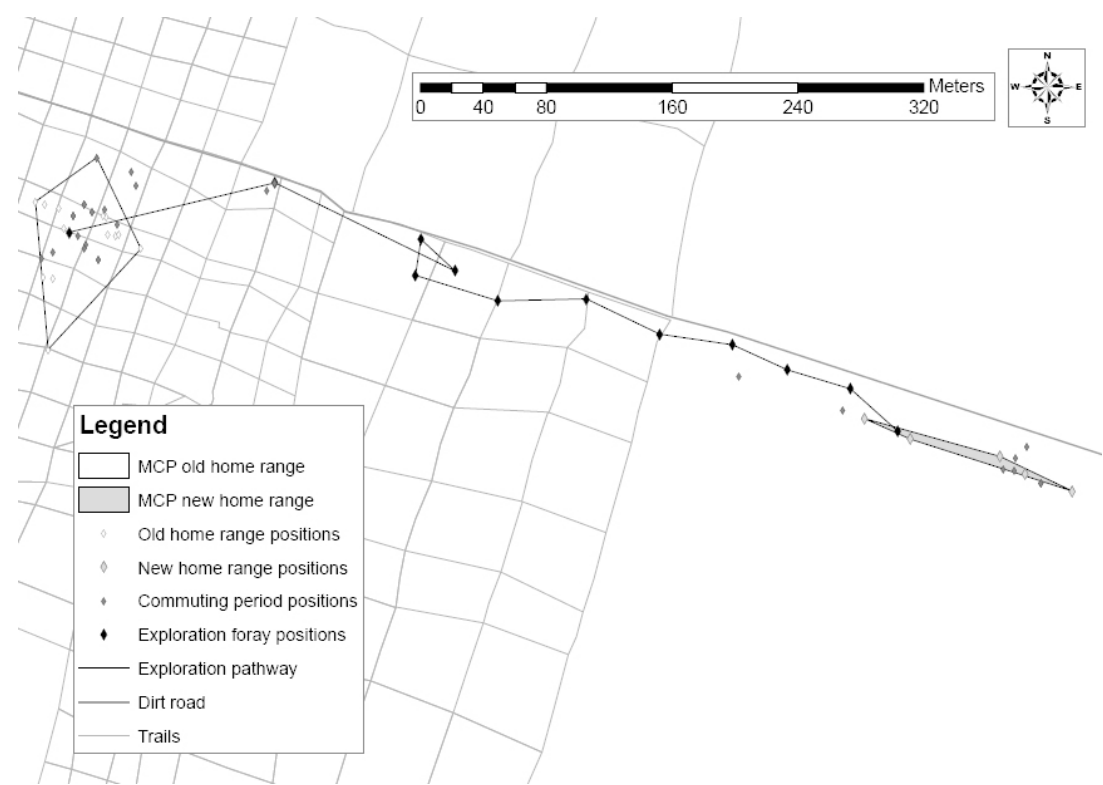

FIG. 1: FIRST EXPLORATION FORAY AND COMMUTING OF SUCCESSFUL DISPERSER 22F7: AN EXAMPLE OF A TYPICAL DISPERSAL EVENT

The figure shows a section of the grid system. Different symbols indicate different phases of the dispersal process. The black line indicates the pathway during the first exploration foray. The 95\%-MCPs for old and new home range were calculated based on statistically independent locations. We used 50 randomly chosen data points (white diamonds) for the old home range, but had only five locations for the new home range (big, light gray diamonds). Positions taken during the commuting period (small, dark gray diamonds) were collected on different days and thus depict no movement path. They are shown here to illustrate the fidelity of the disperser to its dispersal foray during the commuting phase. These positions were not included into MCP calculations.

initial dispersal trials in June and August. Their forays away from their natal home range had a distance of $100 \mathrm{~m}$ and $280 \mathrm{~m}$ (Fig. A1, A2, A for Appendix). Individuals showed no obvious behavioral signs of imminent departure and did not seem to face increased aggression prior to and during dispersal. How dispersers chose where to go and where to stay remains unclear. There were no obvious landscape features that might have guided or constrained dispersal direction in this continuous forest. However, dispersers seemed to choose to disperse more frequently towards the eastern and northeastern part of our study site (2 out of 12; Fig. 2). Unfortunately, we lack data on habitat structure and population densities for the settling areas, so that the actual mechanisms of habitat selection remain unknown. Transect captures and sightings of conspecifics during the night indicate that all dispersers transferred through and to areas inhabited by other gray mouse lemurs. Another indication that conspecific presence might be important comes from observations of one successful disperser that dispersed in the direction of a natural border of our study population in the northwestern part of our study area, where population density decreased markedly (Fig. 2). 


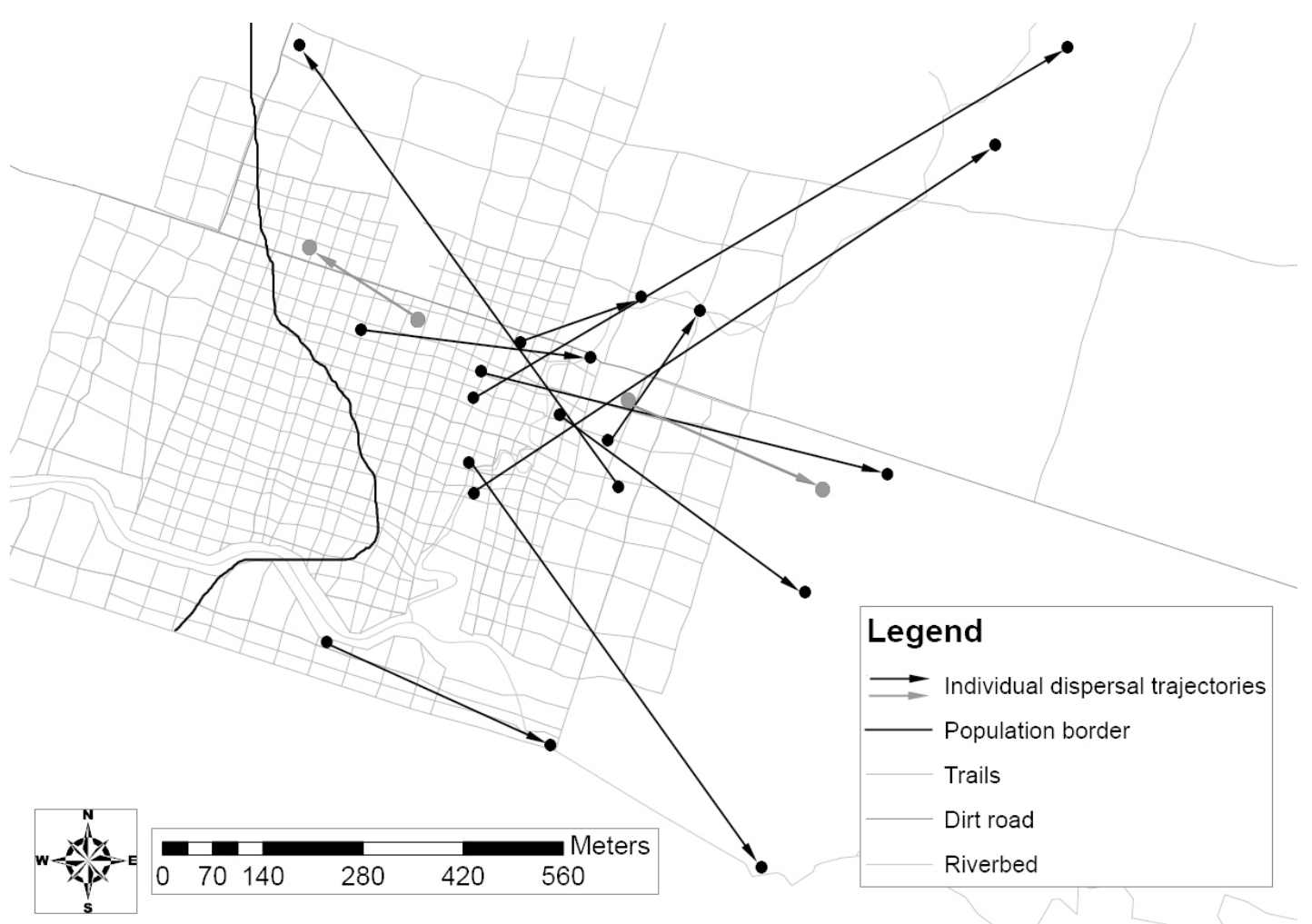

FIG. 2: DIRECTIONS AND DISTANCES OF INDIVIDUAL DISPERSAL TRAJECTORIES Illustrated are the directions and distances of dispersal for $n=12$ male dispersal events (dispersal trajectories of successful dispersers are depicted in black, unsuccessful dispersers are depicted in gray). Arrows indicate the direction of dispersal and point from the central position of the old home range of a given individual towards the new home range. The black line sketches the natural border of our population, where to the western side population density strongly decreases. For the other directions the total extent of the population remains unknown so far.

On the night of dispersal, this male moved beyond that edge, but we do not know the full extent of its foray because the transmitter signal was lost. The next morning, he had returned to the periphery of the study population, where he subsequently established his home range.

The duration of the transfer phase varied between individuals (range, 1-14 nights), but all observed dispersal distances could be covered within one night. Four successful dispersers completed dispersal within one night (distance between old and new home ranges, $320 \mathrm{~m}$ and $830 \mathrm{~m}$ ). They exhibited one directed movement towards their new home range and never returned to their old home range. For six individuals (four successful, two unsuccessful dispersers), the process lasted between 7 and 14 nights. These animals moved back and forth between their old and new home range, sometimes even within the same night ("dispersal forays", see below). For the remaining two individuals which dispersed 
during the mating season, we lack information on the duration of the transfer phase and could only establish that they had relocated their home range after the end of the mating season.

For 6 of the 12 males (four successful, two unsuccessful dispersers), we obtained additional detailed information on dispersal movements (Fig. 1, A1, A2). Dispersal forays were highly directed movements away from the natal area and differed strongly from roaming and routine movements within the home range (Fig $A 3, A 4)$, which usually do not exhibit such a high degree of linearity. In cases where the transfer phase lasted longer than one night, dispersal forays occurred always in the direction where successful dispersers would ultimately establish their new home range (Fig. 1). Like successful dispersers, unsuccessful dispersers also never changed the destination of their dispersal forays to investigate other areas (Fig. A1, A2). Commuting between the old and new home range could occur on a daily basis. During the transfer phase, an individual might also leave for several nights and then return to its old home range for several nights, having sleeping sites within both areas (Fig. 1, A2). Movements back to the natal range were also highly directed (Fig. A2). Remarkably, we observed these individuals to move considerable distances (about $10 \mathrm{~m}$ ) continuously on the ground at high speed, something we never observed during routine movements. However, if animals encountered food resources (insects, gum) during forays, they did not forego their exploitation (Fig. A2).

\subsection{Translocation movements}

Translocated individuals did not walk randomly in space, but established "prevailing ranges". These prevailing ranges increased gradually in size during the first night of translocation and again during the following two nights (Friedman rank-sum test: $\chi 2=6.33, d f=2, p=0.042$; Fig. 3, A5, A6). Two-sided pairwise comparisons did not reach the adjusted critical a level of 0.017 (first and second night: $V=0, p=0.03$; first and third night: $V=1, p=0.0625$; second and third night: $V=7, p=0.56)$.

During the first night, translocated males remained close to the area where they were released (Fig. A5, A6). This pattern changed during the following two nights, and individuals travelled less often to the release position. Starting from the second night of translocation, five of six males made spacious forays, which strongly resembled the highly directed movements during dispersal forays (Fig. 1, A1, A2, A5 and A6). Maximum linear 
distance of these homing forays was $600 \mathrm{~m}$. Only one male returned to his habitual home range during the third night of translocation (Fig. A6), but he was only translocated over a distance of about one home range diameter $(200 \mathrm{~m})$. When the translocated male came close to the border of the home range of the neighboring individual during his second spacious foray, he changed direction and went directly back to his home range, traversing the home range of the apparently familiar individual (Fig. A6).

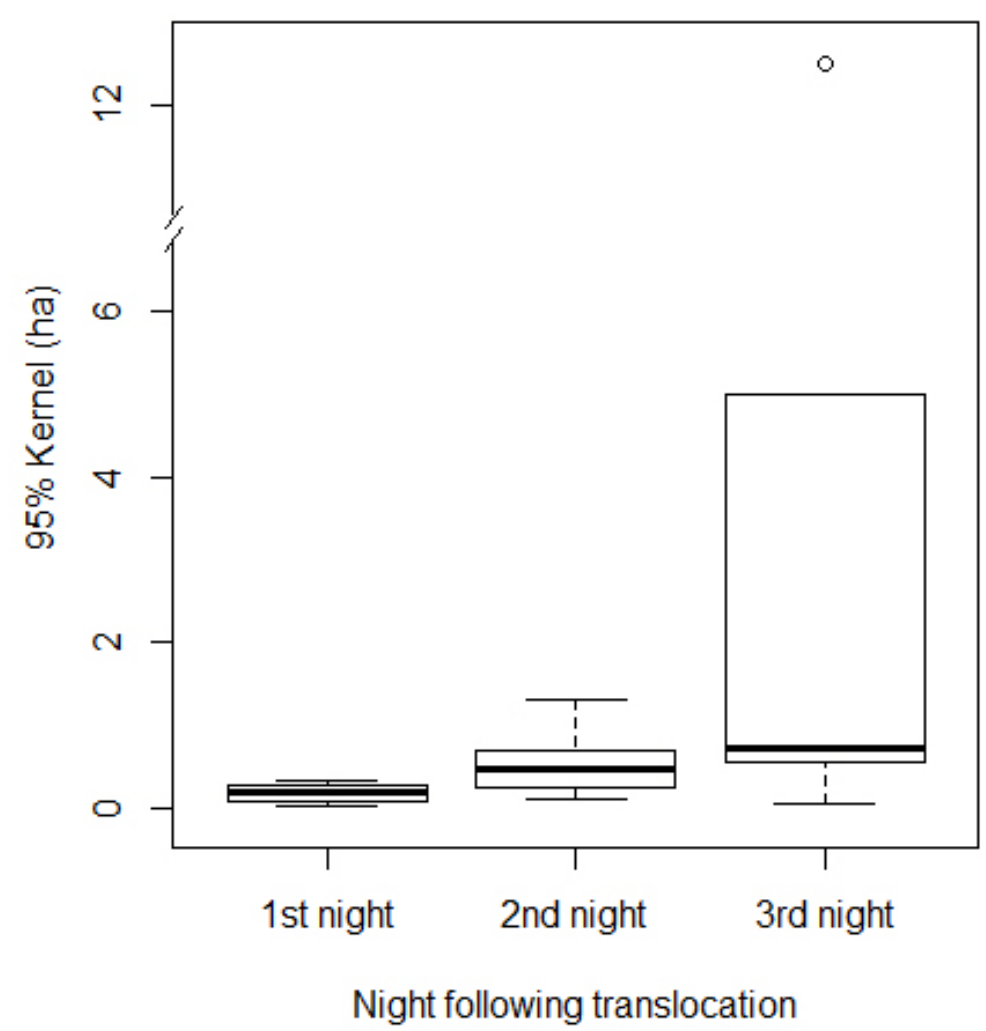

FIG. 3: SIZE OF PREVAILING RANGES OF 6 TRANSLOCATED MALES CALCULATED AS 95\%-KERNEL (HA)

Shown are median, interquartile range, max-min range and outliers. Size of home ranges was calculated based on temporally independent positions, collected for the respective nights. Prevailing ranges increased over time. A habitual home range of a subadult gray mouse lemur encompasses an area of about 1 ha.

\subsection{Average travel distances per hour}

Subadult males and females did not differ in average routine travel distances (Fig. 4, $W=322$, $p=0.24)$. During the first half of the night in the dry season, subadult males moved on average $203 \mathrm{~m} / \mathrm{h} \pm 64 \mathrm{~m}$ and subadult females about $183 \mathrm{~m} / \mathrm{h} \pm 77 \mathrm{~m}$. However, males did not move with the same travel velocity in all conditions (Fig. 4, Tab. 1, Kruskal-Wallis 
$\chi 2=19.64, d f=3, p<0.001)$. In fact, males doubled their travel distance per hour during dispersal (mean: $405 \mathrm{~m} / \mathrm{h} \pm 136 \mathrm{~m}$ ) in comparison to routine movements (Tab. 1, $p=0.001$ ).

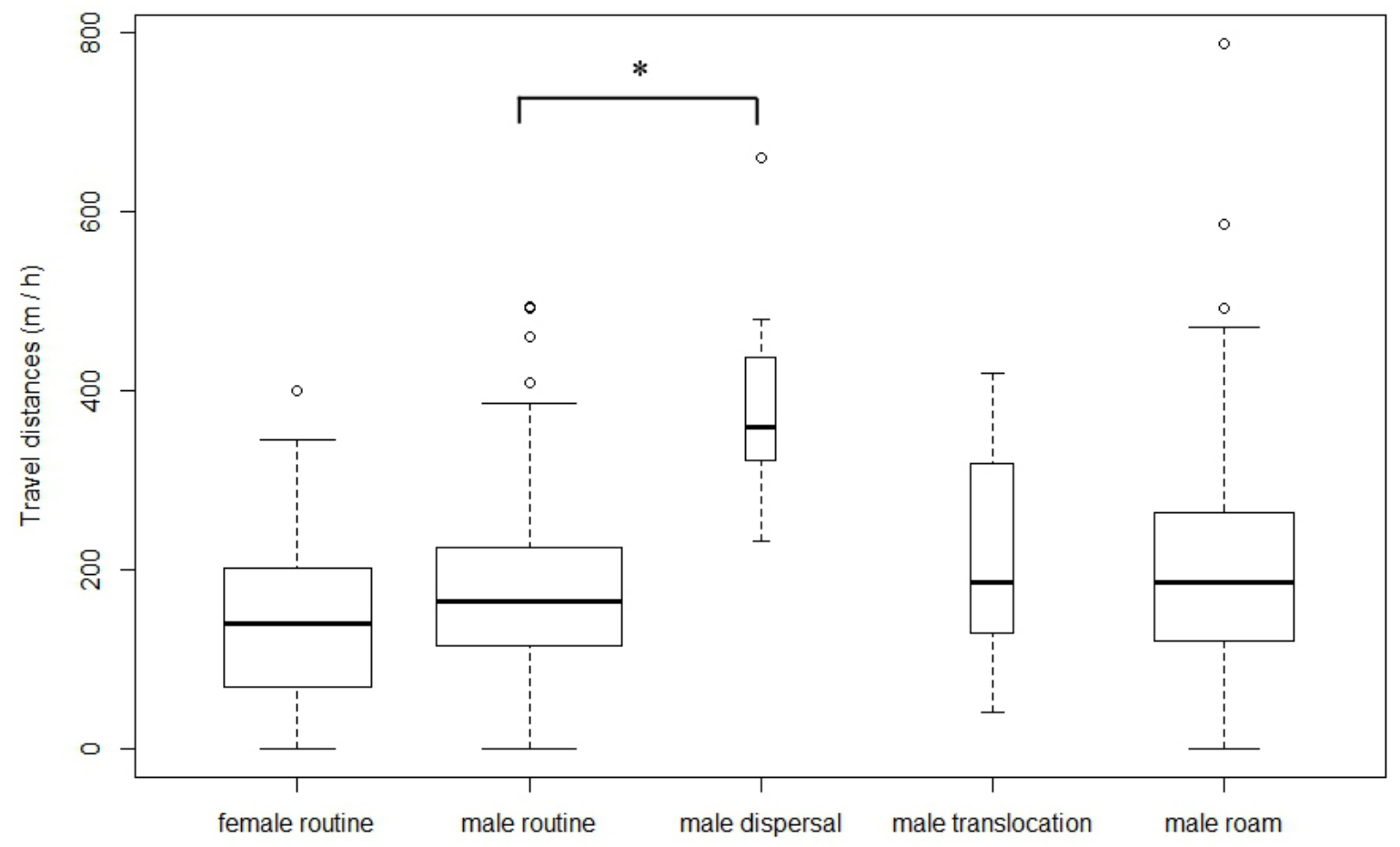

FIG. 4: STANDARDIZED TRAVEL DISTANCES PER HOUR FOR FEMALES DURING ROUTINE MOVEMENTS AND MALES DURING DIFFERENT SITUATIONS

For a comparison of travel distance, we used 204 observations for routine movements of subadult females $(\mathrm{N}=19), 329$ observation bouts of subadult males ( $N=33), 9$ observations of male dispersal pathways $(N=6), 188$ observations on male roaming pathways $(\mathrm{N}=19)$ and 18 data points of translocated males $(\mathrm{N}=6)$. Shown are median, interquartile range, max-min range and outliers, width of boxes indicates sample size. The bracket and star mark the significant difference between pairwise comparison of routine and dispersal movements. Remaining pairwise comparisons between male and female routine movements on the one hand and between male movements during different situations on the other hand revealed no differences.

TAB. 1: RESULTS OF DUNN'S POST HOC COMPARISON FOR AVERAGE TRAVEL DISTANCES/H FOR MALES

\begin{tabular}{lllll}
\hline & Dispersal & Routine & Roaming & Translocation \\
\hline Dispersal & - & & & \\
Routine & $6.67 \mathrm{E}-05^{*}$ & - & & \\
Roaming & 0.36 & 0.17 & - & \\
Translocation & 0.08 & 0.04 & 0.99 & -
\end{tabular}

Global $p$ value: $p=6.67 E-05 ; 99 \%$ confidence interval: lower boundary $=1.707713 E-0$, upper boundary $=1.739870 E-04$ 


\section{DISCUSSION}

Dispersal has been considered as a key behavioral mechanism in population biology and evolution, but it remains poorly documented in most species. Despite the unpredictability of this event and the practical difficulties of studying dispersal in a small, nocturnal, and arboreal mammal, we were able to collect data on behavioral aspects of natal dispersal for 12 individuals. Dispersal distances ranged from about one to seven home range diameters, and dispersal forays were behaviorally distinct from movements in other situations. Transfer was observed to last up to 14 days. Experimentally translocated individuals used the same linear movement strategy as during dispersal (homing forays). These data also imply that spatial knowledge of subadult gray mouse lemurs might be restricted to the familiar home range and that they lack a compass and or a map, because individuals that were translocated over short distances were not able to home back directly after release. Directions of dispersal and homing forays occurred in all cardinal directions. These aspects are discussed in detail below.

\subsection{What determines maximum dispersal distances?}

Knowing a species' range of possible dispersal distances is important for understanding the dynamics within and between populations. Short-distance dispersal is frequent and influences the composition of many attributes of a population, like abundance or relatedness, whereas long-distance dispersal seems to occur at lower frequencies and plays an important role in interpatch dispersal, recolonization, and invasion processes (Smith 1987; Koenig et al. 1996; Sutherland and Harestad 2000). During this study, individuals were able to relocate their home range within one night or in one movement step, overcoming distances of $180-960 \mathrm{~m}$. This distance range corresponds to the maximum dispersal distances detected by capture-recapture studies (Radespiel et al. 2003; Fredsted et al. 2005). Also, our results correspond to predicted dispersal distance based on a study investigating the influence of body mass and home range size on maximum possible dispersal distance (Bowman et al. 2002). However, whether the model can be used to predict maximum dispersal distance has still to be evaluated, because reported dispersal distances do not always match predicted ones. Reported dispersal distances of two other nocturnal primates, the Southern lesser bushbaby (Galago moholi, body mass, 100-250g, home range size, 7$11 \mathrm{ha}$ ) and the slow loris (Nycticebus coucang, average body mass, 685g, home range size, 
0.6-15ha), correspond to predicted values. Southern lesser bushbaby males dispersed over distances of $2,000 \mathrm{~m}$ in four to five consecutive nights, which is typical for many territorial species, since dispersers have to search for vacant territories and therefore move on if they cannot establish themselves in a certain area (Bearder 1987). Slow lorises dispersed up to 3,000m (Wiens and Zitzmann 2003). However, root voles (Microtus oeconomus; average body mass, 50g; reported max. dispersal distance, $3.2 \mathrm{~km}$ ), a terrestrial mammal of similar size to gray mouse lemurs, and pygmy rabbits (Brachylagus idahodensis; average body mass, $500 \mathrm{~g}$, reported max. dispersal distance, $6 \mathrm{~km}$ ) exceed maximum dispersal distance predicted for animals of their respective size by far (Steen 1994; Estes-Zumpf and Rachlow 2009). Thus, to determine what finally determines maximum dispersal distance, more observational data, capture-recapture or genetic studies with appropriate size of study area and sampling intensity are required.

\subsection{Movements in unfamiliar areas: dispersal and homing forays}

Dispersal movements differed considerably from routine or roaming movements. Dispersing gray mouse lemurs followed a rather straight line and covered larger distances per hour than during routine movements, while moving through unfamiliar habitats. Direction and distance of dispersal forays were consistent within individuals but differed between dispersers. Most strikingly, translocated gray mouse lemurs episodically fell back to the same mode of movements during translocation, which we interpreted as homing forays (cf. Lührs et al. 2009). Therefore, we think that these fast, directed movements are the standard exploration or dispersal strategy in gray mouse lemurs. Contrary to expectations (Van Dyck and Baguette 2005), that specialized dispersal movements do exclude dispersers from reacting to external cues, they did exploit resources during dispersal forays, even though they used a behaviorally distinct movement strategy to disperse. In combination with the fact that the presence of conspecifics plays a prominent role in the process of habitat choice (Stamps 2001), we think this working hypothesis should be revised to incorporate the possibility that decisions of dispersers are influenced by cues they encounter during transfer, even if they use special dispersal movements (Clobert et al. 2009). Comparable rapid straight line excursions have also been described for other species. Southern lesser bushbabies moved in a highly stereotypic, directed fashion during dispersal (Bearder 1987), and translocated males of this species also fell back to the same stereotypic movement mode (Bearder, 
personal communication). Other examples come from carabid beetles (Baars 1979), butterflies (Baker 1969), wolves (Mech 1987), red foxes (Storm et al. 1976), black bears (Rogers 1987), ground squirrels (Holekamp and Sherman 1989), and slow lorises (Wiens and Zitzmann 2003). Therefore, a variety of territorial and nonterritorial taxa with different types of social organization adopt this movement strategy during dispersal. But what could be the adaptive value of highly directed rapid dispersal movements?

One possible explanation for the observed dispersal movements of gray mouse lemurs is that linear movements represent an effective search strategy that offers reasonable information gain during transfer while balancing potential costs of dispersal movements. This assumption is based on theoretical models in which almost linear movements were the most adaptive and effective random searches, especially in situations with a high degree of landscape uniformity, high predation risk, and limited energy reserves (Zollner and Lima 1999; Conradt et al. 2003; Heinz and Strand 2006). Gray mouse lemurs conformed to some of these conditions, because predation risk is high for gray mouse lemurs (Rasoloarison et al. 1995) and they inhabit a range of different habitats (Rasoloarison et al. 2000). Moreover, concerning the gain of information, translocated individuals used the same movement strategy during homing forays to cover larger distances within unfamiliar areas. Therefore, observed dispersal movements might have evolved to balance dispersal costs and the need to gather information. However, the results of these models have to be transferred to gray mouse lemurs with caution because some of the underlying assumptions do not closely reflect the situation of a dispersing mouse lemur. These models often have to rely on unrealistic assumptions about costs and risks of dispersal, like an increased risk of starvation or predation, because they measure effectiveness of dispersal strategies as success probability of finding a suitable patch (Conradt et al. 2003). For gray mouse lemurs, the transfer phase can be an event of a few hours, so starvation is an unlikely cost. Instead, the benefits of getting familiar with the new site might be much more important for the fitness of gray mouse lemurs because of seasonality of food availability (Dammhahn and Kappeler 2008a; 2009; Lührs et al. 2009).

Alternatively, the question arises whether gray mouse lemurs need an elaborate systematic search strategy. A more parsimonious explanation might be that moving straight away from the natal range is a simple strategy to distance oneself from relatives in order to avoid inbreeding. Linear movements could allow individuals to cover larger distances in 
comparison to systematic search (Conradt et al. 2003), and they are feasible in a seemingly continuously suitable landscape. However, separation from relatives does not explain the variation in observed dispersal distance, and short-distance dispersers still lived close to their female relatives. Also, if linear movements were to present a lowly elaborated movement strategy which was not subjected to evolutionary trade-offs, it still remains difficult to explain how dispersers deal with the fact that the distribution of gray mouse lemurs is not homogeneous in continuous available habitat (Fredsted et al. 2004). It therefore seems unlikely that there are no further benefits connected to this movement strategy.

Our data on translocation movements point towards another possible constraint that might favor the observed dispersal strategy. For gray mouse lemurs, spatial knowledge of subadults might be restricted to their own home range because even over very short translocation distances, they were unable to home directly back to their habitual home range. Prevailing areas during translocation increased for all individuals from the first to the second and/or third night with decreasing frequency of moving to the area of release, indicating that spatial knowledge is accumulated by moving through a certain area (Fig. 3). With increasing experience with a site, the navigational abilities and effectiveness of an individual probably also improve (Joly and Zimmermann 2011). A study on the spatial memory abilities of gray mouse lemurs (Lührs et al. 2009) suggested that they have a mental representation that is more detailed than a network of routes and landmarks, referred to as route-based network (Byrne 1979). This way of orientation relies strongly on experience, because individuals need to form a spatial representation of traversable paths. For dispersal movements, this form of orientation could restrict the degree of tortuosity of dispersal movements if tortuosity compromises the option of returning to the old home range. Because gray mouse lemurs mark their home range with saliva, anal secretions, and urine, dispersers may use these odors as a source of information guiding their movements (Schilling 1979). Conspecifics' or individuals' own scent marks could also serve as landmarks that facilitate route reversal.

\subsection{Why commute?}

Commuting between old and new home range during the transfer phase seemed to play an important role for some gray mouse lemurs during dispersal. Some individuals extended 
their transfer phase up to 14 days. Extended periods of commuting are also known for other species, like European badgers (Roper et al. 2003) or dwarf mongooses (Rood 1987). We think the advantage of commuting between two areas is the possibility to explore the physical and social settings of the new site while still relying on resources at the old site. The individuals of our study population mainly feed on gum during the time of dispersal (Dammhahn and Kappeler 2008a). Since they have to learn where to find gum trees in the new home range, it seems advantageous not to abandon the old home range before having gained enough knowledge about the new site, especially when competing with residents for these crucial resources (Génin 2003; Lawson Handley and Perrin 2007; Del Mar Delgado et al. 2009; Lührs et al. 2009). Also, individuals responded to resources that they encountered on their forays (Fig. A2). Therefore, commuting is one option for dispersers to reduce costs or avert fatal consequences of dispersal.

\subsection{Dispersal direction}

Directions of dispersal and homing forays in gray mouse lemurs occurred in all cardinal directions. However, there seemed to be a higher preference to disperse towards the eastern and northeastern part of our study site. This tendency needs to be corroborated through additional data, analyzing the relationship between gradients of habitats and population densities for gray mouse lemurs and dispersal directions. Constraints on dispersal directions on a population level have also been described for pygmy rabbits, where landscape features limit directions (Estes-Zumpf and Rachlow 2009).

Concerning the direction of individual dispersal trajectories, at this point, it remains unknown how gray mouse lemur males decide in which direction to go and where to stop during dispersal or homing forays. Though unlikely, we cannot fully exclude the possibility that subadult males made undetected dispersal forays or explored the surroundings of their natal home range, as has been described for North American red squirrels (Tarniasciurus hudsonicils: (Larsen and Boutin 1994). Direction of dispersal can also be chosen spontaneously without prior exploration of alternative options (Smith 1974). If dispersers chose direction randomly, it remains unclear why individuals become inflexible once they have chosen a direction, because unsuccessful dispersers never changed the direction of their dispersal forays. One option for individuals to modulate dispersal success could then be to adjust dispersal distances whenever they encounter a gradient in habitat suitability. Our 
observation of a disperser that moved first beyond the point where it established its home range and subsequently reduced linear distance between old and new home range might indicate such a modulation.

\section{CONCLUSION}

Although there has been much progress in identifying general dispersal trends via genetic studies, species or individual dispersal abilities and processes have virtually remained a black box. We need further empirical studies of a variety of species that differ with respect to their social organization and spatial tolerance to better understand how dispersal strategies and distances evolved. The present study is one of only a few describing detailed dispersal movements under natural conditions. The prominence of highly directed movements in a variety of taxa suggests general advantages of this dispersal strategy. One advantage of this movement strategy seems to be the opportunity to commute between old and new home ranges relatively easily, which in turn allows dispersers to mitigate costs of unfamiliarity. Thus, unsuccessful dispersal does not necessarily have fatal consequences, which is usually assumed in modeling approaches.

\section{ACKNOWLEDGEMENTS}

We acknowledge the authorization of this study by the Commission Tripartite and the CAFF of the Direction des Eaux et Forêts and the CNFEREF. The Courant Research Centre "Evolution of Social Behaviour" (CRCESB) and the Christian-Vogel-Fonds (GfP) provided financial support. We thank Dr. Rodin Rasoloarison, Léonard Razafimanantsoa, Tiana Andrianjanahary, Bruno Tsiveramana, Remy de Ampataka, and the Equipe Kirindy for support and assistance in the field, and Dr. Melanie Dammhahn for helpful comments on earlier versions of the manuscript. A previous version of the manuscript benefited greatly from comments by three anonymous referees.

\section{ETHICAL STANDARDS}

All research reported here is in compliance with animal care regulations and applicable national laws of Germany and Madagascar. The appropriate animal use and care committees of Germany (Bundesministerium für Naturschutz, BfN) and Madagascar (Ministère de I'Environnement et des Eaux et Forêts, MINEEF) approved all research protocols. 


\section{APPENDIX A: SUPPLEMENTARY FIGURES}

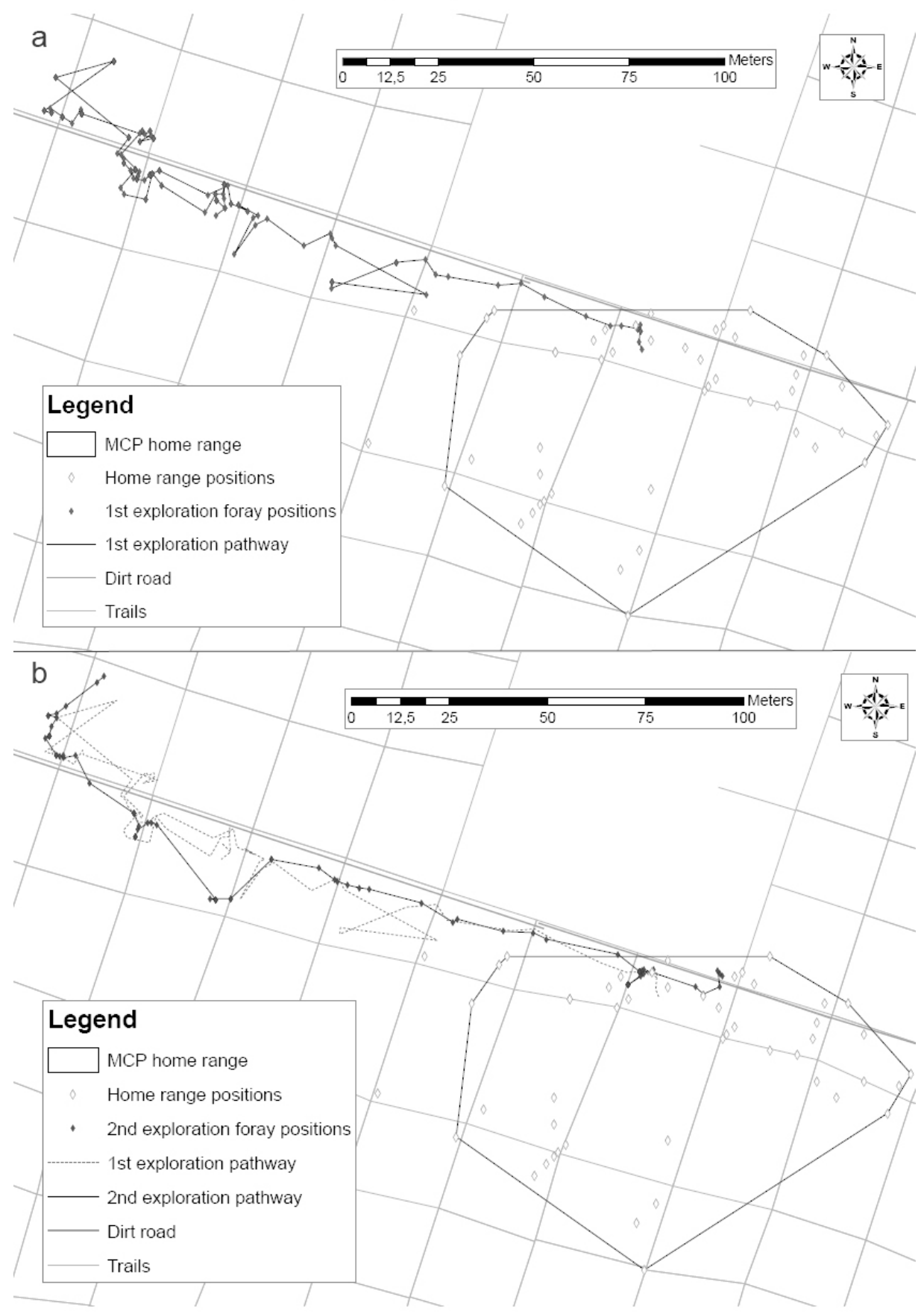

FIG. A1: EXPLORATION FORAYS OF UNSUCCESSFUL DISPERSER C404

(a) First recorded exploration foray

The figure shows a section of the gridsystem. The natal home range of the individual is indicated by a $95 \%$ $\mathrm{MCP}$, which was calculated based on 50 temporally independent locations (white diamonds) chosen randomly from 196 data points. Gray diamonds show recorded positions during the exploration foray, which we attempted to collect every minute (no. positions: 127 , observation time: $148 \mathrm{~min}$ ). The solid line connecting them shows the pathway.

(b) Second recorded exploration foray

The second exploration foray occurred one week after the first exploration foray. Gray diamonds show again positions during the exploration foray and the solid line shows the pathway of the second exploration foray (no. positions: 76 , collected within $84 \mathrm{~min}$ ). The dashed, gray line indicates the route for the first exploration foray. 


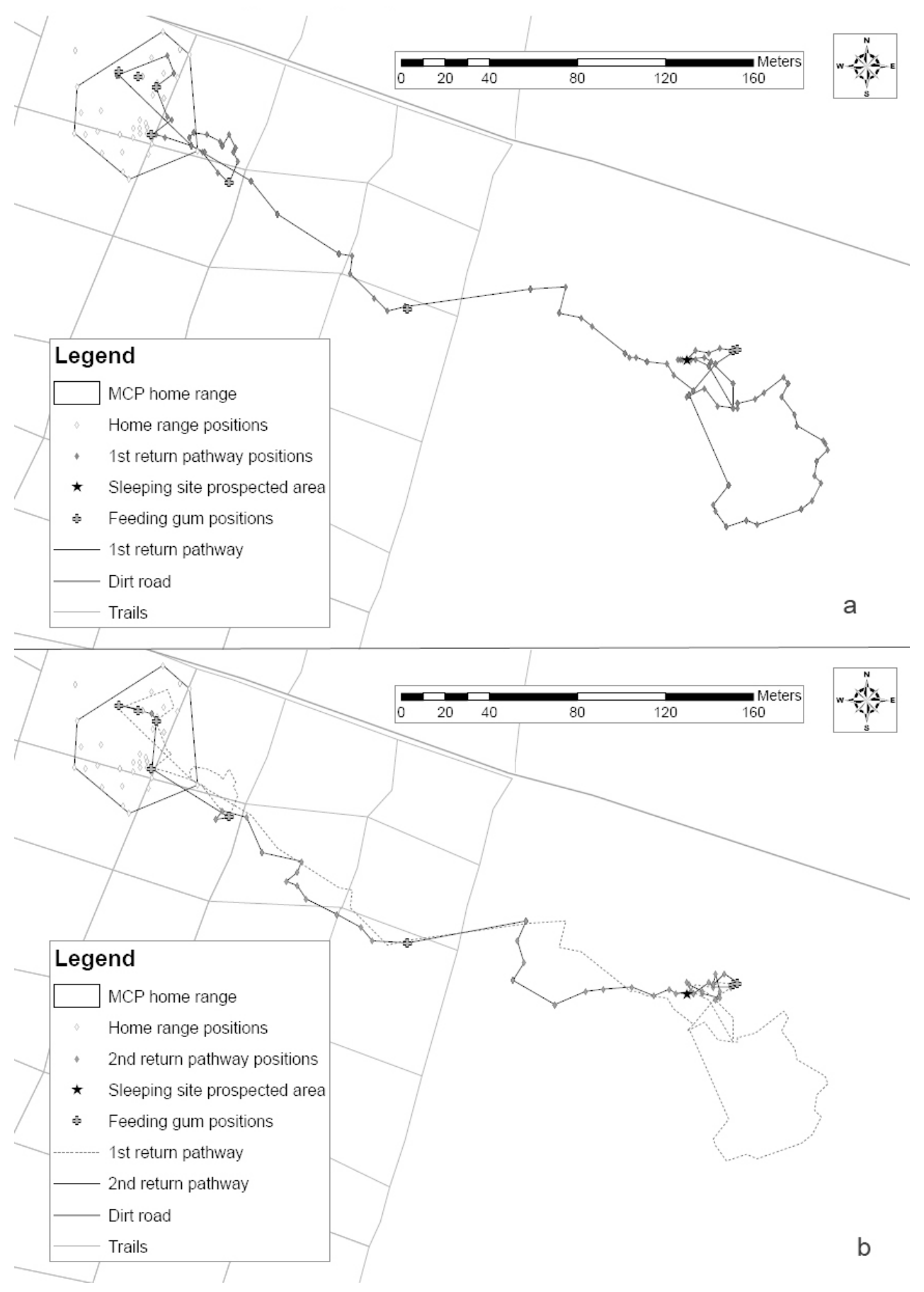

FIG.A2: RETURN PATHWAYS OF UNSUCCESSFUL DISPERSER 4B8E

(a) The figure shows a section of the gridsystem. The natal home range of the individual is indicated by a $95 \%$ MCP, which was calculated based on 50 temporally independent locations (white diamonds) chosen randomly from 75 data points. The solid line connecting the gray diamonds shows the return pathway (no. positions: 185). The individual had slept at the prospected site (black star) and was followed from the moment when it started its activity. After initial exploration of the new area, the animal eventually returned to its natal area. Gray crosses represent positions where the individual stopped to feed on gum. The individual was found to have returned back to the prospected site within the same night.

(b) The second return pathway was recorded the following night. After returning to the prospected area the night before it used the same sleeping site (black star). Again, we followed the individual from the moment activity started (no. positions: 81). This was the last incident during which this individual was observed to move outside its natal area. 


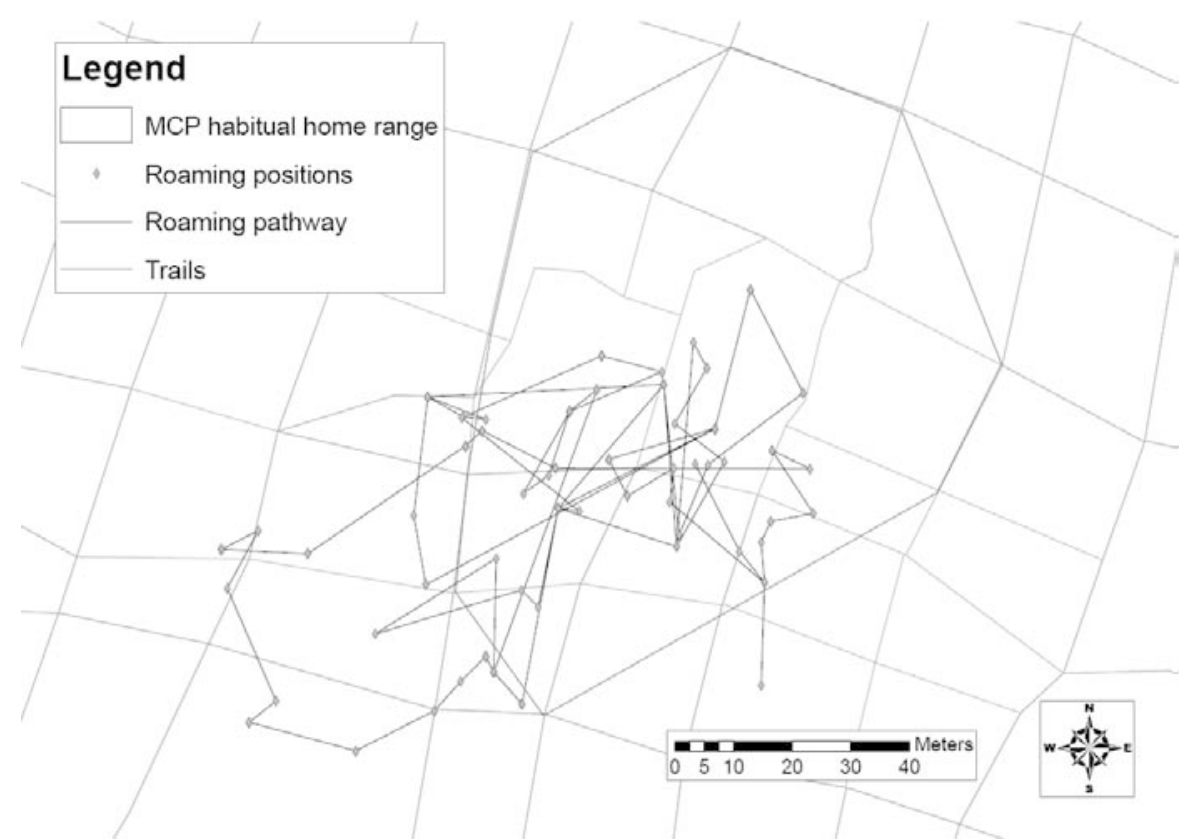

FIG. A3: ROAMING PATHWAY OF A ONE-YEAR OLD MALE DURING MATING SEASON

The figure shows a section of the gridsystem. Illustrated is a pathway of one year old male during mating season in the year 2000. The male appeared at our study site in April 2000 and was present until October 2003. In contrast to dispersal movements, roaming movements lack the high degree of linearity. The habitual home range of the individual is indicated by a $95 \%-\mathrm{MCP}$, which was calculated based on trapping data. For this, we used positions of 42 trapping events collected outside the mating season between 2000 and 2003

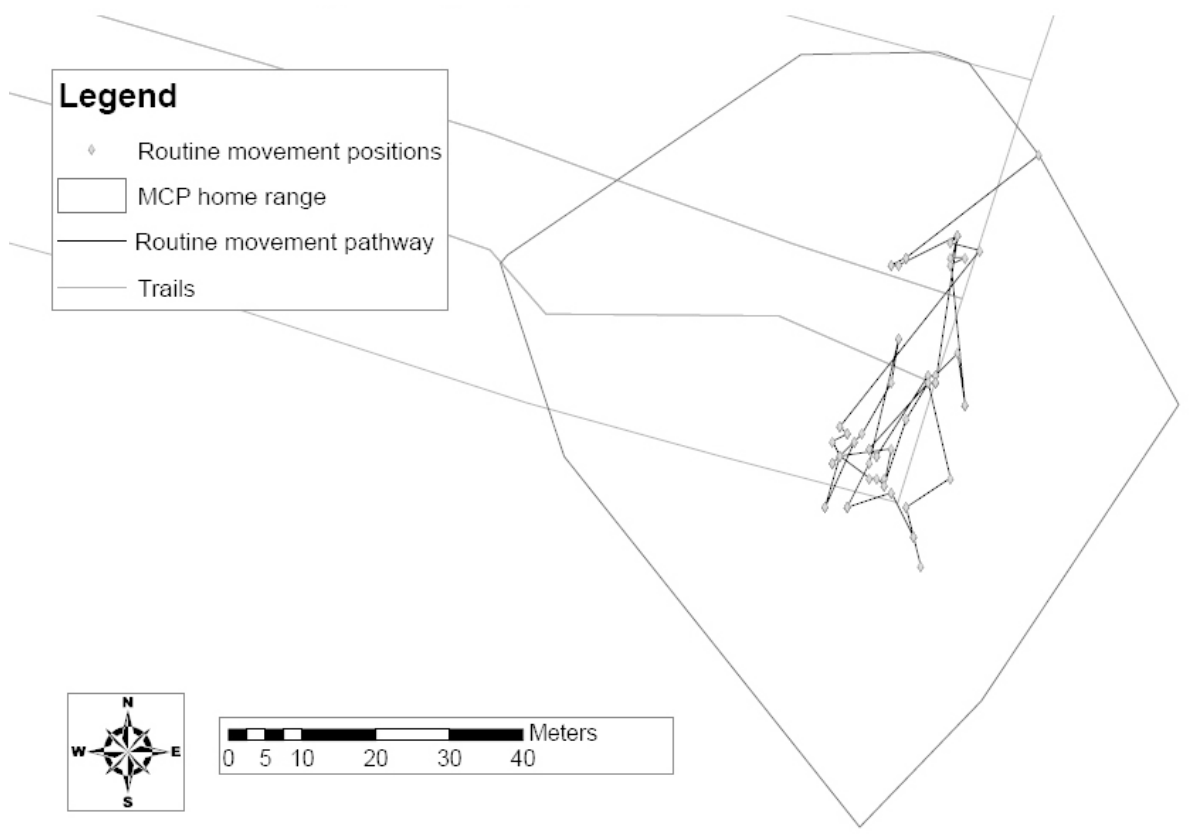

FIG. A4: MOVEMENT PATHWAY OF SUBADULT MALE WITHIN ITS HABITUAL HOME RANGE

The figure shows a section of the gridsystem. Illustrated are routine movements (collected during a 40min observation) for a subadult male during the dry season in the year 2010. Data were collected for the post dispersal phase of the individual. The habitual home range of the individual is indicated by a $95 \%-\mathrm{MCP}$, calculated from 50 independent tracking locations. 


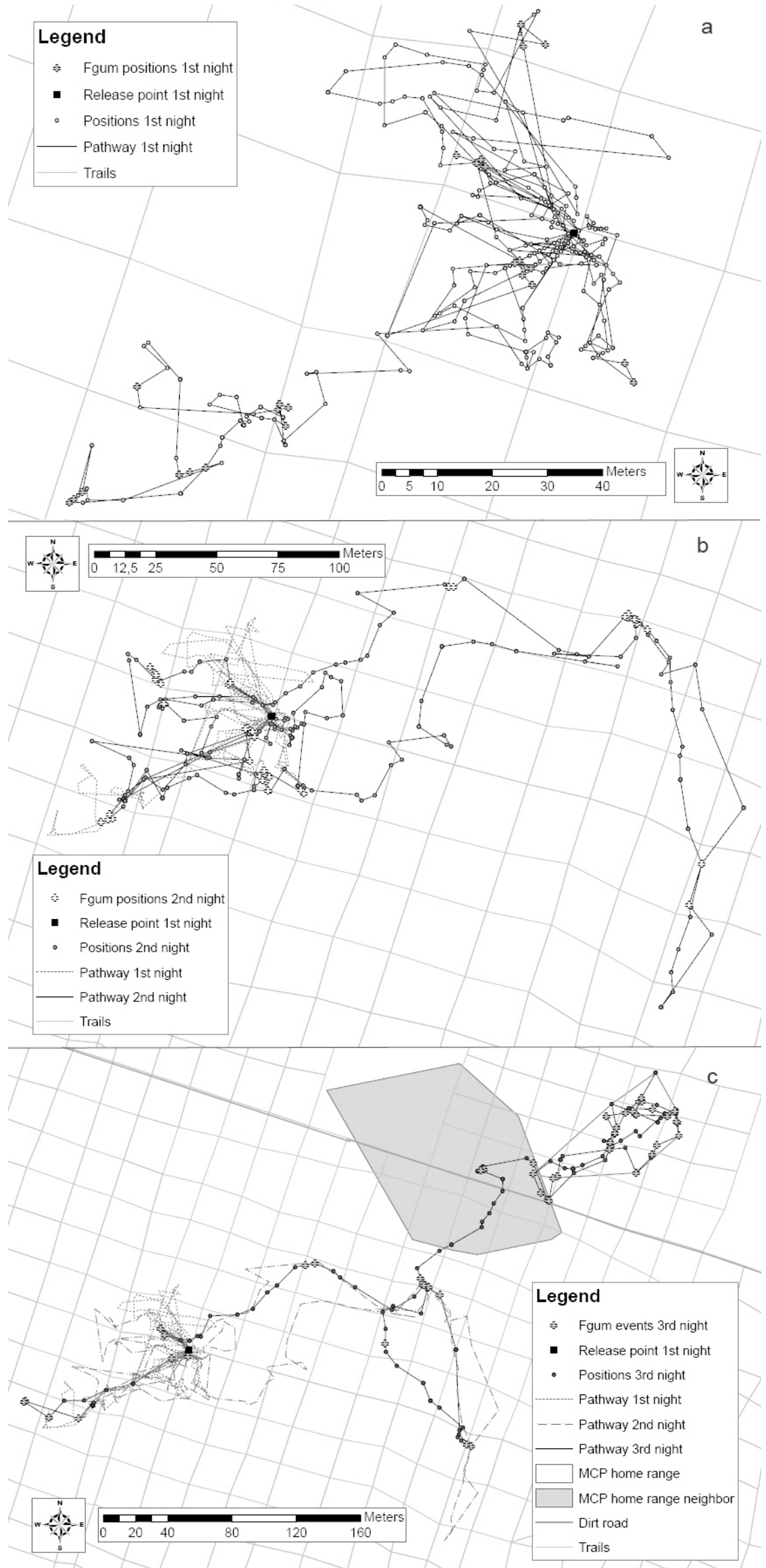

FIG. A5: MOVEMENT PATHWAY DURING THREE NIGHTS OF TRANSLOCATION OF MALE E140 
(FIG. A5: CONTINUED)

(a) The figure shows a section of the gridsystem. The male was translocated over a distance of $200 \mathrm{~m}$. It gradually increased the explored area using the area around the position where we released it (black square) as a base station

(b) The individual headed for the release position (black square) less often. Instead it made a spacious exploration foray away from the release site. Notably, it returned almost on the same pathway to the release area, instead of navigating over a shorter distance.

(c) The individual made a foray to the same area as in the night before, using more or less the same pathway. On the way back, the male changed direction and returned to its habitual home range. The change of direction occurred close to the border of the home range of a neighbouring male (home ranges indicated as 95\%-MCP, calculated based on all available spatial data). 


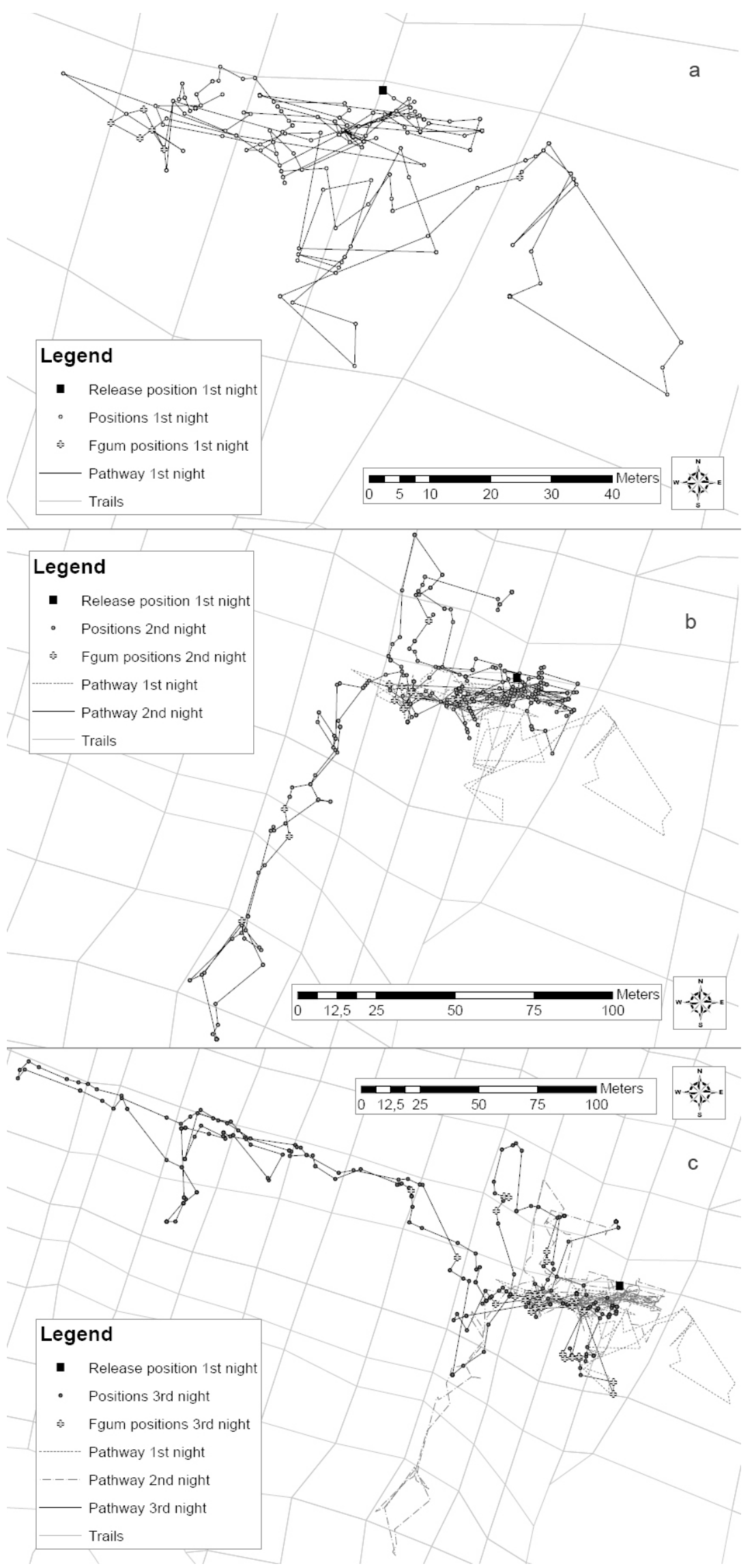

FIG. A6: MOVEMENT PATHWAY DURING THREE NIGHTS OF TRANSLOCATION OF MALE 651E 
(FIG. A6: CONTINUED)

(a) The figure shows a section of the gridsystem. The male was relocated over a distance of $300 \mathrm{~m}$. The used area was increased gradually, but the individual remained close to the area where we released it.

(b) The individual started to make spacious homing forays away from the release site. Movements strongly resembled dispersal forays.

(c) In contrast to dispersal movements, the direction of homing forays was not fixed 
Chapter 2: Proximate Mechanisms of DisPersal

\author{
Ready to go? \\ On the relationship between \\ natal dispersal and body mass \\ in gray mouse lemurs (Microcebus murinus)
}

with Manfred Eberle and Peter M. Kappeler 


\section{ABSTRACT}

Condition-dependent dispersal allows individuals to match this important life history event to internal and external cues. A basic component of condition-dependent dispersal is physical condition because of dispersal-related energetic costs. Good physical condition is usually regarded as a safe guard in this context. We investigated whether and how body mass influences dispersal propensity and dispersal distances of male gray mouse lemurs (Microcebus murinus), small, nocturnal, solitary primates from Madagascar. Based on pedigree and trapping data collected between 1994 and 2010, we established that gray mouse lemurs require a minimum body mass of about $35 \mathrm{~g}$ before dispersing. However, we found no evidence for a link between dispersal distance and body mass or body condition. High flexibility of dispersal distance with respect to body condition might be a consequence of patchy distribution of local gray mouse lemur population nuclei. Thus, while body mass has a deterministic influence on dispersal propensity, complex interactions of other proximate factors subsequently determine the exact timing and the distance travelled during natal dispersal in this species.

Keywords: condition-dependent dispersal, dispersal timing, natal dispersal, Microcebus murinus

\section{INTRODUCTION}

Dispersal is a key element of an individual's life history and holds a central role for the dynamics and evolution of populations, because it affects the distribution of organisms in space and time (Ronce 2007). Departure from the birth place or group (natal dispersal) is often timed around sexual maturation and sex-biased in many vertebrates (Greenwood 1980). The proximate mechanisms underlying these tendencies remain poorly understood (Smale et al. 1997; Dufty and Belthoff 2001; Dufty et al. 2002). When natal dispersal rates are strongly sex-biased, genetic and physiological factors are most likely involved in controlling the dispersal of individuals (Lidicker and Stenseth 1992). Although studies invoking genetic elements involved in sex-specific dispersal propensities remain scarce, this link has been demonstrated in a few insects (Roff and Fairbairn 2001; Haag et al. 2005) and vertebrates (Trefilov et al. 2000; Krackow 2003; Massot et al. 2003). One important insight 
from these studies is that the proximate effects of genes and hormones on dispersal propensity typically allow for a certain degree of flexibility, especially concerning the exact timing of individual dispersal.

Flexibility in behavioral response in this and other contexts has been regarded as a strategy to match internal state and environmental condition (Dufty and Belthoff 2001; Ims and Hjermann 2001; Massot et al. 2002; Le Galliard et al. 2012). Internal or phenotypic conditions comprise genetic constitution (Kaplan et al. 1995; Haag et al. 2005; Selonen and Hanski 2010), physical condition (Nunes et al. 1998; Edelman 2011), hormone levels (Dufty and Belthoff 2001; Dufty et al. 2002; De Sousa et al. 2009; Quirici et al. 2011) and personality (Hoset et al. 2011; Cote et al. 2012), whereas environmental conditions encompass habitat quality (Lurz et al. 1997), population density (Delibes et al. 2001; Gundersen et al. 2001; Matthysen 2005), maternal effects (Massot et al. 2002) and social environment and stability (Alberts and Altmann 1995a; Long et al. 2008; Jack et al. 2011).

The ubiquity of condition dependent dispersal strategies indicates its advantages over inflexible strategies, which stems from a reduction of costs and increased benefits (Alberts and Altmann 1995a; Bowler and Benton 2005; Bonte and De La Peña 2009; Armitage et al. 2011; Edelman 2011; Bonte et al. 2012; Le Galliard et al. 2012). These costs have been described in a number of studies and can include decreased survival because of increased predation risk (Van Vuren and Armitage 1994; Yoder et al. 2004), increased energetic costs and decreased foraging efficiency (Boinski et al. 2005; Bonte et al. 2012), the separation from kin as potential coalition partners (Isbell and Van Vuren 1996), aggression by conspecifics (Smith 1987; Smale et al. 1997), missed opportunities of reproduction (Alberts and Altmann 1995a) and poor habitat choice (Delibes et al. 2001). Consequently, the dispersal process of an individual is often described as a plastic process resulting from a complex interaction between internal and external factors (Murren et al. 2001; Dufty et al. 2002; Massot et al. 2002; Clobert et al. 2009).

Although the exact physiological mechanisms underlying natal dispersal behavior remain poorly understood, a central role in the timing of dispersal has been attributed to body condition (BC), since one of the most important sources of dispersal costs stems from reduced feeding efficiency caused by unfamiliarity with the new environment and increased energy expenditures through higher travel activity (Dufty and Belthoff 2001; Bonte et al. 2012). In some species, the higher travel activity is part of a dispersal-related behavioral 
syndrome, which can also be associated with short-termed changes in personalities (Holekamp 1986; Belthoff and Dufty 1998; Hoset et al. 2011). In these cases, BC-dependent dispersal serves most likely as a safeguard for the transient and settlement stage of dispersal, but possibly also as a bet-hedging strategy for variable environments or poor habitat choice (Delibes et al. 2001; Bonte and De La Peña 2009; Bonte et al. 2012). For example, in female chimpanzees, BC impacts emigration timing (Stumpf et al. 2009). In naked mole-rats, dispersal is preceded by a growth spurt and potential dispersers have higher body mass and changed hormone profiles (O'Riain and Braude 2001). Experimental food supplementation of banner-tailed kangaroo rats increased growth rates and let to earlier dispersal of males (Edelman 2011). In ground squirrels, body fat content interacts with endogenous timing mechanism, so that males with low body fat content postpone dispersal (Holekamp and Sherman 1989; Nunes and Holekamp 1996; Nunes et al. 1998). In yellow-bellied marmots yearling male delay dispersal when they are underweight (Downhower and Armitage 1981). And in red squirrels, settlement success is linked to body mass (Wauters et al. 1993). Therefore, physical condition represents a measure for developmental state, which can determine the activation of the dispersal process (Alberts and Altmann 1995b).

However, BC does not always show a positive association with dispersal propensity (Bowler and Benton 2005). For example, while increasing BC increased dispersal propensity of juvenile common lizards (Meylan et al. 2002) and greater flamingos (Barbraud et al. 2003), juvenile dwarf spiders (Mestre and Bonte 2012) and adult female red squirrels (Wauters et al. 1995) dispersed more readily in response to food deprivation or moved to territories of better quality. Similarly, when high benefits of philopatry are combined with high potential for competition, individuals with low BC disperse (or get displaced) more frequently or over larger distances (Tucker et al. 1998; Dufty and Belthoff 2001; Bonte and De La Peña 2009).

Nevertheless, species with positive BC dependent dispersal should have a threshold of minimum required body size or mass that reflects the level of maturation and whether an individual is able to disperse or not (Alberts and Altmann 1995b; Smale et al. 1997; Belthoff and Dufty 1998; Nunes et al. 1998; Edelman 2011). Additionally, if dispersal is voluntary and not linked to displacement because of lower competitive abilities, it seems plausible that a similar link leads to BC dependent dispersal distances since increased dispersal distances could be associated with increased risks and energetic costs (Murray 1967; Rousset and 
Gandon 2002). However, this positive association depends also on the requirements of an individual and is probably not true for species that are dependent on limited key resources, like mounds in kangaroo rats (Edelman 2011) or middens in red squirrels (Larsen and Boutin 1994). Both are used for food storage in these species and individuals usually disperse to the nearest available site.

In the present study, we investigated the role of body mass in the timing of natal dispersal and its effects on dispersal distances of a small, solitary Malagasy primate, the gray mouse lemur (Microcebus murinus). Gray mouse lemurs present a good model to examine interaction between body condition and dispersal propensity for several reasons. First, it is well established that several life history traits of mouse lemurs are linked to body mass: The ability to use hibernation and torpor for females and males requires a minimum body mass (Schmid 1999), and the rearing success of females (Eberle and Kappeler 2004a) as well as male mating success (Eberle and Kappeler 2004b) increase with body mass. Second, the life cycles of gray mouse lemurs are closely tuned to the seasonality of their habitat. In the dry deciduous forests of Madagascar, all events of reproduction - mating, birthing and rearing of offspring - occur (usually) during the wet season, lasting from November to March, when food availability and quality is highest (Eberle and Kappeler 2004b; 2006; Dammhahn and Kappeler 2008a). Adult body masses vary between 50 and $120 \mathrm{~g}$ and fluctuate depending on season (Schmid and Kappeler 1998; Dammhahn and Kappeler 2008a). Body mass of neonates is about 6g, but this value can be tripled within 10 days in captivity (Perret 1990). Independent foraging of subadults in wild populations starts at an age of about 2 months, and male-biased natal dispersal starts with the transition towards the dry season (Radespiel et al. 2003; Fredsted et al. 2005; Eberle and Kappeler 2006). Female dispersal occurs only at very low frequencies and also over shorter distances (Radespiel et al. 2003). Studies on captive gray mouse lemurs revealed that bone growth is strongest during the first six months of life, and lines of arrested growth in long bones coincide with seasonal transitions and possibly weaning (Castanet et al. 2004). Therefore, the time of male dispersal coincides with a period of declining food availability and incomplete growth. Since male gray mouse lemurs provide no parental care, their timing of natal dispersal should be closely related to body size and BC to increase their probability of successful dispersal (Edelman 2011).

Against this background, we tested whether flexibility in timing of dispersal is related to variation in body mass and condition in male gray mouse lemurs based on capture-mark- 
recapture data collected between 1994 and 2010. We used pedigree and parentage data generated for the same time period to determine the dispersal state and natal origin of males. Moreover, we compared body mass of subadult males and females to test for sexdifferences in developmental patterns. Furthermore, we related body mass and condition to observed dispersal distances. Together, these analyses illuminate proximate trade-offs and determinants of dispersal in a primate species that deviates in many life history and social traits from better-studied anthropoids and therefore informs comparative studies of both mammals in general and primates in particular.

\section{METHODS}

\subsection{Study site and capture methods}

The study population is located within a 12,500 ha forestry concession of the Centre National de Formation, d'Etude et de Recherche en Environnement et Foresterie (CNFFREF) in Kirindy Forest (Kappeler and Fichtel 2012a). Since 1994, DNA samples and population parameters have been collected during regular captures in an area of about 9ha within a 60ha grid system, locally known as CS7. Additional captures in surrounding areas were conducted once or twice a year and covered an additional area of 18ha. Trapping procedures and animal handling have been described in Eberle and Kappeler (2002).

\subsection{Parentage analyses for determination of natal origin}

12 different polymorphic microsatellite systems with an average number of 22 alleles (range: 13-39) were used for parentage analyses (Mm06, MmF3, Pvc 9.2, Pvc a.1: Wimmer et al. 2002; 33104, Mm22, Mm39, Mm40, Mm42, Mm43b, Mm51, Mm60: Hapke et al. 2003). Microsatellite loci were checked for the presence of null alleles, stutter errors or short allele dominance with the program MICRO-CHECKER v. 2.2.3 (Van Oosterhout et al. 2004). Parentage analysis based on combined mismatch and likelihood analysis was performed with CERVUS 3 (Kalinowski et al. 2007) and COLONY v. 2.0.1.9 (Jones and Wang 2010). To sort candidate parents, a maximum of two homozygous mismatches or one heterozygous mismatch was accepted. The likelihood analysis for non-excluded candidates in CERVUS 3 was based on detailed parentage simulations (100,000 runs, 94 candidate parents, assumptions: sampling rate $=0.95$; average loci typing rate $=0.85$; error rate $=0.05$; one close relative of the true parent among the other candidate parents) to estimate the resolving 
power of all loci and to estimate critical values to evaluate the parentage analysis statistically.

\subsection{Dispersal status and distance}

One prerequisite for determining dispersal status (yes/no) of subadult individuals was that the mother could be identified. Based on parentage analyses for about 1,100 individuals, we could identify the mothers of 159 males and 184 females. For these individuals, we compared the linear distance between average trapping location of them and of their mothers. For individuals to qualify as dispersers, we set a minimum required distance between these trapping locations of $150 \mathrm{~m}$, which corresponds to an average home range diameter (Eberle and Kappeler 2002). Since dispersal movements of gray mouse lemurs are almost linear (Chapter 1), the linear distance between the coordinates of mean trapping locations of mother and offspring was used as a measure of dispersal distance.

For individuals with identified mothers that did not qualify as dispersers because distances between average trapping locations was smaller than $150 \mathrm{~m}$, we determined how long they were present in the core study area. If they continued to be present until the second year of their life without shifting their home range, we classified them as philopatric. If they disappeared before, their dispersal status remained undetermined because their absence could be the result of dispersal or death.

\subsection{Morphometric data}

We included into our analyses capture data collected from subadult individuals (with identified mother) between March and November from the years 1994-2010. Routine procedures during captures included weighing individuals at least once per month, but morphometric measurements were taken on a less regular basis. However, measurements of body length, head length and head width were highly positively correlated with body mass for subadult gray mouse lemurs ( $r=0.797,0.793$ and 0.824 respectively, Fig. 1). Therefore, we used body mass as a proxy for developmental state and relative BC, but controlled our results by repeating calculations with smaller data sets for BC. BC was calculated as body mass [g]/head width [mm] (Dammhahn and Kappeler 2009). Data were separated into trapping events prior to dispersal (pre-dispersal state) and after dispersal (post-dispersal state), respectively. 


\subsection{Statistical analyses}

First, we assessed the presence/absence of general sex differences in body mass. Because of non-normality in the distribution, we used the unpaired Wilcoxon rank sum test. To account for possible seasonal changes in body mass, we repeated the test for three different time periods: March-May (transition to dry season, TDS), June-August (dry season, DS) and September-November (transition to rainy season, TRS), which reflect broad differences in food availability and temperature, with lowest food availability and temperatures during DS. TRS includes the mating season. For this analysis, we averaged individual values per season.

We evaluated whether body mass and BC predicted an individual's dispersal state (pre-dispersal or post-dispersal) using GLMM (family: binomial) calculated in R v. 2.15.0 (CThe R Foundation for Statistical Computing) with the package Ime4 (Bates et al. 2011). We included as explanatory variable body mass or BC measured per month interacting with 'season' to control for seasonal effects. Data on pre-dispersal body masses and condition included also data from individuals with undetermined dispersal state or philopatric individuals. When we included all repeated measures of individuals, low number of recorded transitions between the two dispersal states caused a high degree of underdispersion within the data set. Therefore, we restricted our analysis to the latest measures available for the pre-dispersal state and the first measures available for the post-dispersal phase. Since sample size of females was low, they were omitted from these analyses.

Assessing the relationship between $\mathrm{BC}$ and dispersal distances based on trapping data proved to be rather difficult. The resolution of trapping data was in most cases insufficient or too coarse to be able to measure changes in BC caused by dispersal. For 21 of 65 individuals, we could determine the exact month of dispersal. For the remaining dispersers, we could only establish an estimated maximum month of immigration. Therefore, we used a different approach. Instead of investigating individual changes, we evaluated the relationship between body mass and dispersal distance using linear models for data of the pre- and post-dispersal phase separately. Assuming that costs of dispersal increase with increasing dispersal distance, pre-dispersal body mass should show a positive association with dispersal distance, whereas post-dispersal body mass should show a less positive or even a negative association (Rousset and Gandon 2002). We repeated this analysis using BC as predictor for dispersal distance. Analyses were performed using the 
'stats' package (C) R Core Team and contributors worldwide) in R v. 2.15.0 (CThe $R$ Foundation for Statistical Computing).

\section{RESULTS}

\subsection{Relationship between dispersal state and body mass}

Body masses of subadult males and females measured during the three seasons were compared for 260, 153 and 159 individuals with a female-male ratio of 1.13, 1.04 and 1.6, respectively. Within seasons, we could not detect any marked sex differences in body mass (Fig.1, TDS: $n_{\text {females }}=138, n_{\text {males }}=122, W=9181, p=0.20 ;$ DS: $n_{\text {females }}=78, n_{\text {males }}=75, W=3355$, $p=0.11 ;$ TRS: $n_{\text {females }}=98, n_{\text {males }}=61, W=2812, p=0.42$ ).

The probability of males to be in either dispersal state was evaluated based on 172 data points for body mass ( $n_{\text {pre-dispersal }}=120, n_{\text {post-dispersal }}=52$ ) and for 103 data points for BC $\left(n_{\text {pre-dispersal }}=66, n_{\text {post-dispersal }}=37\right)$. For males, a high body mass increased the probability that a male was in the post-dispersal state significantly (estimate $=0.12, S E=0.04, z=2.89, p=0.003$, Fig. 2, 3, Tab. 1). The lowest post-dispersal body mass per season was $36 \mathrm{~g}$ for TDS, $39 \mathrm{~g}$ for DS and $46 \mathrm{~g}$ for TRS, indicating a threshold of minimum body mass for dispersal around $35 \mathrm{~g}$. No seasonal effects on dispersal state or interaction between body mass and season could be detected (Fig. 3), but this result should be regarded as preliminary because of low sample size for the post-dispersal phase during TRS. The highly significant intercept of our calculated model ( $p=0.001$, Tab. 1 ) indicated that our explanatory factors were not sufficient to capture most of the variation in transition probabilities. These results remained the same when we exchanged body mass with $B C$ in our model (estimate $=3.843, S E=1.50 z=2.55, p=0.01$, Tab. 2).

\subsection{Relationship between dispersal distances, sex and body mass}

Analyses of the relationship between body mass and dispersal distance were based on data from 26 males weighted in the pre-dispersal phase and 51 males in the post-dispersal phase. Observed dispersal distances ranged between $150 \mathrm{~m}$ and 1,010m. Body mass had no effect on dispersal distance (Tab. 3, Fig. 5, pre-dispersal: $p=0.99$; post-dispersal: $p=0.58$ ). This relationship did not change, when we used BC as predictor (Tab. 3, Fig. 5, $n_{\text {pre-dispersal }}=19$, 
CHAPTER 2: PROXIMATE MECHANISMS OF DISPERSAL

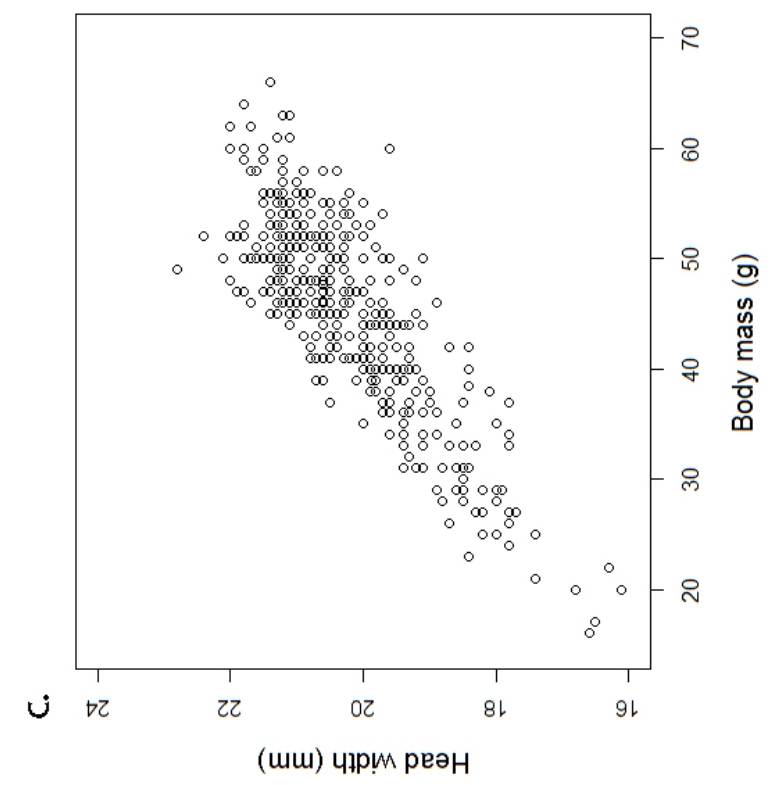

อิ

ำㅇ

总 结

음

뇐

¿

丞

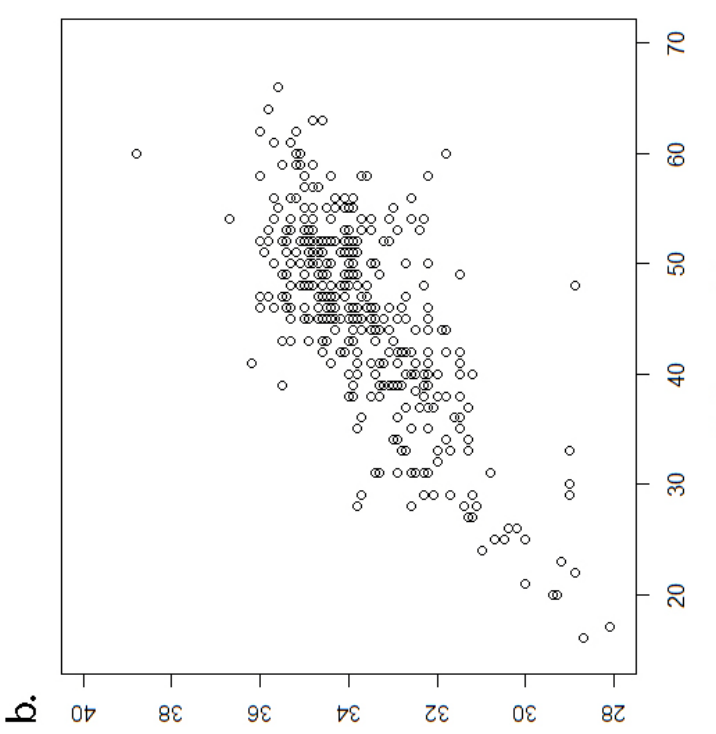

(uш) чฺбиәә реән

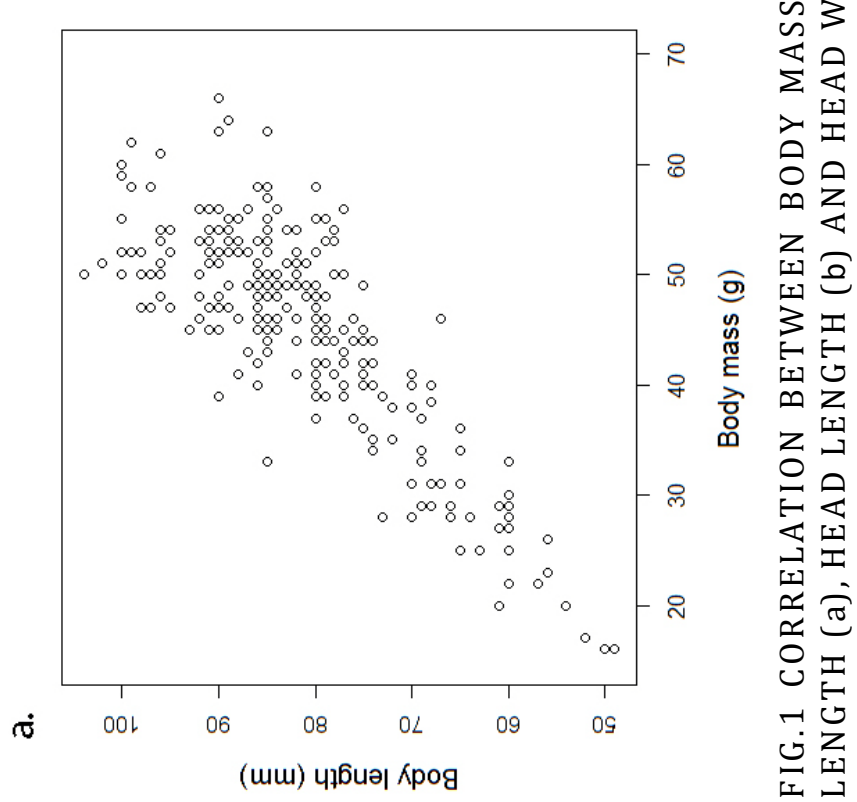




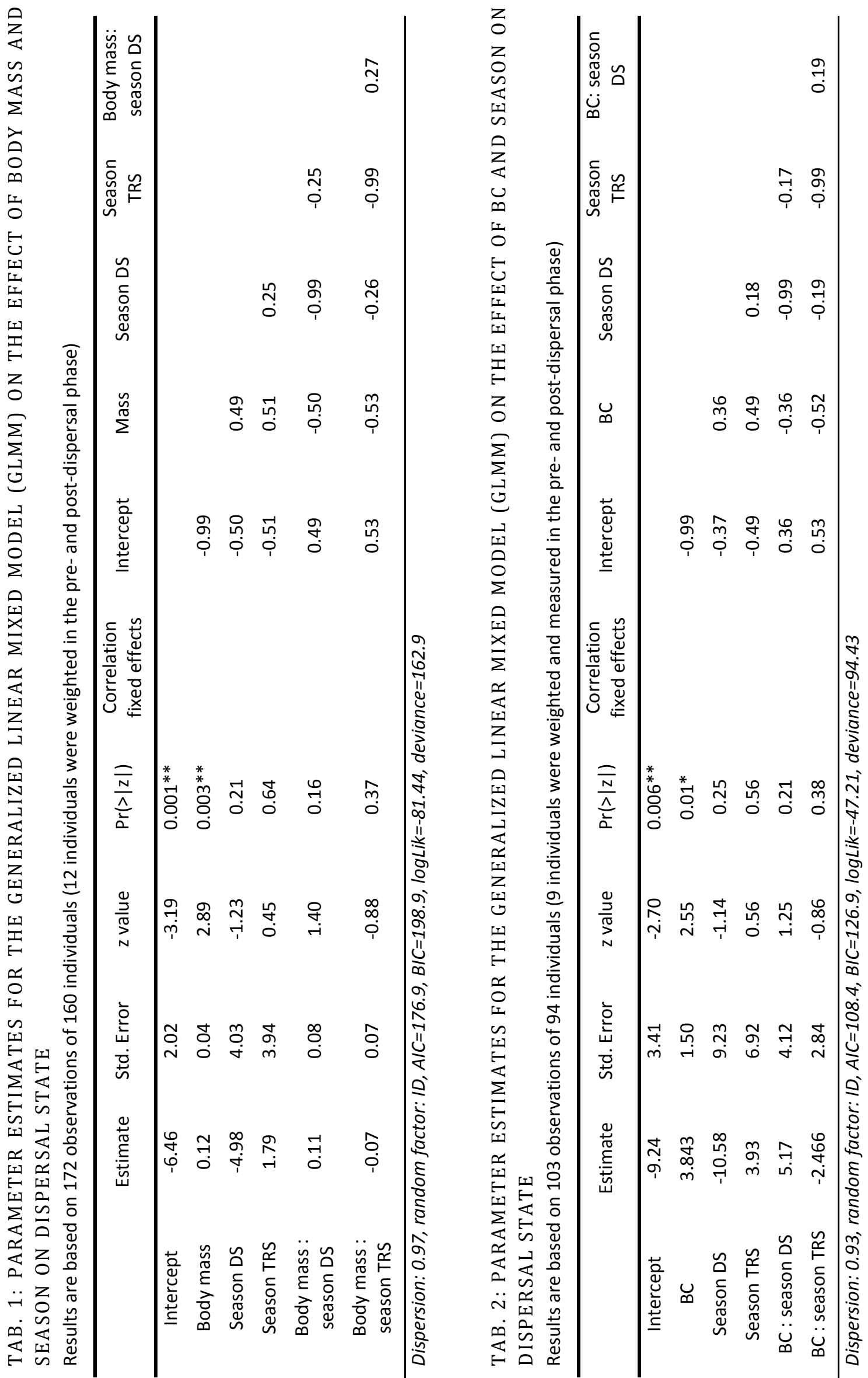


CHAPTER 2: PROXIMATE MECHANISMS OF DISPERSAL

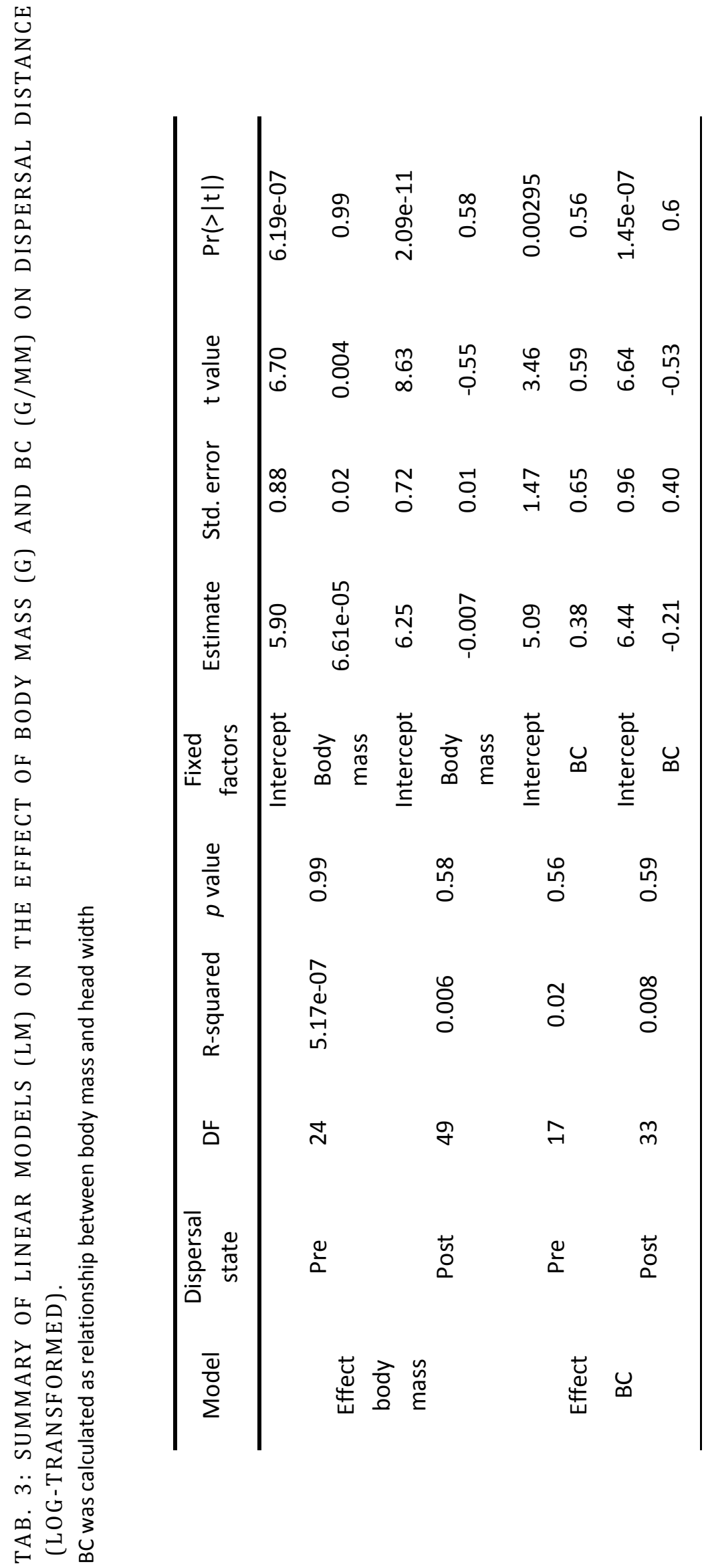




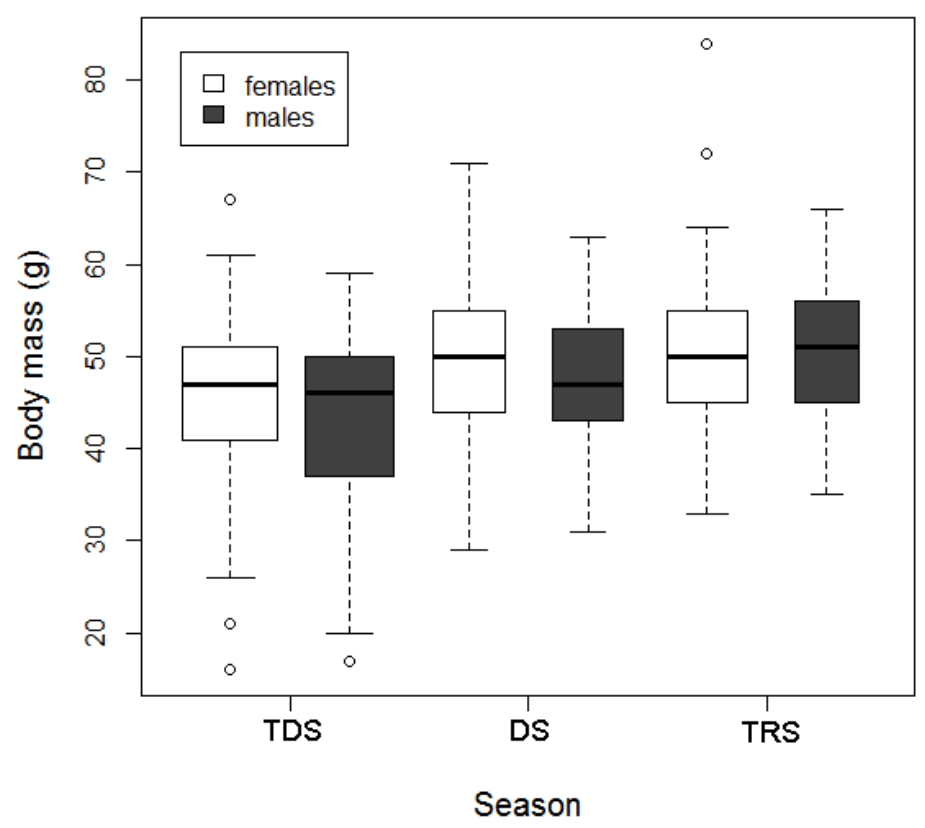

FIG.2 AVERAGE BODY MASS OF SUBADULT MALES AND FEMALES MEASURED DURING THE THREE DIFFERENTIATED SEASONS

a.

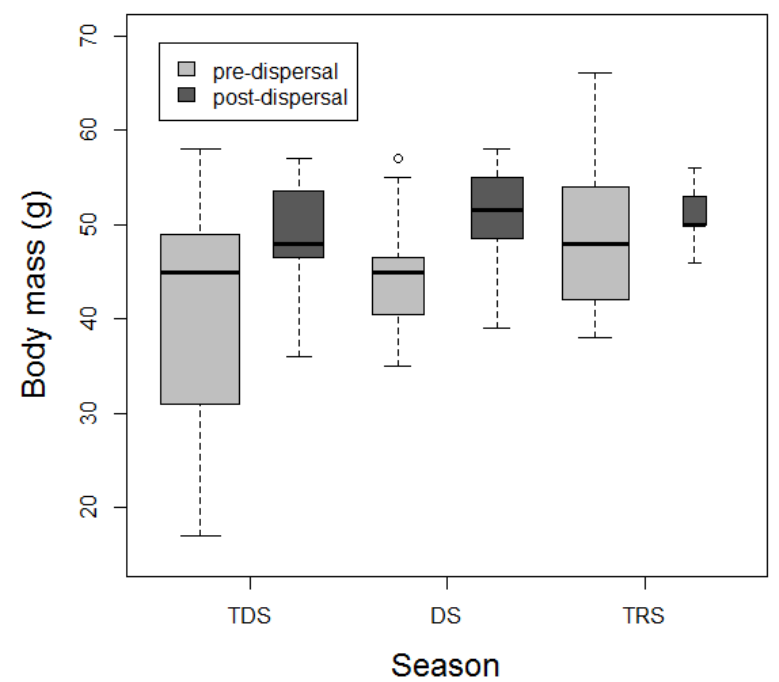

b.

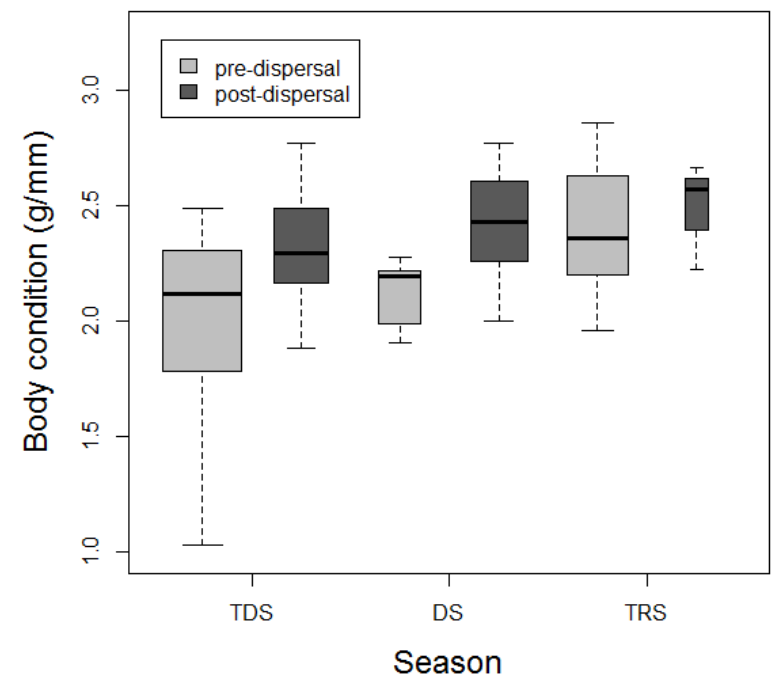

FIG.3: BODY MASS (a) AND BODY CONDITION (b) OF NOT (YET) DISPERSED (PREDISPERSAL) AND DISPERSED (POST-DISPERSAL) MALES MEASURED DURING THE THREE DIFFERENTIATED SEASONS

A maximum of one data point per state per individual was included (see methods). Sample sizes of body mass for the pre-dispersal state were as follows: $n_{\mathrm{TDS}}=52, n_{\mathrm{TDS}}=28, n_{\mathrm{TDS}}=40$. For the post-dispersal state we included the following number of data points: $n_{\mathrm{TDS}}=23, n_{\mathrm{TDS}}=24, n_{\mathrm{TDS}}=5$. Sample sizes of pre-dispersal BC were $n_{\mathrm{TDS}}=35$, $n_{\mathrm{TDS}}=10, n_{\mathrm{TDS}}=21$. For post-dispersal BC we included the following number of data points: $n_{\mathrm{TDS}}=16, n_{\mathrm{TDS}}=18$, $n_{\mathrm{TDS}}=3$ 
a

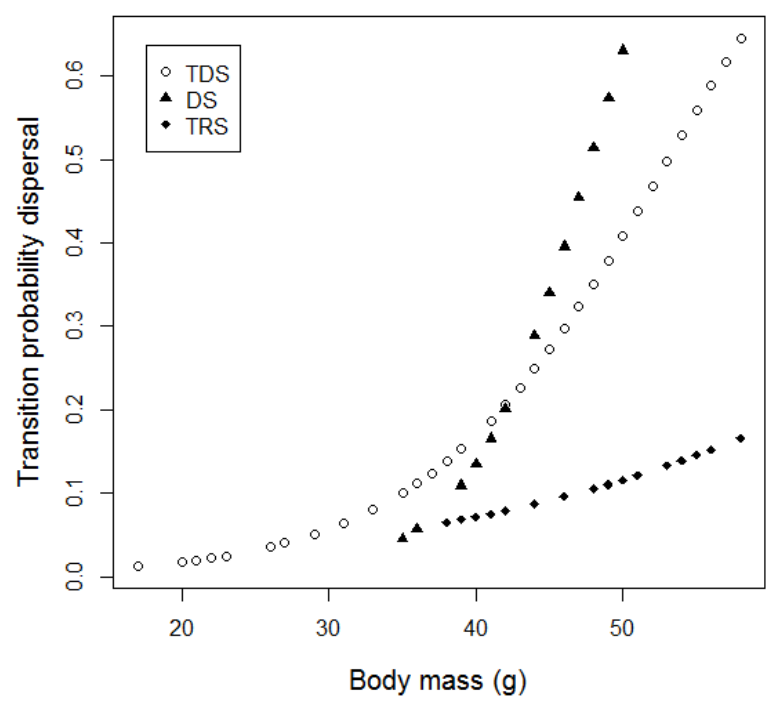

b.

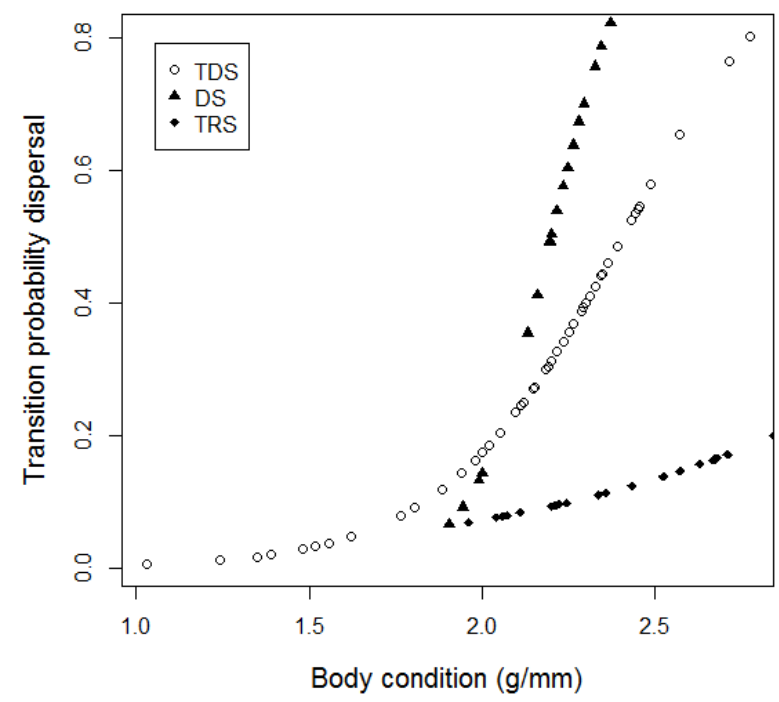

FIG.4: FITTED TRANSITION PROBABILTY FROM THE PRE- TO THE POST-DISPERSAL STATE DEPENDENT ON BODY MASS (a) OR BC (b) INTERACTING WITH SEASON

A maximum of one data point per state per individual was included (see methods). Sample sizes of body mass for the pre-dispersal state were as follows: $n_{\mathrm{TDS}}=52, n_{\mathrm{TDS}}=28, n_{\mathrm{TDS}}=40$. For the post-dispersal state we included the following number of data points: $n_{\mathrm{TDS}}=23, n_{\mathrm{TDS}}=24, n_{\mathrm{TDS}}=5$. Sample sizes of pre-dispersal BC were $n_{\mathrm{TDS}}=35$, $n_{\mathrm{TDS}}=10, n_{\mathrm{TDS}}=21$. For post-dispersal $\mathrm{BC}$ we included the following number of data points: $n_{\mathrm{TDS}}=16, n_{\mathrm{TDS}}=18$, $n_{\mathrm{TDS}}=3$

$\left.p=0.56 ; n_{\text {post-dispersal }}=35, p=0.6\right)$. Seasonal effects remained unevaluated because of the low sample size for DS.

\section{DISCUSSION}

\subsection{Influence of body mass on dispersal probability}

Species-specific mechanisms of timing of dispersal remain poorly understood. In male gray mouse lemurs, the probability to be a disperser or not (yet) is related to body mass. Body mass was in turn strongly correlated with morphological measures of subadults and therefore reflected differences in developmental state rather than in BC. Developmental state and reaching a critical body size therefore present a first hurdle for dispersal in gray mouse lemurs. A study on captive grey mouse lemurs indicated that juveniles grow extremely fast and that they can reach a body mass of almost $30 \mathrm{~g}$ within three weeks (Perret 1990). The fast growth is probably necessary for subadults to be able to survive and feed independently of mothers before the onset of the dry season. Most likely, with the minimum required body size for dispersal individuals reach a level of competence that, on the one hand, allows them to survive independently of the protection of the family group (Alberts 
and Altmann 1995b) and, on the other hand, reduces risk of dispersal for survival caused by high travel activity and/or unfamiliarity with a novel environment (Belthoff and Dufty 1998; Clobert et al. 2009). Since we found no sexual dimorphism in subadults, males neither pursued a different growth strategy, nor did they suffer from pronounced changes in development through dispersal. In contrast, adult gray mouse lemurs do show a seasonally fluctuating dimorphism (Schmid and Kappeler 1998). Adult females are usually heavier than adult males at the onset of the dry season, because many of them enter long periods of inactivity at our study site, whereas adult males usually gain weight towards the mating season to increase reserve assets for high travel activity and competition with other males during the mating season (Schmid and Kappeler 1998; Schmid 1999; Eberle and Kappeler 2004b). Probably, growth is the main priority during the development of subadult gray mouse lemurs, whereas accumulation of energy storages starts when the bigger part of growth is already accomplished.

One important source of differences in developmental state could be variation in birth date. Matings are distributed over a 4-weeks period and females may enter a second estrus under some conditions (Wrogemann et al. 2001; Eberle and Kappeler 2004b). Thus, individual birth dates can vary substantially despite seasonal breeding. As a consequence, some juveniles might face more unfavorable conditions for growth and, hence, delay development (Castanet et al. 2004). Other sources of differential development include the loss of mothers (see Eberle and Kappeler 2006) or differences in habitat quality between patches or years.

Our data indicate that gray mouse lemur males need to reach a critical size before dispersal is induced. Similar observations have been made for banner-tailed kangaroo rats (Edelman 2011) and ground squirrels (Nunes and Holekamp 1996; Nunes et al. 1998). In both species, food supplementation let to earlier dispersal, and for ground squirrels high body fat was a critical prerequisite to survive following overwintering. In marsh tits (Nilsson and Smith 1985; 1988), crested tits (Lens and Dhondt 1994), red squirrels (Wauters et al. 1993), common shrews (Hanski et al. 1991) and freshwater crocodiles (Tucker et al. 1998), higher body mass has been equated with higher competitive abilities and for the first three species led to changes in dispersal timing. For gray mouse lemurs, once this critical threshold of development is reached, other factors determine the exact timing of dispersal as indicated by the high significance of the intercept of our model (Tab. 1, 2). The nature and relative 
importance of these factors still needs to be evaluated. Hormonal changes (Dufty and Belthoff 2001; Dufty et al. 2002), resource availability (Dammhahn and Kappeler 2008b), population density and personality differences (Cote et al. 2012; Dammhahn 2012) present promising possibilities for further investigations. Examining these factors could help to elucidate why some males dispersed as soon as they reached the critical body mass whereas others remained philopatric despite a body mass well beyond this threshold.

a.

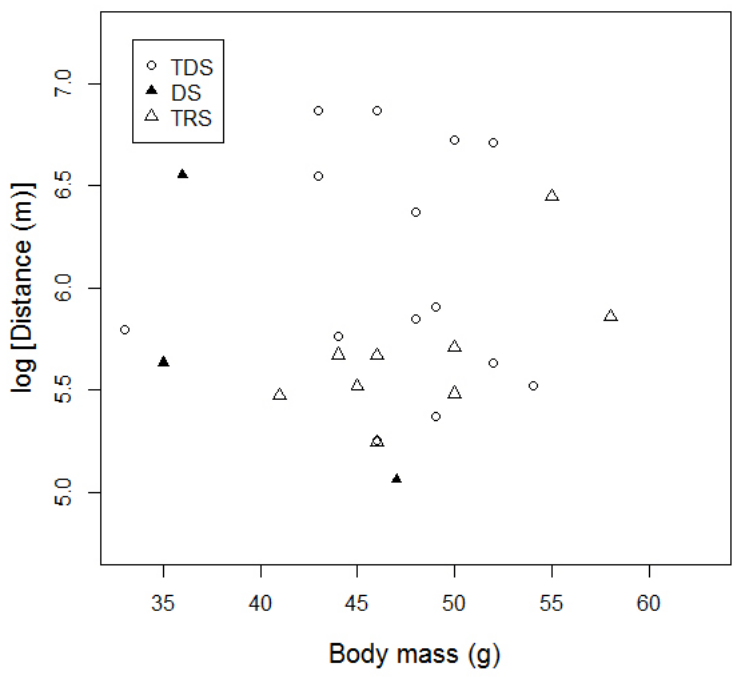

c.

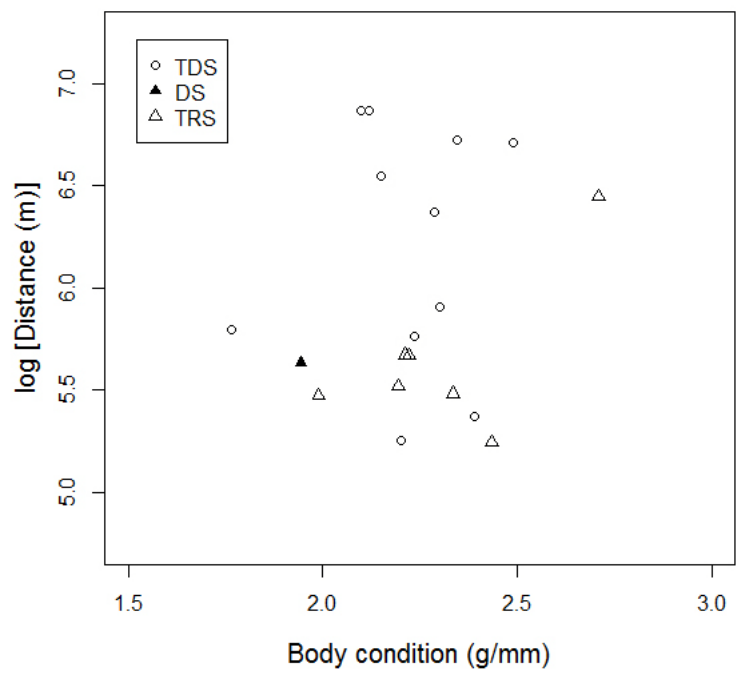

b.

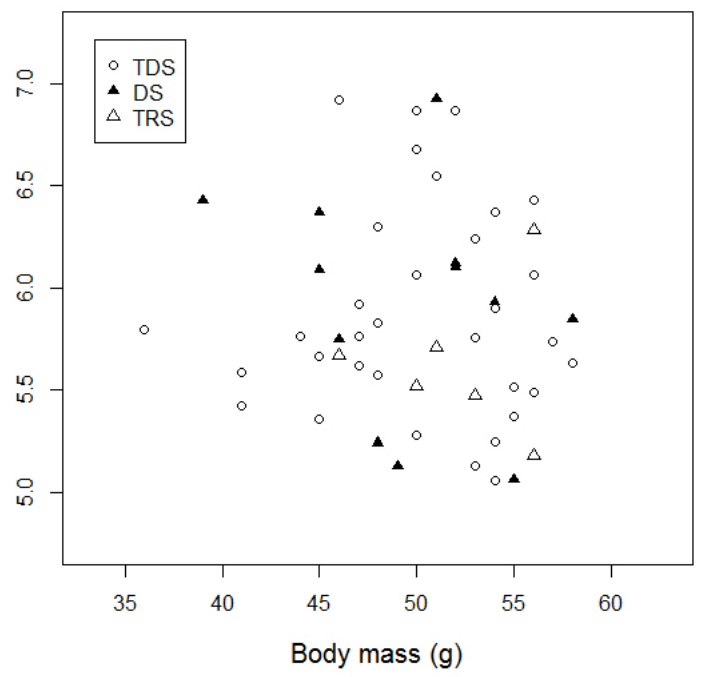

d.

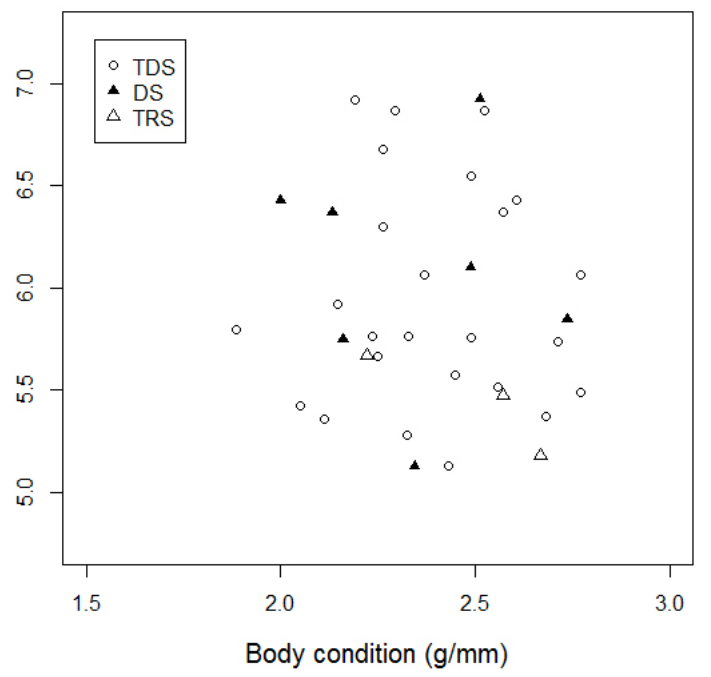

FIG.5: RELATIONSHIP BETWEEN BODY MASS, BC AND DISPERSAL DISTANCES FOR DATA COLLECTED FOR INDIVIDUALS IN THE PRE- (a, c) AND THE POST-DISPERSAL STATE $(b, d)$

Different symbols indicate dispersal events from the three differentiated time periods. BC depicts the ratio of body mass to head width 
Variation in timing of dispersal could have various consequences for males. Possibly, males face a trade-off between optimal size and condition and familiarity with a new area and the quality of a chosen area (Belthoff and Dufty 1998; Edelman 2011). We would assume that possible advantages of early dispersal include greater familiarity with a new home range during the dry season and/or the mating season. The alternative strategy would be to remain in the natal area and to profit from increased growth and competitive abilities in comparison to early dispersers, but risk a disadvantage in locating unrelated females during the mating season (Eberle and Kappeler 2004b). A comparable trade-off between condition and timing of dispersal has been observed for male banner-tailed kangaroo rats (Edelman 2011). For them, remaining at their natal site beyond a critical size or condition would subsequently increase the competition for mounds, a crucial resource for this species. Whether the threshold of body mass for dispersal is fixed in the course of the year irrespective of changes in food availability still needs to be evaluated. Also, the existence of variation of the threshold between years remains to be evaluated. If the threshold changes between time periods of different food abundance, this information would indicate whether dispersal has a negative effect on BC (Nunes et al. 1998). The transition probabilities indicated that the influence of body mass on dispersal state was much lower during TDS, when food availability increases again. Seasonal effects could pronounce or reduce the effect of physical condition. In summary, gray mouse lemurs seem to be sensitive towards internal (phenotypic) and external cues and match their dispersal behavior to prevailing conditions, which most likely improves their chances of successful dispersal, but the exact mechanism and interactions between internal and external cues of dispersal still need further investigations.

\subsection{Relationship between dispersal distances and body mass}

The assumption that a trade-off exists between dispersal distance and physical condition was not confirmed in this study. There were no effects of body mass and BC on dispersal distance of male gray mouse. There are several possible explanations for these results. One explanation is that realized dispersal distances did not present a challenge for individuals since dispersal distances of up to $1 \mathrm{~km}$ did not exceed the average nightly travel distances (Dammhahn and Kappeler in press). Moreover, observations of dispersing gray mouse lemurs indicated that individuals regularly commute between old and new home ranges 
during the transfer phase (Chapter 1). Since males seem to move back and forth quite easily, unfamiliarity with the novel habitat seems to inflict more costs in terms of energy than the distance between old and new habitat.

Another reason for the lack of a strong effect of body mass and condition on dispersal distance could originate from the uneven spatial distribution of gray mouse lemur populations (Fredsted et al. 2004). Predetermined dispersal distances could strongly reduce the frequency of successful dispersal between population patches and thus gene flow. The stereotypic strategy of gray mouse lemur males to disperse in directed movements with fixed individual destination might also require more flexibility in realized dispersal distances (Chapter 1). Spatial knowledge of subadults beyond their natal home range seems to be rather limited and exploration efforts during dispersal cover a very restricted area (Lührs et al. 2009; Chapter 1). Possibly, when individuals have decided on the direction of their individual dispersal trajectories, dispersal distance is dependent on the gradient of habitat quality individuals experience on this trajectory. Dispersal distance would only have an upper limit, up to which animals are able to trace their steps back to their natal area. Studies on mechanisms of habitat selection could be insightful in terms of potential determinants of dispersal distance. Beside habitat quality, these approaches should also incorporate social components like conspecific attraction to see how population densities and sex-ratios influence settling probability of males (Stamps 2001). Genetic influences on exploratory behavior represent another important factor to incorporate (Selonen and Hanski 2010).

\section{CONCLUSIONS}

Behavioral flexibility in the timing of dispersal allows animals to match their behavior to current internal and external conditions to reduce dispersal related mortality risks like starvation. However, this flexibility can incorporate a rather invariable threshold of minimum required maturity and physical condition. Despite the deterministic influence of development on the dispersal decision, dispersal distances seem to be influenced by other factors. Broadening our understanding about habitat selection mechanisms will be crucial for the understanding of why individuals disperse over a given distance. Finally, we would like to raise a note of caution concerning the interpretation of individual dispersal events, which are often interpreted in terms of ultimate explanations without controlling for (obvious) proximate causation. For example, dispersal during mating periods does not 
necessarily represent a response to potential inbreeding risk, but could be a result of illmatched conditions, which delayed dispersal until this period. Especially, when differences in timing of dispersal exist in a species with otherwise rigid sex-specific dispersal propensities, it seems more apt to look first at differences between individuals at the proximate level.

\section{ACKNOWLEDGEMENTS}

We acknowledge the authorization of this study by the Commission Tripartite and the CAFF of the Direction des Eaux et Forêts, the CNFEREF. All research reported here is in compliance with animal care regulations and applicable national laws of Germany and Madagascar. The appropriate Animal Use and Care committees of Germany (Bundesministerium für Naturschutz, BfN) and Madagascar (Ministère de l'Environnement et des Eaux et Forêts, MINEEF) approved all research protocols. The CRCESP and the Christian-Vogel-Fonds (GFP) provided financial support. We thank Dr. Rodin Rasoloarison, Léonard Razafimanantsoa, Tiana Andrianjanahary, Bruno Tsiveramana, Remy de Ampataka and the Equipe Kirindy for support and assistance in the field. 
CHAPTER 2: PROXIMATE MECHANISMS OF DISPERSAL 
Chapter 3: SeX-specific Behavioural Differences

On the application of mixed hidden Markov models to multiple behavioural time series

with Peter M. Kappeler and Roland Langrock 


\section{ABSTRACT}

Analysing behavioural sequences and quantifying the likelihood of occurrences of different behaviours is a difficult task as motivational states are not observable. Furthermore, it is ecologically highly relevant and yet more complicated to scale an appropriate model for one individual up to the population level. In this manuscript (mixed) hidden Markov models (HMMs) are used to model the feeding behaviour of 54 subadult grey mouse lemurs (Microcebus murinus), small nocturnal primates endemic to Madagascar that forage solitarily. Our primary aim is to introduce ecologists and other users to various HMM methods, many of which have been developed only recently, and which in this form have not previously been synthesized in the ecological literature. Our specific application of mixed HMMs aims at gaining a better understanding of mouse lemur behaviour, in particular concerning sex-specific differences. The model we consider incorporates random effects for accommodating heterogeneity across animals, i.e. accounts for different personalities of the animals. Additional subject- and time-specific covariates in the model describe the influence of sex, body mass and time of night.

Keywords: behavioural analysis; maximum likelihood; motivational states; random effects; state-space model; subject-specific covariate

\section{INTRODUCTION}

When analysing how selection has shaped behaviours we observe today, it is usually assumed that an animal's decisions approximate an optimal solution based on the amount of information available to the individual (McNamara and Houston 2009). Individuals are believed to balance trade-offs based on profitability and availability, and behavioural decisions of past generations are assumed to have been selected for a maximal contribution to the phenotypic fitness of the animals, so that current decisions can therefore be regarded as adaptations (MacArthur and Pianka 1966; Charnov 1976; Caraco 1980; McNamara and Houston 2009). But each member of a species is distinct from its conspecifics. Some of these differences between individuals may be temporary and affect state variables, e.g. hunger, thirst, fear, whereas others concern fixed, long-term or slowly changing individual parameters, e.g. size, sex, age, degree of maturity, reproductive states and personality traits 
(Houston and McNamara 1999). The attribution of factors into these classes might differ between studies since their persistence/continuation might also depend on the time scale of a study (Yackulic et al. 2011). Nevertheless, both types influence the so-called 'motivational state'. This term can be defined as the motivation of an individual generated by physiological and perceptual states (McFarland 1999). The motivational state influences the likelihood of an occurrence of behaviour.

Determining the motivational state of free-ranging animals is a complex task. It is generally accepted that individuals have mechanisms to monitor their internal state (McFarland 1999). The problem for behavioural ecologists is that the current motivational state of an individual includes many hidden aspects like physiological states (e.g. hormone and metabolite levels or protein and lipid stores), but also externally based motivational aspects (e.g. perceived predation risk) (Whitten et al. 1998; Kyriazakis et al. 1999; Winnie Jr. et al. 2006). Estimates of the motivational state could be derived from physiological measures like hormone profiles (Whitten et al. 1998), or from behaviours that are specific for a certain context, e.g. courtship behaviour (Riters et al. 2000). But most often, motivational states or state changes remain a black box. The link between the typically unobserved motivational state and the actually observed behaviour is often not one-to-one; e.g. a hungry individual might have problems finding appropriate food, or it might be distracted by a perceived high predation risk, and thus not feed (McFarland 1999). Some early attempts to model behavioural sequences - before the models we consider here have been developed - used Markov chains to explain the observed behaviour, thus not explicitly modelling the motivational component (Morgan 1976). Transitions between internal states, such as moving/pausing or hungry/satiated, can typically only be inferred posthoc, e.g. through gaps between feeding bouts. Thereby information about the actual behavioural process is lost because data are often simplified and/or converted to proportions, and because patterns are evaluated using statistical tests only. Moreover, the occurrence of motivational states for a single animal will not be independent over time (Houston and McNamara 1999), a fact that is often implicitly accepted.

Models integrating a link between motivational state and behaviour are relatively sparse. One of their requirements is that the model includes a probabilistic relationship between the action chosen and the animal's state (Houston and McNamara 1999). Dependent mixture models such as hidden Markov models (HMMs) incorporate the 
presence of these underlying motivational states, as well as their autocorrelation, and facilitate their inference (MacDonald and Raubenheimer 1995; Frühwirth-Schnatter 2006; Zucchini et al. 2008; Patterson et al. 2009; Pedersen et al. 2011). The different components of the mixture can conveniently be interpreted as being associated with the different motivational states of the animal. HMMs are relatively simple stochastic models that nevertheless exhibit immense flexibility; besides ecology they have proved useful in fields such as speech recognition (Rabiner 1989) (for which purpose they were originally developed), finance (Banachewicz and Vaart 2008; Langrock et al. 2012), economics (Hamilton 1989), biology in general (Hamilton 1989; Durbin et al. 1998), computer vision (Vogler and Metaxas 1997) and climatology (Zucchini and MacDonald 2009). Besides many other convenient features - such as the straightforward treatment of missing data - HMMs also facilitate the inference about underlying motivational states, enabling us to predict the most likely motivational state sequence (Zucchini et al. 2008), a feature that is not exploited in the current analysis, however. Classical likelihood-based inference for HMMs is convenient and efficient, it is thus not necessary - albeit possible (Scott 2002) - to apply Bayesian methods, which despite growing popularity in the ecological literature (Clark 2005; King et al. 2010) are presumably less accessible to practitioners in the case of HMMs. In a Bayesian approach, it is in particular difficult to estimate the number of states of an HMM, and the issue of the so-called label switching needs to be addressed (Scott 2002).

HMMs have precisely the same dependence structure as state-space models (SSMs); the former assume a finite number of states, while the state space in the latter may be infinite. In recent years, SSMs have become increasingly popular tools for modelling animal behaviour, in particular animal movement (Buckland et al. 2004; Royer et al. 2005; Jonsen et al. 2006; Patterson et al. 2008; Breed et al. 2009). However, the likelihood of SSMs with infinite state space involves a multiple integral that, in general, cannot be evaluated directly. In particular, nonlinear and non-Gaussian SSMs, to which the Kalman filter is not directly applicable, are rather difficult to fit. The literature offers a variety of possible methods for estimating the parameters of such models (Melino and Turnbull 1990; Welch and Bishop 1995; Durbin and Koopman 1997; Langrock 2011). Given the difficulties involved in fitting SSMs, it sometimes may be more convenient to resort to the less flexible special case HMMs, if appropriate (Patterson et al. 2009). In some applications, the assumption of a finite number of (motivational) states can be perfectly reasonable. However, observation errors, 
e.g. caused by measurement inaccuracies in animal movement paths, can more easily be accommodated in the SSM framework (Patterson et al. 2008).

Scaling individual models up to the population level is an issue of great ecological relevance. There are several different ways in which basic HMMs can be extended to deal with multiple time series (see the discussion in $\$ 2.3$ below). In this paper, we follow suggestions of Zucchini et al. (2008) and propose a model that incorporates both subjectspecific covariates and random effects, combining - to some extent - the benefits of both approaches, which in this form to the best of our knowledge has not been done before in the ecological literature. (Zucchini et al. (2008) incorporate one random effect, but no covariates in their model; ecological applications of HMMs that involve covariates, but no random effects, are given, for example, in Patterson et al. (2009) and Morales et al. (Morales et al. 2004), although in the latter case, the model is not explicitly referred to as an HMM.) Our model belongs to the class of mixed HMMs (Altman 2007).

The primary aim of this paper is to provide ecologists and other practitioners with a comprehensible introduction to mixed HMMs, and to discuss their potential in statistical ecology, particularly concerning analyses of multiple series. The explanations of the basic ideas and the associated methodology are given for one specific application, rather than in a more general manner. We chose this strategy for the presentation as the given application provides a very convenient means of introducing and illustrating the methods, and as for HMMs it is usually straightforward to transfer the basic ideas to other applications. On the other hand, the application given here is interesting in its own right, and we thus describe it in much detail. More specifically, we use a mixed HMM to model the feeding behaviour of a population of subadult (less than 1 year) grey mouse lemurs. The grey mouse lemur (Microcebus murinus) is a small (60 g), monomorphic, nocturnal, solitary primate and can be found from the dry deciduous forests in western and north-western Madagascar to the evergreen littoral rain forests and spiny forests in the south of the island (Rasoloarison et al. 2000; Kappeler and Rasoloarison 2003; Mittermeier et al. 2010). They feed opportunistically on insects, small vertebrates, fruits, gum and insect secretions, and the composition of their diet varies with season (Dammhahn and Kappeler 2008a; b; 2010). In contrast to adult individuals, most of the subadult individuals do not engage in longer phases of inactivity, but remain active during the dry season characterizing western Madagascar (Schmid 1999). Another distinctive feature of subadults from the male perspective is that subadult males 
separate from their families to disperse (Eberle and Kappeler 2002; Radespiel et al. 2003; Fredsted et al. 2005). The solitary lifestyle of mouse lemurs (individual foraging decisions are not dependent on conspecifics as in group-living species), as well as general differences in the life-history strategies between males and females, makes them a good case for evaluating a mixed HMM for behavioural sequences.

\section{MATERIAL AND METHODS}

\subsection{Study site, animals and data description}

Behavioural data were collected for subadult grey mouse lemurs of a study population situated within a 12,500 ha forestry concession of the Centre National de Formation, d'Etude et de Recherche en Environnement et Foresterie (CNFEREF) de Morondava in Kirindy Forest, $60 \mathrm{~km}$ northeast of Morondava in western Madagascar (448390 E, 208030S; Sorg et al. 2003). The region is characterized by pronounced seasonality with a single rainy season lasting from December to March. The study took place in a 60 ha area, locally known as CS7 (for details see Eberle and Kappeler 2002). In this area, we captured subadult M. murinus and equipped them with radio collars for radio tracking (Holohil Systems Ltd. BD-2C; $1.8 \mathrm{~g}$ ).

We collected behavioural data for 16 subadult females and 38 subadult males between 2008 and 2010. Sampling periods lasted from April to May in 2008, May to October in 2009 and May to July in 2010. Four individuals were observed per night over $40 \mathrm{~min}$ periods between 18.00 and $24.00 \mathrm{~h}$ in randomly changing combinations and order. Feeding data of focal animals were collected cumulatively for observation intervals of 1 min (Martin and Bateson 1993). When the individual was not visible, these minutes were recorded as missing data (NA). About $500 \mathrm{~h}$ of focal observations were included in the present analyses. The numbers of available time series differ between focal animals because of predation events, non-functioning radio collars and differences in the length of total observation periods per year. Therefore, the dataset was heterogeneous. Numbers of available time series of feeding behaviour per individual ranged between 1 to 26 (mean=9). Body mass of individuals ranged between 33 to $59 \mathrm{~g}$ (mean=48 g) with a mean body mass of $49 \mathrm{~g}$ for females and $47 \mathrm{~g}$ for males. 


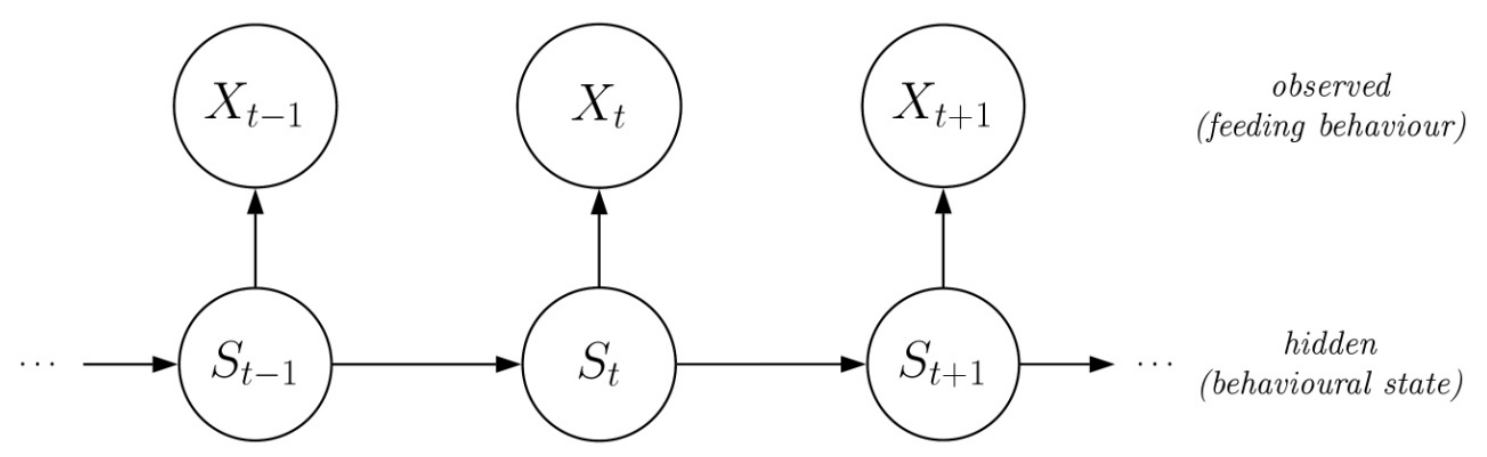

FIG. 1: DEPENDENCE STRUCTURE OF AN HMM

\subsection{Stochastic model for a single animal}

For illustration purposes, we start by considering a relatively simple HMM for a single animal before we move on to the more challenging population models in $§ 2.3$. Figure 1 represents the (dependence) structure of a basic HMM. The state process cannot be observed (it is hidden). In our application, it can be interpreted as generating the motivational states of the observed animal; $S_{t}$ then is associated with the motivational state of the animal at time $t$. We model lega by a Markov chain, in particular assuming that the distribution of St is completely determined by the motivational state the animal is in at time $t-1$ :

$$
\begin{aligned}
\mathbb{P}\left(S_{t}\right. & \left.=s_{t} \mid S_{t-1}=s_{t-1}, S_{t-2}=s_{t-2}, \ldots\right) \\
& =P\left(S_{t}=s_{t} \mid S_{t-1}=s_{t-1}\right) .
\end{aligned}
$$

The Markov chain thus is of first order. We fit models that involve two different motivational states. In the context of feeding behaviour, it seems convenient to label the two states by 'satiated' (state 1) and 'hungry' (state 2), respectively, though they do not necessarily correspond to the accepted meanings of those terms. Irrespective of how the motivational states are defined, most importantly, their delineation will provide us with an objective measure of the general motivational state, allowing us to explore what factors influence the transitions between activities (feeding / non-feeding) of the observed individuals. If the animal is in state $i$ at time $t$, the probability of it being in state $j$ at time $t+1$ is:

$$
\left.\gamma_{i j}^{(t)}=\mathbb{P}\left(S_{t+1}=j\right) \mid S_{t}=i\right) \text {. }
$$

(Example: $\gamma_{12}^{(t)}$ is the probability that the animal will be hungry at time $t+1$, given that it is satiated at time $t$.) 
As there is no a priori reason to assume that the occurrences of motivational states are homogeneously distributed over the night, we model the transition probabilities between motivational states as a function of time:

$$
\operatorname{logit}\left(\gamma_{i i}^{(t)}\right)=\beta_{0, i}+\beta_{1, i} t, \quad i=1,2, \quad \mathrm{t}=0,1,2, \ldots
$$

The logit link ensures that $\gamma_{i i}^{(t)}$ is in $[0,1]$. It is straightforward to make this generalized linear model for $\gamma_{i i}^{(t)}$ more flexible by considering quadratic or even cubic functions of the covariate $t$. However, for simplicity, and as we are primarily interested in whether there is any trend at all, we used a simple linear predictor here. From state $i$ the process can only switch to state $\mathrm{j}$ or remain in state $\mathrm{i}$, and so $\gamma_{i j}^{(t)}=1-\gamma_{i i}^{(t)}$ for $j \neq i$. Each integer time $t$ refers to $1 \mathrm{~min}$. At time $t=0$ - corresponding to $18.00 \mathrm{~h}$ in our application - the state is selected by an initial distribution

$$
\delta=\left(\mathbb{P}\left(S_{t}=1\right), \mathbb{P}\left(S_{t}=2\right)\right)
$$

The non-observable motivational states determine the distributions associated with the observed behaviour. We observe the behaviour $X_{t}$, where $X_{t}=0$ if the animal does not feed at time $t$, and $X_{t}=1$ if the animal does feed at time $t$. The model assumes that, given the motivational state at time $t$, the distribution of the behaviour $X_{t}$ is independent of all previous states and observations. More precisely, we assume $X_{t}$ to follow a Bernoulli distribution (i.e. a binomial distribution of size $n=1$ ), where the parameter is driven by the (motivational) state the animal is in at time $t$ :

$$
P\left(X_{t}=1 \mid S_{t}=k\right)=\pi_{k}, k=1,2 .
$$

As $\pi_{2}$ is associated with the animal being in the 'hungry' state, it will typically be relatively large, while $\pi_{1}$ (feeding probability when satiated) can be expected to be close to 0 .

There are in total seven parameters to be estimated (one for the initial distribution, four for the transition probabilities and two for the state-dependent process). The model fitting exercise is usually carried out using numerical maximization of the likelihood function, which is given by a closed-form matrix product:

$$
\begin{aligned}
\mathcal{L}=\delta & P_{\pi}\left(x_{1}\right) \Gamma^{(1)} P_{\pi}\left(x_{2}\right) \Gamma^{(2)} \cdot \ldots \\
& \cdot P_{\pi}\left(x_{T-1}\right) \Gamma^{(T-1)} P_{\pi}\left(x_{T}\right) 1^{t} .
\end{aligned}
$$


Here $1 \in \mathbb{R}^{2}$ is a row vector of ones, $T$ denotes the number of observations, $\Gamma^{(t)}$ is the transition probability matrix at time $t$,

$$
\Gamma^{(t)}=\left[\begin{array}{ll}
\gamma_{11}^{(t)} & \gamma_{12}^{(t)} \\
\gamma_{21}^{(t)} & \gamma_{22}^{(t)}
\end{array}\right] .
$$

and $P_{\pi}\left(\chi_{t}\right)=\operatorname{diag}\left(\pi_{1}^{x_{t}}\left(1-\pi_{1}\right)^{1-x_{t}}, \pi_{2}^{x_{t}}\left(1-\pi_{2}\right)^{1-x_{t}}\right)$; for missing observations, this is replaced by the $2 \times 2$ - identity matrix. For more details on the derivation and the numerical maximization of an HMM likelihood, we refer to chapters 2 and 3 in Zucchini \& MacDonald (2009). Alternatively, one can apply the expectationmaximization (EM) algorithm (Welch 2003). In $\S 2.3$, we extend this basic HMM to capture the heterogeneity of multiple time series, associated with a population of grey mouse lemurs.

\subsection{Stochastic model for a population of individuals}

The class of HMMs provides several different strategies for dealing with populations of time series. For instance, one might impose the very restrictive assumption that the parameter set is common to all subjects. This strategy neglects any possible heterogeneity across subjects: two individuals, regardless of their sex, age, mass, personality, etc., would be assumed to act according to the same (stochastic) principles. Another extreme strategy assumes that each of the parameters is subject-specific, i.e. that each subject has its own set of parameters. This approach involves a significantly larger number of parameters and generally ad hoc comparisons between individuals. In between these two extreme options lies the compromise of assuming that some parameters - e.g. those determining the statedependent process - are common to all subjects, while the others are subject-specific. An important special case of the latter is to assume that the subject-specific parameters - the random effects - are drawn from a common distribution. This approach substantially reduces the number of parameters to be estimated. HMMs incorporating random effects were considered, for example by (Humphreys 1998; Seltman 2002; Zucchini et al. 2008). Random effects can be understood as explaining the individuality (or personality) of the different animals. Unfortunately, their implementation is very demanding in terms of computational effort (Altman 2007). A computationally less expensive way of accounting for possible heterogeneity across subjects is to incorporate subject-specific covariates in the model (MacDonald and Zucchini 1997; Wang and Puterman 2001; Bartolucci et al. 2009). Such a 
model may explain heterogeneity across subjects, but it requires that suitable covariates are available. In the given application, we will consider sex and body mass of individuals, covariates that may help to explain individual differences, but only to a limited extent.We will thus also incorporate randomeffects in the model, in this way combining the benefits of both approaches. More precisely, for animal $m, m=1, \ldots, M$, we assume the motivational state-transition probabilities at time $t$ to be determined by:

$$
\operatorname{logit}\left(\gamma_{i i}^{(t, m)}=\beta_{0, i}+\beta_{1, i} t+\beta_{2, i} \operatorname{sex}^{(m)}+\beta_{3, i} \operatorname{mass}^{(m)}\right.
$$

$(i=1,2, t=0,1,2, \ldots)$, where sex ${ }^{(m)}$ is 1 if the $m$ th individual is a male (and 0 otherwise) and where mass ${ }^{(m)}$ denotes the body mass of the $\mathrm{mth}$ individual in grams. In comparison to the HMM for a single animal, we have additionally included the subject-specific covariates 'sex' and 'body mass'. The former divides the population into two groups-females and maleswhile the latter takes account of possible heterogeneity across individuals of different body mass. If that was the only extension of the basic HMM considered above, the model would still have one crucial limitation: it would not allow for different individualities or personalities of the animals. Indeed, it would assume that animals of the same sex and the same body mass act according to exactly the same stochastic principles. As this appears to be unrealistic and too restrictive, we further increase the flexibility of the model by incorporating random effects. To be specific, we assume that the parameters of the statedependent process, $\pi_{1}$ and $\pi_{2}$, are not fixed across subjects, but that each of them is randomly distributed on the interval $[0,1]$, independently and identically across subjects:

$$
\pi_{i, m} \stackrel{i i d}{\sim} B\left(a_{i}, b_{i}\right), \quad m=1, \ldots, M,
$$

for $i=1$ and $i=2$, respectively, where $B\left(a_{i}, b_{i}\right)$ denotes a beta distribution with shape parameters $a_{i}>0$ and $b_{i}>0$, and $\pi_{i, m}$ denotes the probability of feeding, given motivational state $i$, for the $\mathrm{mth}$ individual. Note that we can, in principle, also model correlation between the random effects, e.g. by using a bivariate Gaussian distribution for the vector of the logittransformed parameters $\pi_{1}$ and $\pi_{2}$; for the sake of simplicity and readability we did, however, not attempt this in the current work.

Our model belongs to the flexible class of the socalled mixed HMMs (Altman 2007). The inclusion of random effects offers an elegant and plausible way for modelling 'personality' -in a broad sense-of individuals. On the other hand, the presence of random 
effects unfortunately renders the evaluation and maximization of the likelihood very challenging: in our case with two random effects, the likelihood function involves a twofold integral:

$$
\begin{aligned}
\mathcal{L}= & \prod_{m=1}^{M} \int_{0}^{1} \int_{0}^{1} \delta P_{\pi}\left(x_{1, m}\right) \Gamma^{(1, m)} P_{\pi}\left(x_{2, m}\right) \Gamma^{(2, m)} \cdot \ldots \\
& \ldots \cdot \Gamma^{\left(T_{m}-1, m\right)} P_{\pi}\left(x_{T_{m}, m}\right) 1^{t} f_{1}\left(\pi_{1}\right) f_{2}\left(\pi_{2}\right) \mathrm{d} \pi_{1} d \pi_{2} .
\end{aligned}
$$

Here $x_{t_{m}}$ denotes the observation made at time $t$ for lemur $m$ and $f_{i}$ denotes the probability density function of the $B\left(a_{i}, b_{i}\right)$-distribution. The other ingredients are defined analogously as in equation (2.2); in particular, $\Gamma^{(t, m)}$ is the matrix comprising the state-transition probabilities at time $t$ for the $m$ th individual. For simplicity, the likelihood here is given for the case with only one time series associated with each lemur; indeed, we have more than that. As the different series were recorded on different days, they can reasonably be assumed to be independent, and thus the corresponding extension of the formula given in equation (2.5) is straightforward; see also Altman (2007). Owing to the multiple integral, this likelihood cannot be evaluated directly. We applied numerical integration, i.e. we approximated each of the two integrals by a sum based on partitioning the integration interval into a number of bins and then approximating the integrand within each bin; see the appendix for more details on the type of approximation we applied. Maximization of the likelihood was carried out using $\mathrm{nlm}()$ in R. Numerical integration is computationally expensive, and as the computational burden increases exponentially with the number of random effects, this method can only be applied when there are few random effects. A more sophisticated alternative, which is, however, less accessible to practitioners, is given by Monte Carlo EM methods. For a comprehensive discussion of the existing approaches for estimating HMMs that incorporate random effects, see Altman (2007). A computationally less intensive alternative uses discrete distributions for the random effects (Maruotti and Rydén 2009). 


\section{RESULTS}

The parameter estimates for the mixed HMM, defined by equations (2.3) and (2.4), and associated $95 \%$ confidence intervals $(\mathrm{Cls})^{1}$ are given in table 1 . For the sake of better interpretability, each of the (beta) random effects' distributions, $B\left(a_{i}, b_{i}\right), i=1,2$, has been reparameterized in terms of a mean $\left(\mu_{i}\right)$ and a standard deviation parameter $\left(\sigma_{i}\right)^{2}$.

For each of the covariates - 'time', 'mass' and 'sex' - we conducted a likelihood ratio test of the simplified model (without the respective covariate) against the full model as defined by equations (2.3) and (2.4). At a 5 per cent significance level, the simplified models were rejected in favour of the full model for the covariates 'time' and 'sex', respectively, while the simplified model without covariate 'mass' could not be rejected; the $p$ values are $<0.001,<0.001$ and $<0.127$, for 'time', 'sex' and 'mass', respectively.

TAB. 1: ESTIMATED PARAMETERS WITH 95\% CIS FOR THE MIXED HMM

\begin{tabular}{cccc}
\hline Parameter & Associtaion with & Eestimate & $\mathbf{C l}$ \\
\hline$\delta_{1}$ & & 0.869 & $(0.820,0.906)$ \\
$B_{0,1}$ & & 2.177 & $(1.348,3.005)$ \\
$B_{0,2}$ & time & 1.961 & $(1.055,2.867)$ \\
$B_{1,1}$ & time & 0.0041 & $(0.0031,0.0051)$ \\
$B_{1,2}$ & sex & -0.402 & $(-0.0007,0.0013)$ \\
$B_{2,1}$ & sex & -0.317 & $(-0.603,-0.202)$ \\
$B_{2,2}$ & body mass & 0.0139 & $(-0.0023,0.093)$ \\
$B_{3,1}$ & body mass & -0.0065 & $(-0.0240,0.0111)$ \\
$B_{3,2}$ & random effect $\pi_{1}$ & 0.015 & $(0.013,0.017)$ \\
$\mu_{1}$ & random effect $\pi_{1}$ & 0.004 & $(0.001,0.020)$ \\
$\sigma_{1}$ & random effect $\pi_{2}$ & 0.925 & $(0.892,0.949)$ \\
$\mu_{2}$ & random effect $\pi_{2}$ & 0.058 & $(0.030,0.112)$ \\
$\sigma_{2}$ & & & \\
\hline
\end{tabular}

${ }^{1}$ The $\mathrm{Cls}$ are based on the Hessian of the log-likelihood for the estimated parameters (Zucchini \& MacDonald 2009). Using $\mathrm{nlm}()$ in $\mathrm{R}$, the likelihood was maximized with respect to unconstrained transformed parameters (e.g. the constrained parameter $\mu_{i} \in[0,1]$ was mapped to the real line using a logit link); this method thus gives Cls for the transformed parameters. Approximate Cls for the parameters themselves were obtained by applying the corresponding inverse transformations to the interval boundaries obtained for the transformed parameters.

${ }^{2}$ For given mean $\mu_{i}$ and standard deviation $\sigma_{i}$ the shape parameters of the beta distribution are obtained as $a_{i}=\sigma_{i}^{-2} \mu_{i}^{-2}\left(1-\mu_{i}\right)-\mu_{i}$ and $a_{i}=\mu_{i} \sigma_{i}^{-2}\left(1-\mu_{i}\right)^{2}-\left(1-\mu_{i}\right)$. 
To gain some insight into the goodness-of-fit of the model, we conducted the following simple predictive check: first, for each of the 54 different covariate combinations (corresponding to the 54 different mouse lemurs observed), we simulated series from the fitted model with exactly the same lengths and placements in time as the corresponding observed ones. Figure 2 displays histograms of the subject-specific ratios 'number of feeding events/number of events in total' (i.e. the proportion of observations that correspond to 'feeding'), for the observed data and for one typical set of simulated series (repetitions did not indicate any significant mismatch). This check suggests that the model captures the observed variability in these proportions reasonably adequately, but note that this covers only one arbitrarily chosen aspect of the data-the meaningfulness thus is limited.
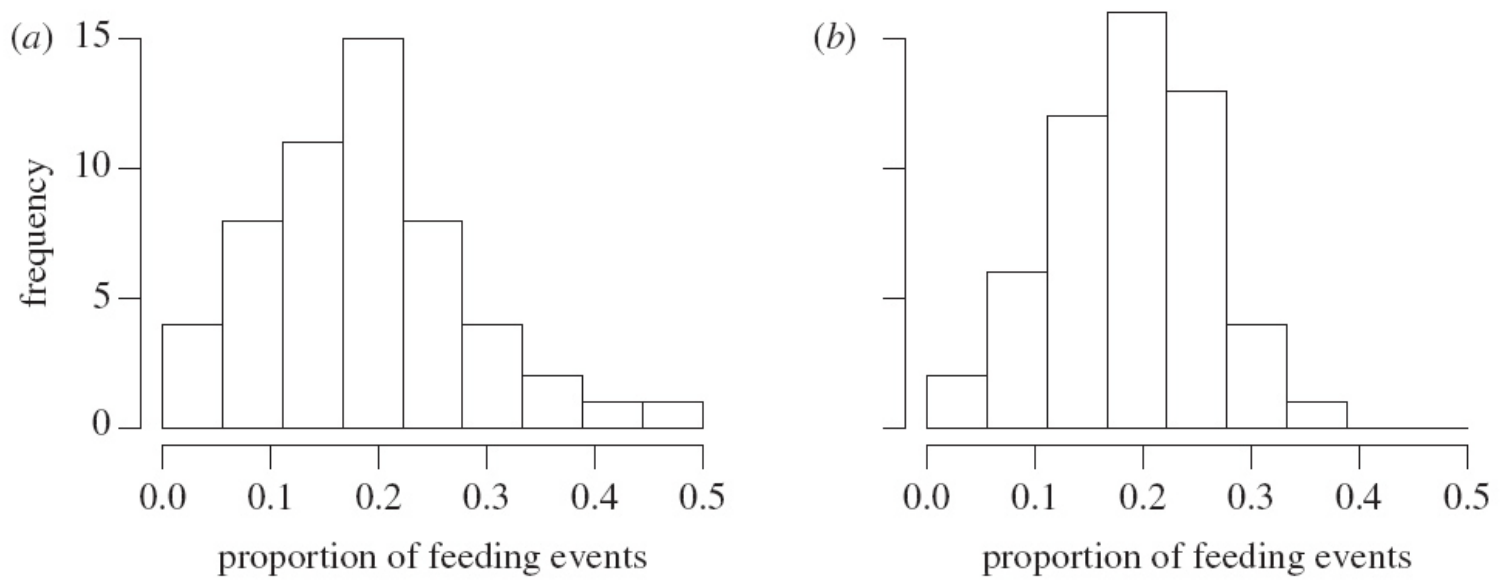

FIG. 2: PREDICTIVE CHECK: HISTOGRAMS OF THE RATIOS 'NUMBER OF FEEDING EVENTS/NUMBER OF EVENTS IN TOTAL', FOR THE (a) OBSERVED AND (b) SIMULATED DATA

The various aspects concerning the (motivational) state process are illustrated in figure 3, which displays the transition probabilities in dependence of the covariates 'mass', 'sex' and 'time of night'. In the following, we list predictions for the feeding behaviour made by the fitted model. Considering the influence of the time of night, mouse lemurs are more likely to switch between the 'satiated' and the 'hungry' motivational state at the beginning of the night than towards the end of the observation period. The behaviour of female grey mouse lemurs is more persistent as reflected by both (stochastically) longer feeding and non-feeding periods, whereas male mouse lemurs change their activity more frequently. Furthermore, mouse lemurs with a high bodymass stay (stochastically) longer satiated and exhibit shorter hungry periods (i.e. they feed less often than light ones). Notably, a female 
with the lowest body mass of $33 \mathrm{~g}$ would still have a more persistent feeding behaviour at a given observation time than a male with the highest body mass of $59 \mathrm{~g}$.
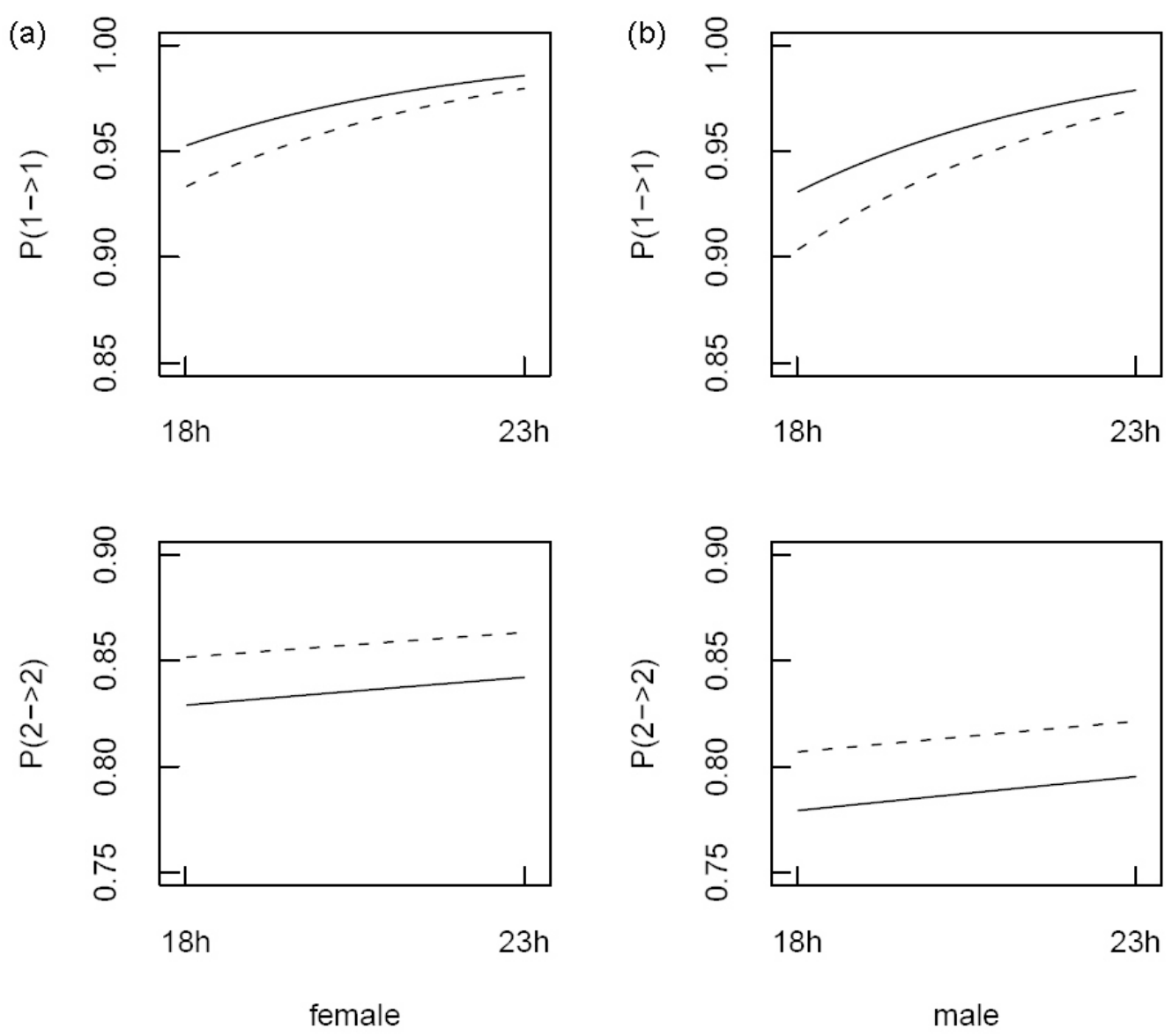

FIG. 3: PROBABILITIES OF MOTIVATIONAL STATE CONSISTENCY IN DEPENDENCE OF THE COVARIATES SEX' ((a) FEMALES, (b) MALES), 'MASS' (SOLID LINES: MASS=59G, DASHED LINES: MASS=33G) AND 'TIME OF NIGHT' (X-AXES); P(I $\rightarrow$ j) REFERS TO $P\left(S_{t+1}=\right.$ $j(S t=i)$

We now consider the state-dependent distributions. The joint distribution of the random effects, $\pi_{1}$ and $\pi_{2}$, is displayed in figure 4 . Notice the scales: the distribution of $\pi_{2}-$ the feeding behaviour in the 'hungry' motivational state-is wider, meaning that the differences across individuals are larger for that parameter. For most animals, $\pi_{2}$ is around the mean, 0.925 , but the density of $\pi_{2}$ has significant mass $\left.\approx 0.27\right)$ even below 0.9 . As regards the probability of feeding in the 'satiated' state, there is much less variability across individuals; according to the fitted model for more than 99 per cent of the lemurs that probability is smaller than 0.03 . Therefore, between individuals of the same sex and same body mass, feeding behaviour in the hungry state is much more variable than in the satiated 
state. When compared with a model with no random effects (i.e. constant $\pi_{1}$ and $\pi_{2}$ across subjects), the Akaike information criterion (AIC) selects the model presented here, i.e. the one that includes random effects ( $\triangle \mathrm{AIC}=29.7)$.

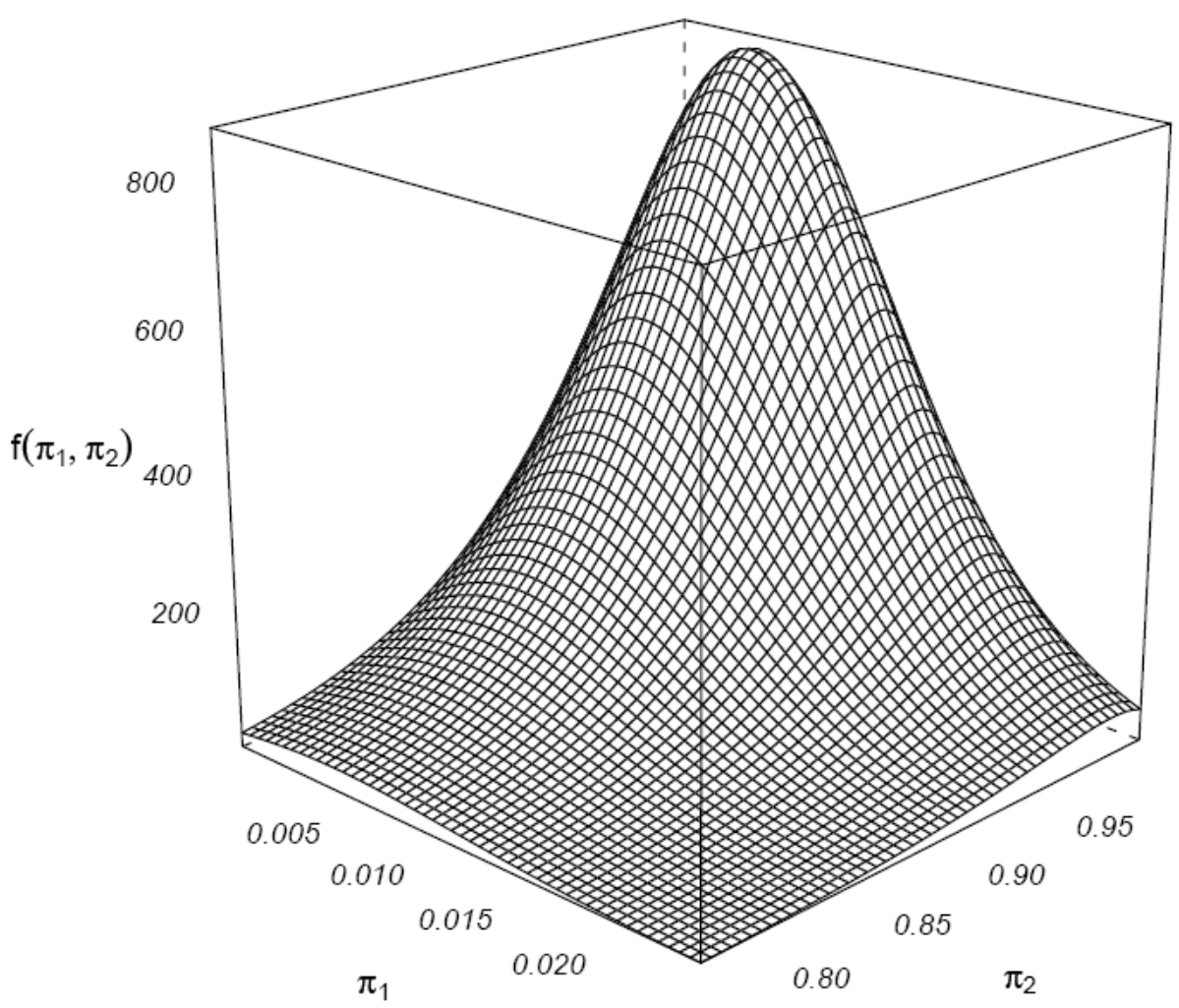

FIG. 4: FITTED JOINT PROBABILITY DENSITY FUNCTION OF $\pi 1(=\mathrm{x})$ AND $\pi 2$ (=y)

\section{DISCUSSION}

Building integrative models is an important step when studying the relationship between proximate behavioural processes and the environment in free-ranging animals (Patterson et al. 2008). We developed a statistical model with high relevance for the study of behavioural processes and underlying motivational aspects.

\subsection{Evaluation of the modelling approach}

HMMs have proved to be very useful for dealing with unmeasured state processes; cf. Zucchini \& MacDonald (2009) for numerous examples. They are immensely flexible and can be applied to different kinds of behaviours, giving them great potential in statistical ecology. They provide increased interpretive capabilities by allowing us to identify transitions in underlying hidden states, even if these transitions are not obvious from observations (Tucker 
and Anand 2005). A (recently increased) number of publications on animal movement took advantage of the flexibility of HMMs to analyse the processes related to individual movement (Franke et al. 2006; Patterson et al. 2009; Pedersen et al. 2011). However, ecological applications of HMMs are still relatively rare and have focused mostly on modelling the behaviour of single individuals separately; an exception is Zucchini et al. (2008).

Including subject-specific covariates in the model enables factors that drive differences in behavioural dynamics across individuals to be identified. HMMs, and in particular such that incorporate covariates, can facilitate detecting differences in behaviour which are not directly obvious from observations; e.g. the same absolute time devoted to actual behaviours might be reached by quite different motivational state sequences. Possible future directions for extending our model are numerous, but perhaps most fruitful will be the inclusion of covariates with more explanatory power, such as measures of physical condition (body mass does not reflect physical condition per se) or a combination of spatial and behavioural data.

Another important aspect of our model is the inclusion of random effects (individuality). Including individuality seemed useful to us for two different reasons. First, the included covariates 'sex' and 'body mass' are not likely to explain all behavioural differences between individuals. Second, individual reactions make the model more realistic, since it is unlikely that individuals react in the same manner. Animal behaviour is usually characterized by a combination of a certain degree of flexibility in behavioural responses on the one hand and consistent differences in behaviours between individuals, the socalled animal personalities, on the other hand. The awareness of this paradox is highlighted by the growing interest in animal personalities (Réale et al. 2005). By using mixed HMMs, behavioural ecologists might be able to identify how behavioural flexibility and personality differences interact and lead to differences in behavioural sequences.

Important possible extensions of the model we considered here include the relaxation of the first-order assumption concerning the state process (which often will be unrealistic). Technically, it is not difficult to fit HMMs with higher order dependencies in the state process (see Zucchini and MacDonald (2009), §8.3), or to consider more flexible distributions for the state dwell times (i.e. the times spent in the motivational states, which 
under the first-order assumption are geometrically distributed, see (Langrock and Zucchini 2011).

\subsection{Influence of covariates on feeding behaviour of grey mouse lemurs}

The results of our model offer new views and hypotheses for future analyses of mouse lemur behaviour. According to the present model, state-switching probability changed with advancing time of night. At the beginning of the night, individuals changed more often between the states associated with either hunger or satiation. It makes intuitive sense to assume that individuals should be hungry at the beginning of their activity period. But why do grey mouse lemurs switch states more often? We know that about 85 per cent of the diet was composed of tree exudates during the observation period (see APPENDIX B). Gum has been defined as a slowly depleting, monopolizable resource (Génin 2003; Dammhahn and Kappeler 2010). Gum trees seem to be most profitable at the beginning of the night because gum production can accumulate bigger drops during the day, whereas gum is regularly harvested during the night. The yield per visited tree is therefore probably higher at the beginning of the night. Possibly, mouse lemurs adjust their behaviour not only to their physiological needs, but also to the availability of the resource. In other words, mouse lemurs switched more often between the 'satiated' and 'hungry' state because they switched their whereabouts more often to patrol the gum trees in their home range.

Regarding the covariate body mass, the model indicated that heavy individuals fed for shorter periods and had longer non-feeding bouts than lighter individuals (but note that this effect was not found to be significant). Body condition has been found to play an important part in the life of grey mouse lemurs. It influences, for example, mating success of males (Eberle and Kappeler 2004b), but also activity patterns of individuals on other temporal scales (Schmid 1999). Potentially, heavier individuals monopolized recourses of higher quality, or they have a generally reduced activity because of higher energetic reserves or as an antipredator strategy.

The sex effect on the consistency of feeding patterns might be related to dominance structures. Studies from captivity suggest that female grey mouse lemurs are dominant over males (Radespiel and Zimmermann 2001). If females are truly dominant over males, they might be much less often displaced from feeding sites than males or monopolize trees of higher productivity. Another possibility for these differences in feeding duration between 
sexes could be the fact that, following natal dispersal, most males are not living in their natal habitat anymore. Unfamiliarity with their new habitat might force males (temporarily) to feed on whatever resource regardless of the quality. Including data on movements, social interactions or number of feeding trees and food availability for a given individual could be useful to untangle the reasons for the observed sex effect. The application of the model to data on adult individuals or data from subadults in different seasons might also be worthwhile.

\section{CONCLUSION}

Based on the evaluation and application of our model, we highlighted the usefulness and advantages of HMMs, in general, and mixed HMMs in particular, for statistical analyses of (multiple) behavioural sequences and the generation of further testable hypotheses, in this case about the feeding behaviour of mouse lemurs and their determinants. Mixed HMMs can help us to derive general organizational mechanisms of behavioural processes and to understand how they influence the ecological dynamics of populations and thus whole ecological environments.

\section{ACKNOWLEDGEMENTS}

The authors would like to thank Walter Zucchini, Ruth King and three anonymous reviewers for helpful comments on earlier versions of this manuscript, and the Ministry of the Environment and Forests of Madagascar and the CNFEREF for their authorization of this study. Field work was conducted with support from the German Excellence Initiative (Courant Centre Evolution of Social Behavior) and the German Primate Center (DPZ). The research of the third author was partly funded by the Engineering and Physical Sciences Research Council (ESPRC reference EP/F069766/1).

All research reported in this manuscript is in compliance with animal care regulations and applicable national laws of Germany and Madagascar. All research protocols were approved by the appropriate Animal Use and Care committees of Germany (Bundesministerium für Naturschutz, BfN) and Madagascar (Ministe`re de l'Environnement et des Eaux et Forêts, MINEEF). 


\section{APPENDIX A. DETAILS ON THE NUMERICAL INTEGRATION}

The details concerning the numerical integration that has been applied to evaluate the likelihood equation (2.5) are as follows. After initial experiments that aimed at identifying the 'essential range' of the two random effects' distributions (i.e. the range where almost all mass of the distributions is in) each of these ranges, for the two random effects' distributions as indexed by $r, r=1 / 4 \quad 1,2$, was split into $q$ equally sized intervals $W_{i, r}=\left(w_{1-i, r}, w_{i, r}\right), \mathrm{i}=1, \ldots, \mathrm{q}$. Let $w_{i, r}^{*}$ denote the midpoint of $\mathrm{W}_{\mathrm{i}, r}$, and let $\mathrm{h}(\pi, m)$ denote the likelihood for given $\pi=\left(\pi_{1}, \pi_{2}\right)$ and individual $m$ :

$$
\begin{aligned}
h(\pi, m)= & \delta P_{\pi}\left(x_{1, m}\right) \Gamma^{(1, m)} P_{\pi}\left(x_{2, m}\right) \Gamma^{(2, m)} \cdot \ldots . \\
& \cdot \Gamma^{\left(T_{m}-1, m\right)} P_{\pi}\left(x_{T, m}\right) 1^{t} .
\end{aligned}
$$

The likelihood equation (2.5) can then be approximated as follows:

equation (2.5)

$$
\begin{gathered}
=\prod_{m=1}^{M} \int_{0}^{1} \int_{0}^{1} h(\pi, m) f_{1}\left(\pi_{1}\right) f_{2}\left(\pi_{2}\right) d \pi_{1} d \pi_{2} \\
\approx \prod_{m=1}^{M} \sum_{i=1}^{q} \prod_{j=1}^{q} \int_{w_{i-1,1}}^{w_{i, 1}} \times \int_{w_{j-1,2}}^{w_{j, 1}} h(\pi, m) f_{1}\left(\pi_{1}\right) f_{2}\left(\pi_{2}\right) d \pi_{1} d \pi_{2} \\
\approx \prod_{m=1}^{M} \sum_{i=1}^{q} \prod_{j=1}^{q} \int_{w_{i-1,1}}^{w_{i, 1}} \times \int_{w_{j-1,2}}^{w_{j, 1}} h\left(\left(w_{i, 1}^{*}, w_{j, 2}^{*}\right), m\right) f_{1}\left(\pi_{1}\right) f_{2}\left(\pi_{2}\right) d \pi_{1} d \pi_{2} \\
\approx \prod_{m=1}^{M} \sum_{i=1}^{q} \prod_{j=1}^{q} \int_{w_{i-1,1}}^{w_{i, 1}} \times \int_{w_{j-1,2}}^{w_{j, 1}} h\left(\left(w_{i, 1}^{*}, w_{j, 2}^{*}\right), m\right)\left(F_{1}\left(w_{i, 1}\right)-\left(F_{1}\left(w_{i-1,1}\right) F_{2}\left(w_{j, 2}\right)\right.\right. \\
\left.-F_{2}\left(w_{j-1,2}\right)\right),
\end{gathered}
$$

where $F_{i}$ denotes the cumulative distribution function associated with the density $f_{i}, i=1,2$. There are two sources of approximation: first, the replacement of the intervals $[0,1]$ by the respective essential ranges (second line above), and second, the replacement of the function $\mathrm{h}(\pi, m)$ by the constant value of that function evaluated at the midpoints of the respective intervals (fourth line above). The former is not necessary in the present application (since we are dealing with the bounded interval $[0,1])$, however, as long as the essential range is chosen to be sufficiently large, it improves the approximation since the intervals $W_{i, r}$ become narrower and the discretization thus finer (and note that this step is necessary in applications where the integration intervals are unbounded). Note that this is by no means 
the only way in which the integrals can be approximated: one may, for example, apply more sophisticated methods such as Gauss-Legendre quadrature.

\section{APPENDIX B. TIME SPEND FEEDING AND DIET COMPOSITION}

TAB. B1: PROPORTION OF OBSERVATION TIME, DURING WHICH ANIMALS WERE OBSERVED TO FEED

\begin{tabular}{ccc}
\hline Sex & Non-feeding $(\%)$ & Feeding $(\%)$ \\
\hline Females & 85.6 & 14.4 \\
Males & 84.8 & 15.2 \\
\hline
\end{tabular}

TAB. B2: DIET COMNPOSITION DETERMINED AS TIME SEEN FEEDING ON DIFFERENT FOOD RESOURCES IN (\%)

\begin{tabular}{ccccc}
\hline Sex & Gum & Animal matter * & Unspecified ** & Insect secretions \\
\hline Females & 84.0 & 11.5 & 3.3 & 1.2 \\
Males & 86.2 & 10.4 & 3.4 & 0 \\
\hline
\end{tabular}

* Animal matter: Invertebrates and small vertebrates

** Unspecified: Unspecified food sources. The animal was seen feeding, but the food source could not be identified 
The ghost of inbreeding avoidance Consequences of natal dispersal for inbreeding risk in a small solitary mammal, the grey mouse lemur

with Cornelia Kraus, Elise Huchard, Manfred Eberle and Peter M. Kappeler 


\section{ABSTRACT}

An increasing number of studies in different biological fields have addressed the potential functional link between sex-biased dispersal and the consequences of inbreeding depression such as reduced survival. A central, albeit unresolved question in this context is whether natal dispersal can resolve inbreeding risk completely. We assessed the consequences of sex-biased natal dispersal for the presence of inbreeding depression and inbreeding risk in a population of grey mouse lemurs (Microcebus murinus), small solitary primates from Western Madagascar. We statistically modelled the effect of heterozygosity on individual survival using a 10-generation dataset collected during a long-term capture-mark-recapture study and heterozygosity estimates derived from microsatellite data. We found no support for a negative effect of low levels of heterozygosity on survival probabilities and relative low values did not prevent individuals from reaching high ages. We also assessed the frequency of inbreeding risk between close relatives and the presence of mate bias towards genetically more dissimilar mates based on parentage analyses. First-order relatives were simultaneously present during mating seasons at relatively high frequencies (15-17\%), but they never reproduced with each other. High mortality, high number of available mates and promiscuity further reduced the risk of inbreeding. A bias of parentage towards less related mates, as a further mechanism to enhance outbreeding could not be detected. Thus, in grey mouse lemurs sex-biased natal dispersal is associated with a low inbreeding risk, but the causal contingencies between these two factors remain unresolved.

Keywords: sex-biased dispersal, inbreeding risk, inbreeding avoidance, mate bias, Microcebus murinus

\section{INTRODUCTION}

Breeding between close relatives occurs only at low frequencies in most wild populations. This outcome is most likely the result of strong selection against inbreeding depression. Inbreeding depression is defined as a decline of fitness as a result of mating between related individuals relative to the fitness of progeny between unrelated individuals (Charlesworth and Charlesworth 1987; Charlesworth and Charlesworth 1999; Ives and Whitlock 2002; Roff 2002; Charlesworth and Willis 2009). Reduced fitness of inbred individuals is the result of 
deleterious allelic combinations and/or a reduced reaction scope of the immune system due to genome-wide increased homozygosity. Fitness-related traits found to be affected by inbreeding are birth weight (Coltman et al. 1998; Coulson et al. 1998), development (Diehl and Koehn 1985; Charpentier et al. 2006; Nielsen et al. 2012), reproductive success (Foerster et al. 2006; Zeh and Zeh 2006), resistance to disease and environmental stress (Coltman et al. 1999; Acevedo-Whitehouse et al. 2003), and survival during different life stages, especially during early life stages (Stockley et al. 1993; Markert et al. 2004; Cohas et al. 2009). Using polymorphic genetic markers to estimate individual heterozygosity, the association of general genome-wide genetic diversity with fitness components has been studied in a variety of species (reviewed in Kempenaers 2007). Fitness is supposed to increase linearly with increasing heterozygosity, though some studies found that intermediate values present the optimal degree of heterozygosity (quadratic relationship), when a species experiences some degree of outbreeding depression (Aparicio et al. 2001).

However, the biological significance of positive associations between individual genetic diversity (heterozygosity) and fitness-related traits reported in these studies remains debated (Balloux et al. 2004; Aparicio et al. 2006; Kempenaers 2007). Main criticisms are a publication bias in favour of positive results and the unevaluated biological relevance of low degrees of correlation between fitness-related traits and estimated levels of heterozygosity. Still, individuals could benefit from biasing parentage in favour of genetically dissimilar mates that will maximise genetic diversity of offspring, even if it does not per se serve to avoid inbreeding (Amos et al. 2001). Beside age-related effects of heterozygosity with younger individuals suffering higher levels of inbreeding depression (an example of statedependent effects, Cohas et al. 2009), stressful environmental conditions seem to increase the magnitude of heterozygosity-fitness correlations (Kempenaers 2007; Cohas et al. 2009). Hence, increased genetic diversity of offspring could serve as a preparation for a temporally fluctuating environment (genetic bet-hedging) which can be important for dispersing individuals (Brown 1997; Fox and Rauter 2003; Kempenaers 2007; Marshall et al. 2008; Oh and Badyaev 2008; Fromhage et al. 2009; Selonen and Hanski 2010).

In turn, dispersal has long been regarded as an important mechanism for inbreeding avoidance. Inbreeding avoidance is also one of the most frequently mentioned ultimate causes for the existence of sex-biased dispersal (SBD) rates (Greenwood 1980; Dobson 1982; Waser et al. 1986; Perrin and Goudet 2001; Guillaume and Perrin 2006; Clutton-Brock and 
McAuliffe 2009; Lukas and Clutton-Brock 2011; Clutton-Brock and Lukas 2012). In theoretical scenarios, evolutionary stable strategies of SBD evolve when inbreeding avoidance is combined with other factors like the degree of asymmetry between sexes in local competition for resources and mating opportunities, the degree of genetic load (the amount of inbreeding depression), kin selection, dispersal cost and dispersal tendencies of the opposite sex (Perrin and Mazalov 2000). Inbreeding avoidance is assumed to further accentuate existing contrasts because higher philopatry of one sex increases inbreeding risk and thereby constitutes an additional pressure to trigger the evolution of SBD (Perrin and Mazalov 1999; Perrin and Mazalov 2000; Perrin and Goudet 2001; Lehmann and Perrin 2003).

However, the function of SBD as an inbreeding avoidance strategy has been and remains debated, because dispersal does not always effectively eliminate inbreeding risk (Moore and Ali 1984; 1985; Waser et al. 1986; Pusey 1987; Pusey 1988; Perrin and Mazalov 2000; Perrin and Goudet 2001; Lehmann and Perrin 2003; Lukas and Clutton-Brock 2011; Clutton-Brock and Lukas 2012). Some studies found that breeding with close relatives occurs frequently despite SBD (Foerster et al. 2006; Olson et al. 2012), sometimes even with no apparent consequences (e.g. Duarte et al. 2003). Also, recent comparative analyses of group-living mammals highlighted the fact that individuals of the habitually philopatric sex can find themselves quite regularly in a situation where their potential mating partners are close relatives (Lukas and Clutton-Brock 2011; Clutton-Brock and Lukas 2012). Therefore, habitual dispersal of one sex does not necessarily eliminate the risk of consanguineous matings, if subsequently tenure length of the dispersing sex exceeds the time until sexual maturation of the philopatric sex. In such cases, further mechanisms of inbreeding avoidance might be selected for. These mechanisms include reproductive suppression (Blouin and Blouin 1988; Wolff 1992; O'Riain et al. 2000), delayed reproduction (Cooney and Bennett 2000), matings outside the usual social unit (Burland et al. 2001; Foerster et al. 2003), and kin discrimination (Krackow and Matuschak 1991; Constable et al. 2001; Muniz et al. 2006; Charpentier et al. 2007). Intergenerational overlap in reproductive activity might also be an important explanatory variable for differences in the extent of the sex-bias and for the evolution of female-biased dispersal in polygynous mammal species.

The problem of overlapping reproductive activity between parents and offspring of the opposite sexes can theoretically be extended to non-gregarious species, especially when 
dispersal activity is limited to natal dispersal of one sex. Because these non-gregarious species typically lack parental care, inbreeding avoidance cannot be mediated through familiarity and alternative mechanisms might become necessary. One such mechanism is phenotype matching, where relatives can be identified through one or more phenotypic traits, and can subsequently be avoided as mates (Widdig 2007). This mechanism is likely to be selected in a species in which fathers and offspring have no contact.

The present study investigates the consequences of natal dispersal for the risk of inbreeding and the presence of inbreeding depression in a solitary primate, the grey mouse lemur (Microcebus murinus). These small (60g body mass), nocturnal primates are distributed along Southern and Western Madagascar (Kappeler and Rasoloarison 2003). Grey mouse lemurs represent the typical mammalian pattern of SBD with a strong male dispersal bias with regard to propensities and distances (Radespiel et al. 2001; Eberle and Kappeler 2002; Kappeler and Rasoloarison 2003; Fredsted et al. 2005; Gligor et al. 2009). Beside consistency between study areas, this pattern appears to be very stable among years (Wimmer et al. 2002; Fredsted et al. 2004). The observed strong bias and the absence of regular secondary dispersal might indicate that inbreeding risk is low. However, the fast life history of mouse lemurs creates a potential for inbreeding. Individuals can reproduce for the first time at an age of 9 months and then every year thereafter. Mortality rates of grey mouse lemurs - especially in the first year of life - are high (Kraus et al. 2008) resulting in high population turnover rates. Still, some individuals survive up to 6-10 years in the wild. Hence, females start reproducing while their father could still be reproductively active. Another potential source of inbreeding between male and female relatives can result from occasional philopatry of males or dispersal of males over small distances. Mate bias towards less related individuals could be used as a strategy to ensure inbreeding avoidance in this situation.

Using microsatellite markers, previous studies found that grey mouse lemurs did not bias matings towards less related partners, but that females biased paternity in favour of males with more dissimilar MHC-constitutions (Schwensow et al. 2008). These results allow several interpretations. First, inbreeding avoidance strategies might be non-effective despite a significant inbreeding risk, and the study population might suffer from inbreeding depression. Testing this possibility requires investigating the consequences of inbreeding depression on fitness-related traits like survival. Second, inbreeding avoidance strategies 
might exist, but go undetected in this analytical design. While based on a reasonable sample size (74 offspring with known parents), Schwensow et al. (2008) compared chosen to random mates without accounting for spatial structure. Because distance between males and females does influence the probability of mating success (Eberle and Kappeler 2004b), the set of candidate mates for each female should more realistically include only neighbouring males. Testing this possibility requires testing the existence of inbreeding avoidance while controlling for the spatial structure of the population. Third, inbreeding risk might be low (also due to natal dispersal), leading to a low, fitness-irrelevant level of genome-wide heterozygosity and thereby limiting the intensity of selection for additional inbreeding avoidance strategies. Testing this possibility requires evaluating the extent of inbreeding risk. Here we target these open questions and extend previous analyses by quantifying the extent of (1) inbreeding depression, (2) inbreeding avoidance and (3) inbreeding risk (indexed by inbreeding effects in survival) by combining a 10-generations dataset of a capture-mark-recapture (CMR) study with genetic data.

\section{METHODS}

\subsection{Study population and trapping procedures}

The study population is located within a 12,500 ha forestry concession of the Centre National de Formation, d'Etude et de Recherche en Environnement et Foresterie (CNFFREF) in Kirindy Forest (Kappeler and Fichtel 2012a). Since 1994, DNA samples and population parameters have been collected during monthly CMR sessions using about 160 traps at a time in an area of about 9ha within a 60-ha grid system, locally known as CS7. Additional captures in surrounding areas were conducted once or twice a year and covered an area of about 18ha. For trapping, Sherman live traps were baited with small pieces of banana and positioned near trail intersections at dusk on three consecutive nights. Captured animals were collected at dawn, and handled according to the protocol of Eberle and Kappeler (2002). Standard procedures included individual marking with subdermally injected transponders (or reidentifying recaptured individuals). 


\subsection{Microsatellite DNA analyses}

DNA was isolated from ear biopsies, using the QIAGEN QIAamp Tissue Kit for DNA Purification (Quiagen) (Eberle and Kappeler 2004b). Twelve different polymorphic microsatellite systems with an average number of 22 alleles (range: 13-39) were used for analyses (Mm06, MmF3, Pvc 9.2, Pvc a.1: Wimmer et al. 2002; 33104, Mm22, Mm39, Mm40, Mm42, Mm43b, Mm51, Mm60: Hapke et al. 2003). The presence of null alleles, stutter errors or short allele dominance was tested with the program MICRO-CHECKER v. 2.2.3 (Van Oosterhout et al. 2004). Loci were tested for selective neutrality based on the $\mathrm{F}_{\text {ST }}$ outlier method (fdist) (Beaumont and Nichols 1996) implemented in the software LOSITAN (Antao et al. 2008) with a total of 95,000 simulations and the options 'neutral mean $\mathrm{F}_{\mathrm{ST}}$ ' and 'force mean $\mathrm{F}_{\mathrm{ST}}{ }^{\prime}$. This analysis requires data of two populations. Since we had only data from one population, we used genetic data from the years 2000 and 2010 for this analysis of selective neutrality. All individuals present in 2000 had been replaced by 2010, which avoided pseudoreplication. This analysis indicated selective neutrality for all loci.

\subsection{Calculation of relatedness estimates and heterozygosity}

Parentage analyses for determination of true parents and distances between arithmetic trapping position were based on combined mismatch and likelihood analysis using CERVUS 3 (Kalinowski et al. 2007) and COLONY v. 2.0.1.9 (Jones and Wang 2010). A maximum of two homozygous mismatches or one heterozygous mismatch was accepted, to sort candidate parents. The likelihood analysis for non-excluded candidates in CERVUS 3 was based on detailed parentage simulations to estimate the resolving power of all loci and to estimate critical values to evaluate the parentage analysis statistically $(100,000$ runs, 94 candidate parents, assumptions: sampling rate $=0.95$; average loci typing rate $=0.85$; error rate $=0.05$; one close relative of the true parent among the other candidate parents).

For relatedness estimation we used the triadic IBD relatedness estimates (Wang 2007) calculated with the software COANCESTRY v. 1.0.0.0 (Wang 2011) for all individuals captured between the years 1999 and 2010, using 100 reference individuals and 100 bootstraps. To estimate individual genome-wide heterozygosity, we calculated the homozygosity by loci (HL) index of Aparicio et al. (2006). We restrict the results presented here to the $\mathrm{HL}$ index because it has been shown to be a better correlate of genome-wide homozygosity and inbreeding coefficients than two other estimators of heterozygosity, 
internal relatedness (IR) or uncorrected homozygosity (HO) (Aparicio, 2006). For our study population HL estimates were highly correlated with IR ( $r=0.98$, Amos et al. 2001) and another estimator of heterozygosity, the standardized heterozygosity estimator $(\mathrm{SH}, r=-0.98$, Coltman et al. 1999). Analyses based on these estimators resulted in analogous conclusions.

\subsection{Heterozygosity and survival}

To determine whether heterozygosity affected survival probabilities, we used a two-step approach (similar to Cohas et al. 2009). We first modelled survival and recapture probabilities using capture-mark recapture models (e.g. Lebreton et al. 1992). We selected the most parsimonious model out of a candidate set of models using Akaike's Information Criterion AIC (e.g. Burnham and Anderson 2002). In the second step we added heterozygosity as an individual covariate to this basic model to test specific hypotheses regarding survival consequences of variation in heterozygosity using likelihood-ratio tests (LRT).

\subsubsection{Capture-mark-recapture data}

To model survival probabilities, we used CMR data from 1999 to 2011. We did not include data from before 1999 because too few animals from these cohorts were genotyped. As described in Kraus et al. (2008), we estimated seasonal survival using data from the main trapping session at the onset of the austral winter (April/May) and the one at the end of the dry season (onset of summer), before the mating season starts in October. We could not use the summer trapping session from 2004, since it was conducted too late. Hence, we created a dummy trapping session ("10 October") and fixed its recapture probabilities at 0 . The complete data set included 481 animals (294 males, 187 females) for which we have heterozygosity estimates and which were caught a total of 1031 times.

\subsubsection{CMR model}

We used the Cormack-Jolly-Seber model for open populations (CJS, Cormack 1964; Jolly 1965; Seber 1965) implemented in the program MARK (White and Burnham 1999) to statistically model survival $(\phi)$ and recapture probabilities $(p)$. Model selection was based on Akaike's Information Criterion AIC or one of its appropriate variants (here $\mathrm{QAIC}_{c}$ which adjusts for small sample sizes and the presence of overdispersion, Burnham and Anderson 
2002). We interpreted model selection results in a weight of evidence context based on QAICC differences $\left(\Delta_{i}\right)$ and normalised Akaike weights $\left(w_{i}\right)$ as described by Burnham and Anderson (2002). Briefly, $\Delta_{i}$ is the difference between the AIC of the top model vs. the model considered, and thus reflects the likelihood of a given model relative to the best-supported model which has the lowest AIC. Akaike weights derive from this measure and are normalised (i.e. weights of all models in the set sum to 1 ).

We first assessed the goodness-of-fit of global models using the median- $\hat{c}$ approach implemented in program MARK. The variance inflation factor $\hat{c}$ was estimated to be slightly above 1 ( $\hat{c}=1.09)$, indicating a low level of extra-binomial variance. We still adjusted model selection statistics (QAIC $c$, QDeviance) accordingly. For the basic seasonal survival model we considered the factors factor sex $(s)$, age $(a)$ and time $(t)$ in our models. To evaluate statedetermined effects of heterozygosity and account for high mortality in the first year of live (Kraus et al. 2008), age was represented by three age classes, juveniles (juv, 3-9 months old, i.e., 1st winter), yearlings (yrl: 10-16 months old, i.e. first summer, first breeding season) and adults (ad: >16 months old). Our candidate model set was partly based on a priori knowledge from an earlier study on seasonal survival of the same mouse lemur population which comprised the years 1995 to 2005 (Kraus et al. 2008). As our global model (GM) we again used $\phi^{\mathrm{W}}\left(a^{*} s+t\right) \phi^{\mathrm{S}}\left(a^{*} s+t\right) p^{\mathrm{W}}\left(a^{*} s+t\right) p^{\mathrm{S}}\left(a^{*} s+t\right)$ (W: winter, S: summer, *: interactive effect, +: additive effect). A fully interactive global model would have been overparameterised, because recapture probabilities vary strongly among trapping sessions due to the highly variable weather conditions and resource availability, which decreases the ratio between effective samples size and the number of parameters.

This analysis uses CMR data from 1999-2011, and hence only partially overlaps with the data set from the earlier study. Moreover, strong population fluctuations were observed between 2005 and 2011. Therefore we did not simply use the top model from that analysis for further inference, but included candidate models incorporating model terms that received some support in the confidence set of models established in that analysis (all models with a relative likelihood $>0.05$, Kraus et al. 2008). Model selection in the first analysis strongly supported temporal variation in summer survival as well as recapture probabilities in winter and summer. In contrast we found no evidence for temporal variation in winter survival. All candidate models for winter survival included an age effect, because natal male dispersal in the Kirindy population takes place between April and September 
(Eberle and Kappeler 2004). With the CJS-model we cannot separate emigration and mortality, and hence estimates for juvenile males represent so-called "apparent survival" probabilities. We do know that female dispersal and/or secondary male dispersal are at most very rare events in this population (Eberle and Kappeler 2004b) and thus, we feel confident that estimates for these sex-age-classes represent "true survival" probabilities. Hence, our candidate models for winter and summer recapture probabilities, as well as for summer survival included $a^{*} s+t(\mathrm{GM}), a+s+t, a+t, s+t$ and $t$. For winter survival we used $a^{*} s+t, a^{*} s, \operatorname{juv}(s) \operatorname{ad}($.$) and a$.

In order to limit the total number of models, we selected the most parsimonious model for each major model part (i.e. survival winter, survival summer, recapture summer, recapture winter) against the global model for the remaining model parts. We then built our basic survival and recapture model by combining the selected models for each part.

\subsubsection{Effects of heterozygosity}

To test for an association between heterozygosity levels and survival, we added our heterozygosity estimate $(\mathrm{HL})$ as an individual covariate to the most parsimonious model for survival and recapture probabilities (the basic model). Because the basic model and those incorporating heterozygosity effects are nested, we compared these models using likelihood ratio tests (LRTs, $a=0.05$ ). Our LRTs aimed to address 3 specific hypotheses. (1) We tested for an overall effect of heterozygosity on mouse lemur survival. We excluded juvenile males from the heterozygosity effect, because for these we cannot distinguish between survival and emigration (see above). (2) Based on the idea that heterozygosity effects can be statespecific (here state=age, Cohas et al. 2009), we added the heterozygosity effect only for juveniles females (i.e. first winter survival). (3) To evaluate the hypothesis that heterozygosity effects are exacerbated under more harsh conditions (Kempenaers 2007), we added the heterozygosity effect only to summer survival, and, respectively, only to male summer survival, because survival was found to be substantially lower in summer and even more so in males (see also Kraus et al. 2008). Because heterozygosity estimates were rather high we always tested for a linear and for a quadratic effect. Linear effects were expected to show improved survival chances with increasing heterozygosity. Quadratic effects would represent an optimal heterozygosity level between inbreeding and outbreeding depression. 
None of the effects tested were statistically significant; therefore we did not correct $p$ values for multiple testing.

\subsection{Assignment of candidate mates for females and males}

For females, males were considered candidate mates, if they fulfilled the following two criteria: (1) the average distance between partners is lower than the maximum distance recorded between the two parents of an offspring using parentage data (Schliehe-Diecks, unpublished data) from our population ( $n=125$ parent pairs, mean distance among parents $\pm S D=99 \pm 62$, range: $0-319 \mathrm{~m}),(2)$ individual's presence in the last capture preceding

the mating season in September or subsequent capture sessions. This second criterion is conservative and tends to under-estimate the number of mates available, since some males might be present but not captured on such occasion, but it ensures that only live males were included in the analysis.

A total of 51 females were included into the data set, based on (1) their ranging pattern within the core of the study area and (2) on the status as being part of a parentoffspring triad between 2000 and 2010. For these females, we could evaluate a possible mate bias based on offspring from one to eight seasons, resulting in a total of 81 mating sessions with 103 chosen fathers. 329 different males were included into the candidate male set, with numbers of candidate mates per mating session per female ranging between 24 and 71 males (mean $\pm S D=52 \pm 12$ ).

Criteria to include females as candidate mates for a given male were similar as described above. In total, we had data on $n=118$ (118 mating sessions from $n_{\text {males }}=83$ covering 1-7 mating seasons and 162 chosen mates, no. of candidate mates=18-79, mean $\pm S D=52 \pm 13, n_{\text {females }}=319$ ). Except for one case (distance $=453 \mathrm{~m}$ ), males sired offspring within a radius of $333 \mathrm{~m}$, and consequently we included only females living within this distance as a conservative criterion (for qualifying an individual as potential partner: $n=170$ parent pairs, mean distance among parents $\pm S D=126 \pm 79 \mathrm{~m}$ ).

\subsection{Inbreeding risk}

Data on relatives of first order (parent-offspring and full sibling dyads) simultaneously present during mating seasons was, again, inferred from parentage analyses and pedigree data (Schliehe-Diecks, unpublished data). Using this information, we scanned through our 
data sets of candidate mates to mark mating sessions, which included relatives of first order. Inbreeding risk was quantified as the frequency at which this situation occurred in relation to the total number of mating sessions. Mating sessions with inbreeding risk were separately reanalysed to test whether individuals reacted to the potential risk of inbreeding by biasing parentage towards less related individuals.

\subsection{Permutation test of mate choice and inbreeding avoidance}

First, we tested whether females bias paternity of their offspring according to the genotypes of candidate mates. The distribution for the relatedness of random partners to the female, and their heterozygosity indices (HL) was generated by randomly matching 10,000 times each female to one male of her pool of candidate mates. A distribution of these parameters under the null hypothesis was generated by randomly allocating males to groups 10,000 times. In each case, the $p$ value was computed as the proportion of cases displaying a lower or equal mean of pairwise relatedness value, or HL-index than the observed one.

Randomization tests allowed for multiple appearances of individuals in the dataset. This is inevitable in a system where both home ranges and generations are overlapping (meaning that a male will be candidate for several females - and vice-versa - and that each individual male and female may be present for several consecutive seasons). Although two different pairs cannot be considered as statistically independent if they involve the same male or female, using permutation tests limits the risk of false statistical inference in such a design. For instance, an individual possessing a rare genotype may increase the risk of falsepositives if it makes multiple appearances, but our permutation test controls for this bias because such an individual will appear as many times in the random as in the observed distribution.

We then reran these simulation tests excluding males that were in their first mating season $(n=274)$, as previous work has shown that they are significantly less likely than older males to sire offspring (Eberle and Kappeler 2004b). This resulted in a data set of 56 mating sessions from 40 females with $13-42$ candidate mates (mean $\pm S D=31 \pm 9$ ). Finally, we investigated whether females reacted to the presence of first order relatives by repeating the analysis for mating nights during which a first order relative was present.

We repeated the analysis described above for data sets of males to test whether males might choose females that are less related or have higher heterozygosity indices than 
random mates. We did not rerun the analyses after excluding young females as females typically reproduce in their first mating season (Eberle and Kappeler 2004a; Huchard et al. 2011).

\section{RESULTS}

\subsection{Heterozygosity and survival}

The most parsimonious survival model selected from our candidate set was the same as in the earlier study (covering the years 1995-2005), and parameter estimates were similar, suggesting that the survival patterns found are quite representative for this population (Tab. 1, Kraus et al. 2008). There was little model selection uncertainty in choosing the most parsimonious model for recapture probabilities and summer survival: an additive effect of sex to temporal variation was strongly supported for each of these model parts $\left(p^{\mathrm{W}}\right.$ : $\left.w_{+}(s+t)=0.93, p^{s}: w_{+}(s+t)=0.87, \phi^{s}: w_{+}(s+t)=0.75\right)$. Summer survival probabilities varied between 0.38 and 0.84 among years with female survival being slightly above male survival (geometric means $\phi_{\text {females }}^{\mathrm{S}}=0.61 \pm 0.09 \mathrm{SE}, \quad 95 \% \mathrm{Cls}=[0.40 ; 0.77], \quad \phi_{\text {males }}^{\mathrm{S}}=0.55 \pm 0.10 \mathrm{SE}$, $95 \% \mathrm{Cls}=[0.35 ; 0.73]$, all probabilities are given on a semi-annual time-scale). In contrast, winter survival was rather constant over the years $\left(w_{+}(\right.$no $\left.t)=0.99\right)$, and higher than summer survival, with juveniles surviving less well than adults $\left(\phi^{\mathrm{W}}{ }_{\text {ad males }}=\phi^{\mathrm{W}}{ }_{\text {ad females }}=0.88 \pm 0.04 \mathrm{SE}\right.$, $95 \% \mathrm{Cls}=[0.77 ; 0.94], \phi^{\mathrm{W}}$ juv females $=0.75 \pm 0.08 \mathrm{SE}, 95 \% \mathrm{Cls}=[0.56 ; 0.87], \phi^{\mathrm{W}}$ juv males $=0.52 \pm 0.06 \mathrm{SE}$, $95 \% \mathrm{Cls}=[0.41 ; 0.62])$, all probabilities are given on a semi-annual time-scale). We cannot currently estimate how much of the difference between juvenile male and female survival is due to male natal dispersal. We did not find any statistical evidence for a linear or quadratic effect of heterozygosity on either overall (excluding juvenile males), juvenile female, summer, or male summer survival (Tab. 2).

\subsection{Inbreeding risk}

For females, we identified 15 mating sessions (17\% of all analysed mating nights) for 14 individuals during which either a son $(n=7)$ or a father $(n=8)$ of a female were present as potential mates. Number of candidate mates for these mating sessions ranged between 24 and 71 individuals (mean $\pm S D=53 \pm 8$ ), which is well above the previously described numbers of approaching (range: 2-15males) and actually mated males (range: 1-7 males) (Eberle and Kappeler, 2004b). 
TAB. 1: MODEL SELECTION STATISTICS FOR RECAPTURE PROBABILITIES IN (a) WINTER (pW) AND (b) SUMMER (pW), AND FOR APPARENT SURVIVAL PROBABILITIES IN WINTER $\left(\phi^{\mathrm{W}}\right)$ AND SUMMER $\left(\phi^{\mathrm{S}}\right)$ NESTED IN THE MOST GENERAL MODEL FOR THE REMAINING COMPONENTS $\left(\mathrm{A}^{*} \mathrm{~S}+\mathrm{T}\right)$

Factors considered are age (a; juv: juveniles, ad: adults), sex (s) and year ( $\mathrm{t}$ ). Model notation: (.) constant, * interaction, + additive effect (parallel lines on a logit-scale). The number of estimable parameters (K), The Quasi-likelihood adjusted deviance (QDEV), Akaike's Information Criterion (QAICC), the difference between the minimum QAICC of the top model and the model considered $(\triangle \mathrm{i})$ and Akaike weights (wi) are given for each model

\begin{tabular}{|c|c|c|c|c|c|c|}
\hline Rank & Model $i$ & $K$ & QDEV & $\mathrm{QAIC}_{c}$ & $\Delta_{i}$ & $w_{i}$ \\
\hline \multicolumn{7}{|c|}{ (a) Recapture probabilities in winter $p^{\mathrm{W}}$ depend on: } \\
\hline 1 & $s+t$ & 58 & 1743.22 & 1866.45 & 0 & 0.65 \\
\hline 2 & $a+s+t$ & 59 & 1743.21 & 1868.70 & 2.25 & 0.21 \\
\hline 3 & $a^{*} s+t$ & 60 & 1743.19 & 1870.94 & 4.49 & 0.07 \\
\hline 4 & $a+t$ & 58 & 1748.51 & 1871.75 & 5.30 & 0.05 \\
\hline 5 & $t$ & 57 & 1752.36 & 1873.34 & 6.89 & 0.02 \\
\hline \multicolumn{7}{|c|}{ (b) Recapture probabilities in summer $p^{S}$ depend on: } \\
\hline 1 & $s+t$ & 58 & 1745.10 & 1868.33 & 0 & 0.43 \\
\hline 2 & $a+s+t$ & 59 & 1743.36 & 1868.86 & 0.53 & 0.33 \\
\hline 3 & $a^{*} s+t$ & 60 & 1743.19 & 1870.94 & 2.61 & 0.12 \\
\hline 4 & $t$ & 57 & 1751.01 & 1872.00 & 3.67 & 0.07 \\
\hline 5 & $a+t$ & 58 & 1749.43 & 1872.66 & 4.33 & 0.05 \\
\hline \multicolumn{7}{|c|}{ (c) Survival probabilities in winter $\phi^{\mathrm{W}}$ depend on: } \\
\hline 1 & juv(s)ad(.) & 48 & 1762.84 & 1863.76 & 0 & 0.39 \\
\hline 2 & $a+s$ & 48 & 1763.20 & 1864.12 & 0.36 & 0.33 \\
\hline 3 & $a$ & 47 & 1767.17 & 1865.89 & 2.13 & 0.14 \\
\hline 4 & $a^{*} s$ & 49 & 1762.77 & 1865.92 & 2.16 & 0.13 \\
\hline 5 & $a^{*} s+t$ & 60 & 1743.19 & 1870.94 & 7.18 & 0.01 \\
\hline \multicolumn{7}{|c|}{ (d) Survival probabilities in summer $\phi^{\text {s }}$ depend on } \\
\hline 1 & $s+t$ & 58 & 1743.45 & 1866.68 & 0 & 0.50 \\
\hline 2 & $t$ & 57 & 1747.67 & 1868.65 & 1.97 & 0.19 \\
\hline 3 & $a+s+t$ & 59 & 1743.19 & 1868.68 & 2.00 & 0.19 \\
\hline 4 & $a+t$ & 58 & 1747.60 & 1870.84 & 4.16 & 0.06 \\
\hline 5 & $a^{*} s+t$ & 60 & 1743.19 & 1870.94 & 4.26 & 0.06 \\
\hline
\end{tabular}


TAB. 2: LIKELIHOOD RATIO TESTS (ADJUSTED FOR $\hat{c}=1.09$ ) OF HETEROZYGOSITY (HL12) EFFECTS ON SURVIVAL. MODELS INCORPORATING THE HETEROZYGOSITY EFFECT (GENERAL MODELS) WERE TESTED AGAINST THE REDUCED BASIC MODEL $\left(\phi^{\mathrm{W}}\left(\mathrm{juv}(s) \operatorname{ad}(.) \phi^{\mathrm{s}}(s+t) p^{\mathrm{W}}(s+t) p^{\mathrm{s}}(s+t)\right)\right.$

Shown are the effects tested, and the survival model term that were added in the general survival model as well as the test statistic $\left(\chi^{2}\right)$, degrees of freedom (d.f.) and the $p$ value of the LRT

\begin{tabular}{|c|c|c|c|c|}
\hline Hypothesis tested & general survival model term & $x^{2}$ & d.f. & $\boldsymbol{P}$ \\
\hline $\begin{array}{l}\text { linear overall* effect of } \\
\mathrm{HL} \text { on survival }\end{array}$ & $\phi^{a l l}(\mathrm{HL})$ & 1.05 & 1 & 0.30 \\
\hline $\begin{array}{l}\text { quadratic overall* effect } \\
\text { of } \mathrm{HL} \text { on survival }\end{array}$ & $\phi^{a l l}\left(H L+H L^{2}\right)$ & 1.62 & 2 & 0.45 \\
\hline $\begin{array}{l}\text { linear effect of } \mathrm{HL} \text { on } \\
\text { juvenile female survival }\end{array}$ & $\phi^{W,} j^{\mathrm{uvF}}(\mathrm{HL})$ & 0.51 & 1 & 0.48 \\
\hline $\begin{array}{l}\text { quadratic effect of } \mathrm{HL} \text { on } \\
\text { juvenile female survival }\end{array}$ & $\phi^{W,} j^{\mathrm{uvF}}\left(\mathrm{HL}+\mathrm{HL}^{2}\right)$ & 2.06 & 2 & 0.36 \\
\hline $\begin{array}{l}\text { linear effect of } \mathrm{HL} \text { on } \\
\text { summer survival }\end{array}$ & $\phi^{\mathrm{S}}(\mathrm{HL})$ & 1.26 & 1 & 0.26 \\
\hline $\begin{array}{l}\text { quadratic effect of } \mathrm{HL} \text { on } \\
\text { summer survival }\end{array}$ & $\phi^{S}\left(H L+H L^{2}\right)$ & 1.90 & 2 & 0.39 \\
\hline $\begin{array}{l}\text { linear effect of } \mathrm{HL} \text { on male } \\
\text { summer survival }\end{array}$ & $\phi^{\mathrm{S}, \mathrm{M}}(\mathrm{HL})$ & 1.17 & 1 & 0.28 \\
\hline $\begin{array}{l}\text { quadratic effect of } \mathrm{HL} \text { on } \\
\text { male summer survival }\end{array}$ & $\phi^{S, M}\left(H L+H L^{2}\right)$ & 1.17 & 2 & 0.56 \\
\hline
\end{tabular}

Nineteen mating sessions (15\%) of 15 males were identified during which first order relatives were present. In 5 of the 19 mating sessions two first degree relatives were present (four times two daughters and once the mother and a daughter of a male). In the remaining 14 mating sessions, a mother $(n=6)$, a daughter $(n=7)$ or a full-sister of the male was present. 


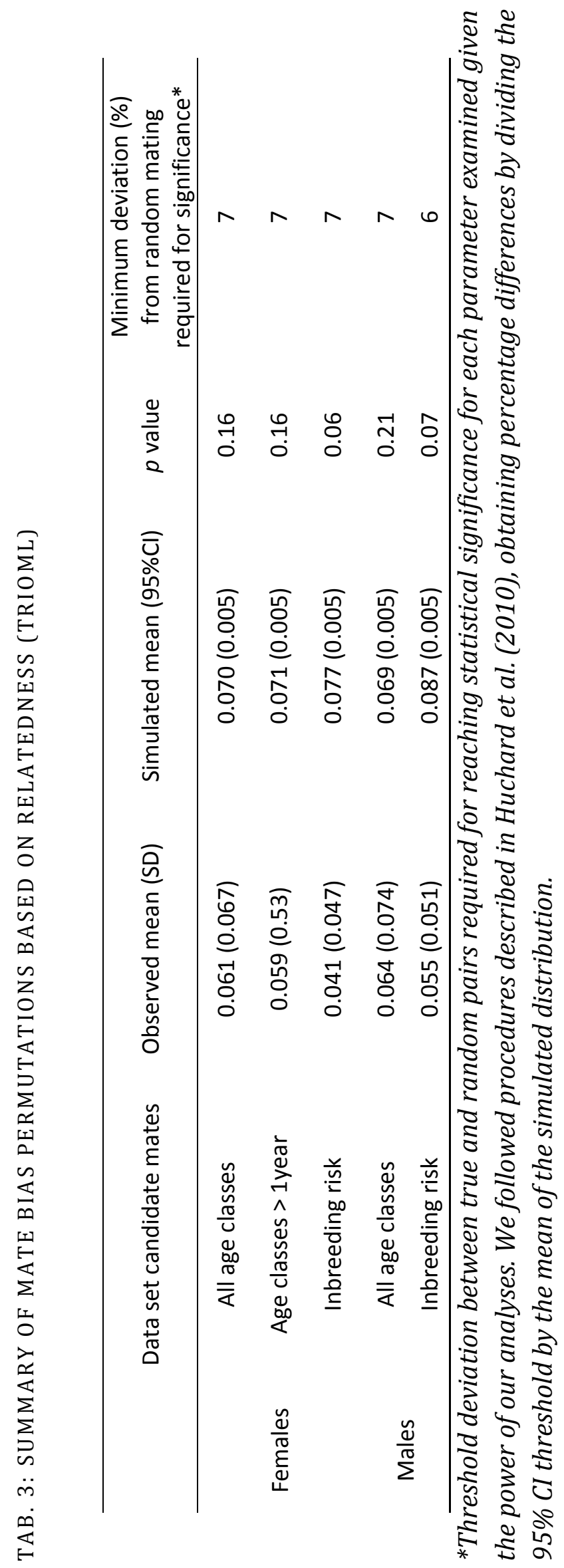




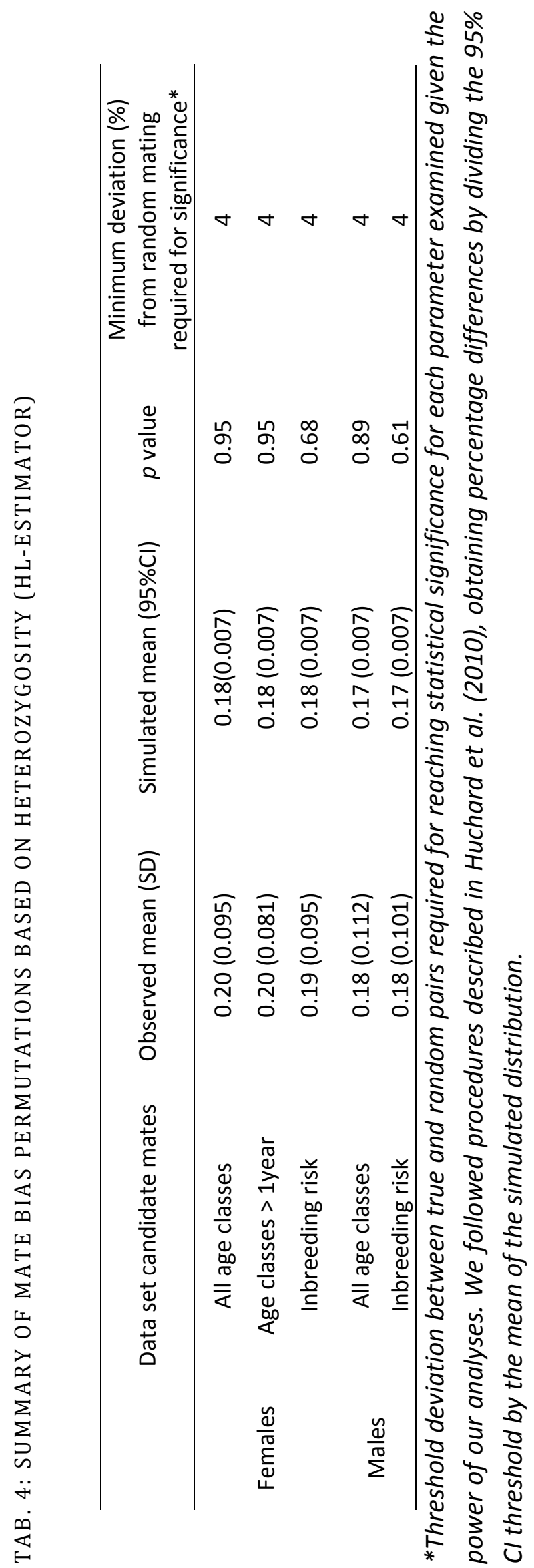



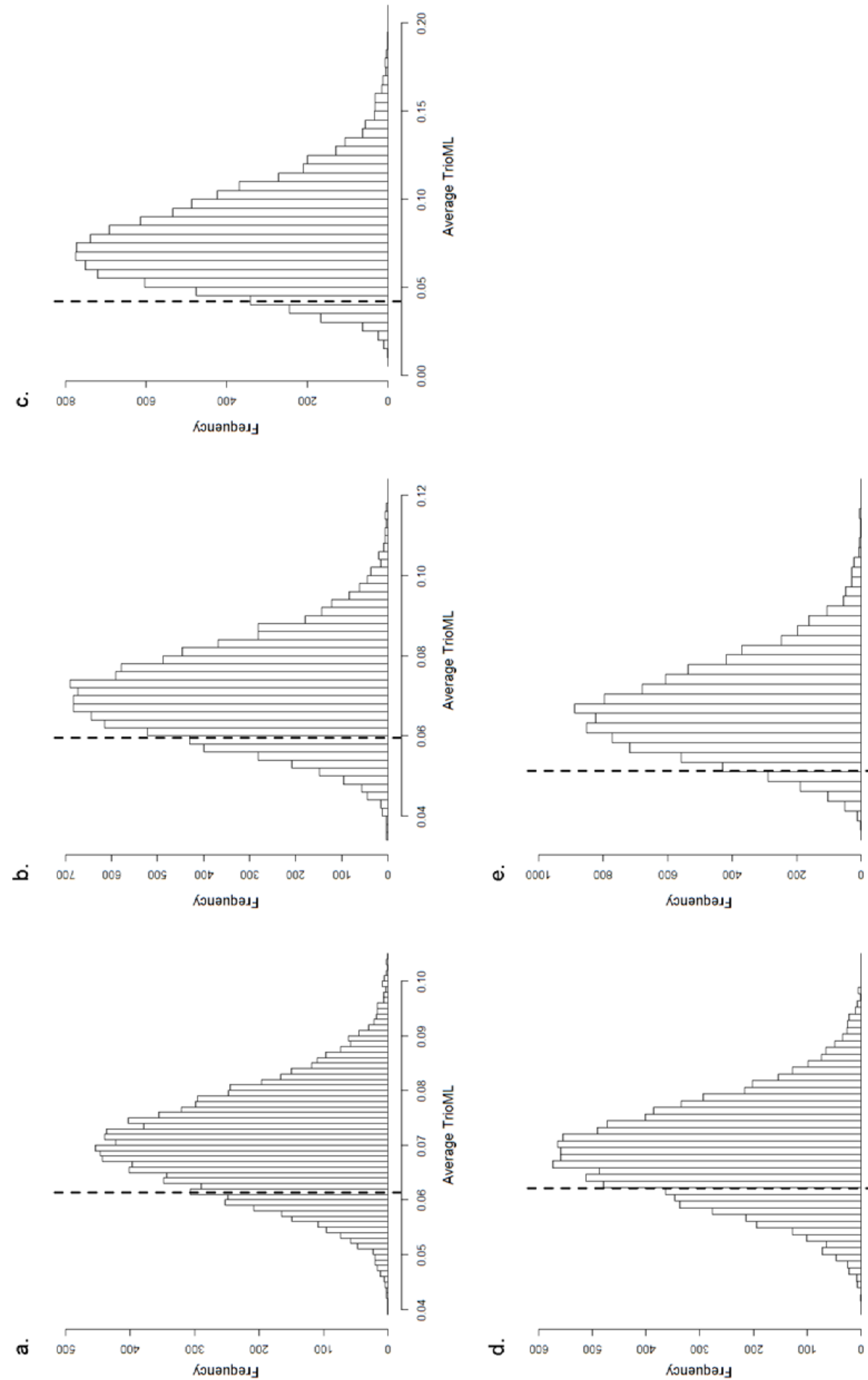

H 


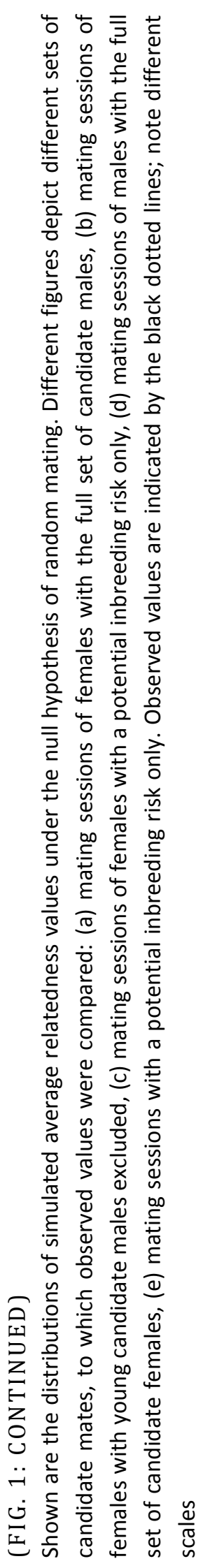


CHAPTER 4: CONSEQUENCES OF NATAL DISPERSAL
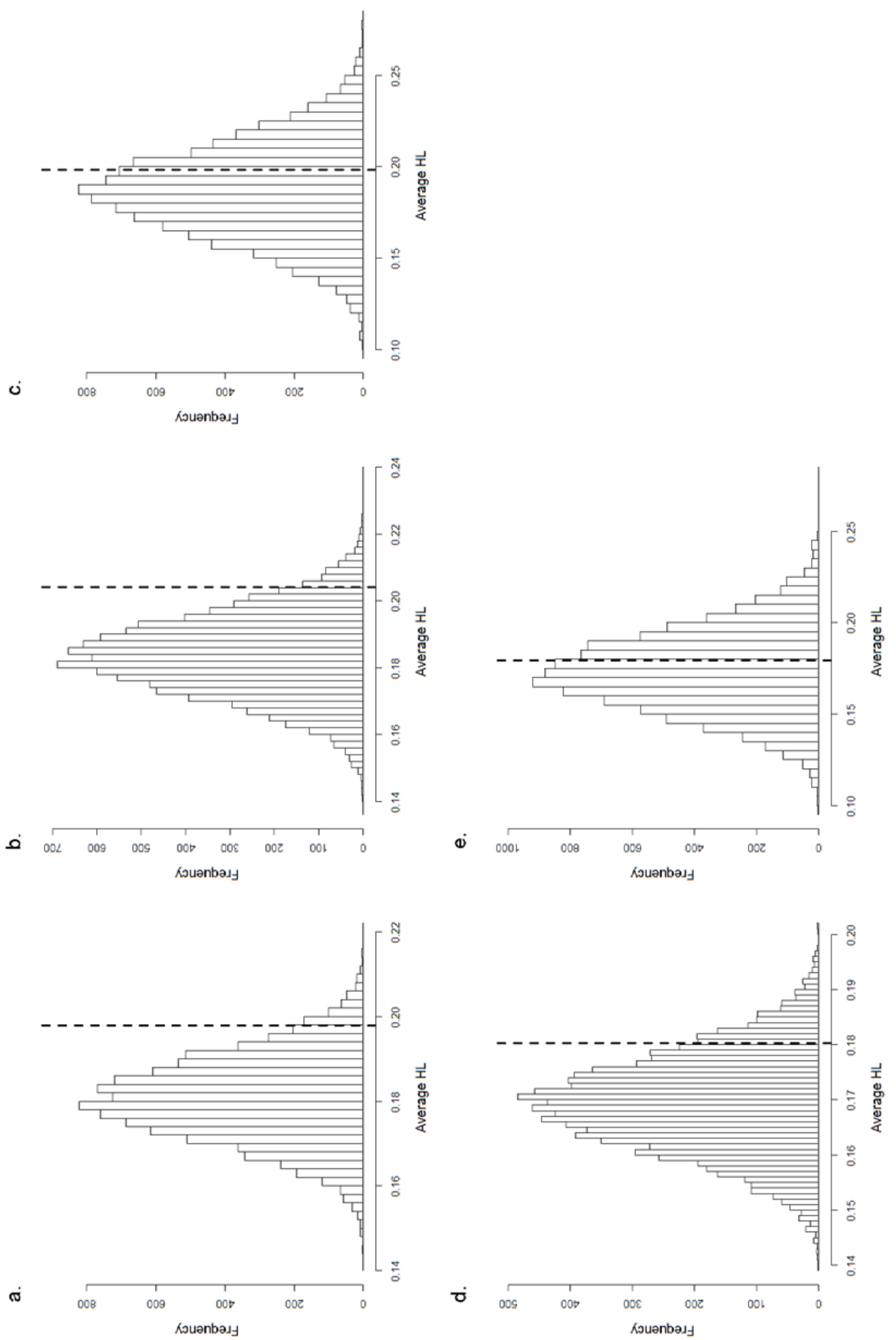

品

章

$\circ$

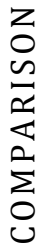




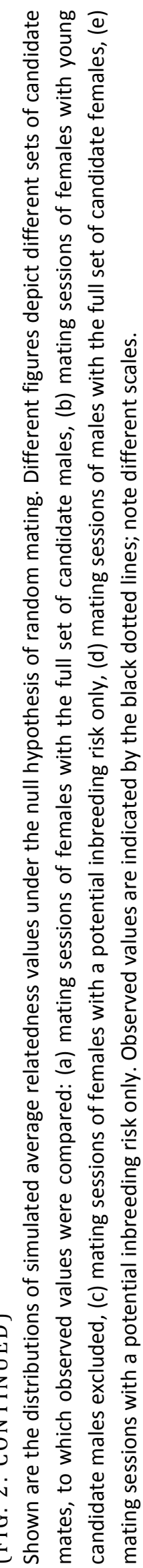




\subsection{Inbreeding avoidance}

Neither females nor males seemed to bias maternity or paternity according to the investigated genetic measures. Average relatedness of chosen mates did not differ from simulated relatedness between random dyads, though there was a tendency towards lower relatedness of chosen mates for females and males (Tab. 3, Fig. 1). The same pattern was evident when we excluded young males from the candidate list of mates. Eighteen to 71 candidate mates were present during these mating sessions (mean $\pm S D=50 \pm 13$ ). Hence, based on the presence of relatives during mating seasons a moderate risk of inbreeding for both males and females does exist. Individual genetic diversity of mates could also not be distinguished from random mating patterns (Tab. 4, Fig. 2). However, contrary to our expectations, chosen mates tended to be more homozygous than random mates (Tab. 4).

For mating sessions, during which the potential of breeding with a relative of firstorder existed ("inbreeding risk", Tab. 3, 4, Fig. 1, 2) average relatedness and levels of individual genetic diversity of chosen mates did again not differ from randomly associated dyads. However, no cases of breeding between relatives of first order could be detected. In pedigrees covering 10 years the closest proven case of reproduction between individuals with a common ancestor in this population was a coupling of aunt and nephew (inbreeding coefficient $(f) \approx 0.125)$. For true parents with relatively high dyadic TrioML $(>0.20)$, we were not able to detect any close family relationships.

\section{DISCUSSION}

We investigated the influence of a singular dispersal event restricted to one sex on the extent of (1) fitness costs of breeding with relatives and (2) inbreeding avoidance. Based on a 10-generation data set, this study evaluated effects of genome-wide heterozygosity on the survival probability of grey mouse lemurs. We did not find a relationship between survival and individual genetic diversity. Further, we assessed the degree of inbreeding risk following natal dispersal and whether mates were discriminated based on relatedness as an additional mechanism to increase outbreeding. The combination of natal dispersal with promiscuity, roaming of males and high mortality made inbreeding between co-occurring close relatives an extremely rare event. Low average relatedness of random mates in this population made it impossible to distinguish whether individuals bias matings in favour of less related mates. 
However, this could also indicate that differences in relatedness and neutral genetic diversity might be of minor importance in this population.

\subsection{Inbreeding depression: heterozygosity and survival}

A common effect of reduced individual genetic diversity is a decreased survival, especially during early life stages (Stockley et al. 1993; Coltman et al. 1998; Coulson et al. 1998; Acevedo-Whitehouse et al. 2003; Markert et al. 2004; Cohas et al. 2009; Ridley et al. 2011). As a consequence of different mortality of subadults, variation in heterozygosity should decrease in older age classes (Diehl and Koehn 1985; Cohas et al. 2009). For our population of grey mouse lemurs patterns of seasonal survival, conformed to results of a previous study (Kraus et al. 2008). Survival of adult individuals was similar for males and females during winter (dry season) and higher than survival of juveniles, but adult male survival was worse during summer (rainy/mating season). For subadults, survival estimates of males during winter were additionally lower than those of females, but during this life stage disappearances can also be caused by dispersal of males. However, we were not able to detect a positive effect of heterozygosity on survival probability, irrespective of whether we considered state-dependant (age) or environmental influences (season) which are supposed to alter the magnitude of genome-wide heterozygosity effects on fitness (Balloux et al. 2004; Brouwer et al. 2007; Kempenaers 2007; Cohas et al. 2009). We also did not find any support for an optimal HL level balancing potential costs of inbreeding and outbreeding depression. Therefore, our findings add support to the notion that effects of genome-wide heterozygosity are rather low to undetectable in large, natural populations.

It has been argued that a number of markers below 10 could be too low to capture genuine genome-wide effects of inbreeding (Balloux et al. 2004; Aparicio et al. 2006). There has indeed been a long debate regarding the use of such indices to measure inbreeding, which is ideally inferred from a pedigree (Coltman and Slate 2003; Balloux et al. 2004). However, the HL estimator is supposed to require only a relatively small number of loci (1015) and to have a better ability to detect heterozygosity fitness correlations than other measures of heterozygosity assuming the relationship between genome-wide heterozygosity and fitness traits coefficients in open populations (Aparicio et al. 2006). Therefore, our results support the contention that effects of genome wide heterozygosity on survival are rather low in large populations (Balloux et al. 2004; Overall et al. 2005). In this context, our 
findings contribute important information regarding the relative importance of the effect of heterozygosity on survival in undisturbed populations, and can also provide a baseline for comparisons with small or fragmented populations.

\subsection{Inbreeding risk}

If dispersal evolved as an effective mechanism to resolve inbreeding risk, the potential of inbreeding should be low in grey mouse lemurs with strong sex-bias in natal dispersal. However, mating sessions with a risk of breeding with a first-order relative occurred for both sexes at a frequency that seemed not negligible (females: $17 \%$, males: $15 \%$ ) and these numbers did not include relatives of second or lower order. Therefore, natal dispersal was not sufficient by itself to eliminate the risk of inbreeding completely. We still never detected a case of close inbreeding in pedigrees and the co-occurrence of first degree relatives of different sexes, of which at least one individual reproduced successfully, was usually limited to one mating season (maximum: two years). Low numbers of available mates could be another incentive to tolerate inbreeding (Duarte et al. 2003). However, for grey mouse lemurs numbers of alternative candidate mates were high and well above previously described numbers of males that actually mated with estrous females (Eberle and Kappeler 2004b). Mating with multiple partners might represent another strategy to reduce the costs of inbreeding (Stockley et al. 1993; Tregenza and Wedell 2000; Foerster et al. 2006) and mating forays outside the usual range can further enhance outbreeding by increasing the set of candidate males for a given female (Winters and Waser 2003). Therefore, in grey mouse lemurs the combination of natal dispersal with promiscuity (Eberle and Kappeler 2004a; Eberle et al. 2007), roaming of males outside habitual home ranges during mating season (Eberle and Kappeler 2004b) and high mortality (Kraus et al. 2008) seems to create enough dilution to reduce inbreeding risk to such a low extent that secondary dispersal as an inbreeding avoidance strategy becomes unnecessary. In fact, adult grey mouse lemurs (>1year) are highly faithful to their home ranges in Kirindy Forest (S. Schliehe-Diecks, unpublished data). Relocations of home ranges, comparable to dispersal of subadult males (described in Chapter 1) have never been observed for adults in our study population.

Dispersal of individuals as a reaction to the presence of their opposite-sex parent or siblings has been used as indirect evidence for the importance of inbreeding avoidance for the evolution of SBD (Bollinger et al. 1993). In grey mouse lemurs, female dispersal occurs 
rarely. However, it seems unlikely that this is a proximately triggered response to inbreeding risk for several reasons. First, even if females might have neighbouring relatives, they do not seem to face a shortage of unrelated candidate mates. Second, but more importantly, females usually disperse over shorter distances than males (Radespiel et al. 2003), which would not necessarily remove them from the mating perimeter of relatives. Finally, based on theoretical models, local resource competition plays an important role in the evolution of female dispersal probabilities (Lehmann and Perrin 2003). In grey mouse lemurs, home range overlap between (related) females might become a problem when the family size is high or the amount of available resources is low (Dammhahn and Kappeler 2009). Therefore, female dispersal could be a response to stress caused by high local resource competition rather than to an increased inbreeding risk. Females of diurnal group-living lemurs indeed do show high levels of competition for breeding slots (Kappeler and Fichtel 2012a; b). However, whether natal dispersal of subadult females and males is influenced by the presence or absence of relatives remains to be evaluated.

\subsection{Inbreeding avoidance}

Our results suggest that the absence of inbreeding does not reflect active inbreeding avoidance through mate choice. Average relatedness of chosen mates did not differ from randomly assigned partners neither for females nor for males, though observed values tended to be lower than simulated means. These results were independent of whether we excluded young males from the analysis of female mate bias or whether we included only sessions, during which the potential for breeding with a relative of first order existed. Our results are congruent with previous studies, which found no effect of microsatellite-based relatedness estimates on mate bias (Schwensow et al. 2008). Hence, grey mouse lemurs did not seem to discriminate mates to maximise outbreeding. Average relatedness of random mates was low and mate bias might therefore not be necessary or not detectable in our study population. It remains possible that mouse lemurs choose mates based on other genetic measures, such as for instance MHC dissimilarity, diversity or particular MHC genotypes. Such a strategy might allow them to optimise offspring MHC diversity and increase their immunocompetence. Results of a previous study support this notion (Schwensow et al. 2008), though these results might need to be confirmed, as they did not control for spatial distance among mates. 
Similarly, we could not detect any mate choice for more heterozygous partners. Chosen mates tended to have lower average heterozygosity than simulated means and observed values of heterozygosity of this study were slightly higher than previously described values (Schwensow et al. 2008). Schwensow et al. (2008) also found that chosen mates had microsatellite alleles that had smaller distances to each other than those of candidate mates. This could indicate that intermediate levels of heterozygosity and outbreeding are advantageous (Aparicio et al. 2001; Kempenaers 2007). However, we found no indication in our survival analysis for such an effect. Positive effects of heterozygosity based on neutral markers on mate bias seem to be rather rare, possibly because they do not provide indirect benefits since heterozygosity is not heritable (Kempenaers 2007). In grey mouse lemurs, the lack of relevance could also be the result of multiple matings, which would already increase offspring diversity (Eberle and Kappeler 2004b). Stronger effects of heterozygosity on mate bias might also be restricted to stressful environmental conditions or in fragmented populations, where the average genetic diversity is reduced (Balloux et al. 2004; Cohas et al. 2009) and when intrasexual competition favours heterozygous partners.

\section{CONCLUSIONS}

In grey mouse lemurs, sex-biased natal dispersal was apparently not fully efficient at controlling inbreeding risk. Indeed, the occurrence of opposite-sex relatives living close to each other was not rare, due to a combination of overlapping generations, fast sexual development and apparent absence of secondary dispersal. Cases of inbreeding among firstorder relatives were never recorded over 10 years in our population, and consequently, we could not detect inbreeding depression based on survival rates. The absence of inbreeding was apparently not explained by active avoidance mechanisms based on selective mating. Nevertheless, average relatedness among candidate mates was low, probably reflecting a combined effect of dispersal with other factors such as promiscuity.

Our study indicates that dispersal behaviour of individuals observed in the present might be less often affected and proximately triggered by a potential inbreeding risk than is often assumed. Given the relative inflexibility of the dispersal propensities of grey mouse lemurs, SBD seems to have become an almost fixed event in the life history of male grey mouse lemurs, which is rather insensible to external cues. This invariable pattern probably persists, because following natal dispersal an already substantially reduced inbreeding risk is 
further decreased through demographic (such as mortality) and behavioural factors (such as male roaming and perhaps the fact that females mate with multiple males). Hence, the possible selective pressures on the evolution of SBD caused by inbreeding avoidance in the past might not be measurable anymore in present, undisturbed populations. A comparison between undisturbed and fragmented populations or such of different size or densities could help understand what consequences would arise, once a system is out of balance.

\section{ACKNOWLEDGEMENTS}

We acknowledge the authorization of this study by the Commission Tripartite and the CAFF of the Direction des Eaux et Forêts, the CNFEREF. All research reported here is in compliance with animal care regulations and applicable national laws of Germany and Madagascar. The appropriate Animal Use and Care committees of Germany (Bundesministerium für Naturschutz, BfN) and Madagascar (Ministère de l'Environnement et des Eaux et Forêts, MINEEF) approved all research protocols. The CRCESP provided financial support. We thank Dr. Rodin Rasoloarison, Léonard Razafimanantsoa, Tiana Andrianjanahary, Bruno Tsiveramana, Remy de Ampataka and the Equipe Kirindy for support and assistance in the field. 
CHAPTER 4: CONSEQUENCES OF NATAL DISPERSAL 


\section{GENERAL DISCUSSION}

Dispersal holds a central role in ecology, evolution and conservation, since it influences the distribution of individuals in space and time. Consequently, much effort has been dedicated into studying the processes, which lead to the emergence of specific dispersal patterns such as sex-biased dispersal (SBD). In this thesis, I investigated different proximate aspects of the dispersal process of a small solitary primate with male-biased dispersal, the grey mouse lemurs (Chapter 1 and Chapter 2). Furthermore, I introduced a method which allows to determine general differences in motivational states between individuals using behavioural sequences (Chapter 3), which presents a useful tool not only for studies on dispersal. Finally, in Chapter 4, I evaluated the consequences of sex-biased dispersal from an evolutionary perspective. In this general discussion I will summarize the major findings, set them into perspective of our current understanding of dispersal and compare them to available data to identify general patterns. Finally, I will outline some future directions for the study of dispersal to fill the gaps in our knowledge of the dispersal process and to develop our understanding of the evolution of SBD.

\section{DISPERSAL MOVEMENTS IN GREY MOUSE LEMURS}

This thesis provided one of the most detailed documentation of mammalian dispersal movements available so far. Movement strategies during the transient phase of dispersal were characterized by a high consistency within and between individuals, but individual differences in the duration of the transfer phase and the distances moved (Chapter 1). Highly directed dispersal pathways seem to be a strategy used by different taxa and species with varying social organisation (Baker 1969; Storm et al. 1976; Baars 1979; Holekamp 1986; Bearder 1987; Mech 1987; Rogers 1987; Wiens and Zitzmann 2003). For grey mouse lemurs, it probably represents, on the one hand, a strategy that balances the need for gathering information for settlement decisions with costs of transfer and, on the other hand, allows males to change back and forth easily between old and prospected home range. Since I was able to provoke similar highly directed movements during a translocation experiment (Chapter 1), it seems that individuals pursue this strategy for large scale explorations.

My observations of dispersal behaviour in grey mouse lemurs indicated that the clear separation of the three distinguished dispersal phases of emigration, transfer and 
immigration (see for example Lidicker and Stenseth 1992; Ronce 2007) is not universally valid. For grey mouse lemurs the transfer and immigration phase are intertwined through their commuting behaviour. I suggested that commuting served as a strategy to deal with unfamiliarity with the new environment, which seems to present the most challenging part of the dispersal process in grey mouse lemurs. Therefore, depending on the duration and form of the dispersal phases, species apparently vary in regard to the main source of dispersal-related costs. Consequently, they should also vary in the strategies to reduce these costs. Mechanisms to reduce dispersal costs in other species include parallel dispersal of several individuals or transfer between neighbouring groups to lower predation risk (Harrison 1992; Roper et al. 2003). Transferring back and forth between old and new site or group seems to be more general mechanism (Holekamp 1986; Rood 1987; Smale et al. 1997; Roper et al. 2003). If dispersal strategies evolved to mitigate transfer costs, this implies that we might have to reassess and refine assumptions about the general severity of the transfer phase in terms of survival and energetic costs. In this regard, it would be extremely valuable to know how much diversity or uniformity other species exhibit in dispersal strategies under different environmental conditions, to disentangle the benefits of different movement styles.

Another factor requiring a more thorough consideration is the relationship between dispersal capacities, habitat choice and landscape matrices. In many theoretical considerations habitats are usually divided into suitable and unsuitable areas (Ims and Yoccoz 1997; Zollner and Lima 1999). In some cases, this seems justified because differences between components of the landscape matrix are obvious. For ruffed grouse, for example, transfer increases mortality, because dispersers often move out of forest covered areas and transfer through more open landscapes, where dispersers become more vulnerable to predation (Yoder et al. 2004). In other cases habitat fragmentation causes obvious differences in suitability of habitats, which alters dispersal behaviour and success of individuals (Matthysen and Currie 1996). However, for seemingly continuous habitats it remains difficult to investigate how landscapes are perceived by dispersers, how this affects movements and how landscape matrices and species-specific dispersal capacities are related to each other, as long as we have no information about dispersal capacities and behaviour (Van Dyck and Baguette 2005; Baguette and Van Dyck 2007). Therefore, more information on movement strategies is also crucial for understanding how dispersal capacities and 
cognitive abilities enable dispersers to make "informed decisions" during the dispersal process and how they affect the connectivity of populations (Vuilleumier and Perrin 2006; Clobert et al. 2009). This information would help building realistic models for the dynamics of metapopulation networks and designing appropriate conservation strategies for fragmented populations (Ims and Yoccoz 1997).

Finally, with information about dispersal strategies the application of justified species-specific definitions of dispersal will be possible. This will allow for a quantification of SBD-rates, that are comparable between studies, which currently represents a big handicap of comparative dispersal studies (Clutton-Brock and Lukas 2012; Le Galliard et al. 2012).

\section{PROXIMATE MECHANISMS OF NATAL DISPERSAL}

Emigration decisions of grey mouse lemurs seem to be condition-dependent, which probably serves to increase the probability of dispersal success. At the very basic level, emigration is cued by the progression in development and growth (Chapter 2), which can be inferred from body mass for subadult grey mouse lemurs. Already emigrated individuals usually had a body mass above $35 \mathrm{~g}$. This threshold of minimum required body mass or size might be a common feature in species, which show a positive association between dispersal propensity and physical condition (Nunes and Holekamp 1996; Edelman 2011). Whether this threshold is stable within and between years still needs to be determined for grey mouse lemurs, but once the critical developmental state is surpassed, other cues determine the exact timing of dispersal. By gathering this information about basic proximate mechanisms, researchers will be able to decide which animals should be targeted for the study of dispersal behaviour.

Since male grey mouse lemurs almost exclusively emigrate (Radespiel et al. 2003; Eberle and Kappeler 2004b; Fredsted et al. 2004), it seems unlikely that males can be distinguished into different classes of males or different dispersal phenotypes (Clobert et al. 2009), though this consideration does not exclude the possibility that variation in intrinsic dispersal motivations exists, which could be the result of additive genetic effects for example (Roff and Fairbairn 2001). Still, emigration seems to be the default behaviour of the majority of subadult males and dispersal is only prevented or delayed by different conditional aspects, as indicated by the body mass threshold, but also by the two observed cases of unsuccessful dispersal described in Chapter 1. This point of view seems to contrast with some aspects of current theoretical concepts of dispersal behaviour, which constitute, on 
the one hand, the existence of specific dispersal phenotypes but attribute, on the other hand, enormous flexibility and plasticity to the dispersal process of an individual (Bonte and De La Peña 2009; Clobert et al. 2009). Though it is usually acknowledged that dispersal is voluntary and not forced in most species (Greenwood 1980; Lawson Handley and Perrin 2007), researchers often do not consider the fact that emigration decisions in species with strong SBD do not necessarily need proximate cueing of "who" but rather of "when" (Nunes et al. 1998). Moreover, elements of condition-dependent dispersal can be ordered in a hierarchical manner with rather inflexible components such as the identified threshold at the basis (Ronce et al. 2001). These inflexible aspects are probably crucial, since they prevent the dispersal of individuals which are not (yet) ready to disperse. Therefore, if the members of one sex have an intrinsically high motivation to disperse, interpreting individual decisions to disperse or to remain philopatric in regard to evolutionary aspects has to be done with great caution, especially if these cases cannot be judged on the basis of detailed behavioural observations. Philopatry can be the result of unsuccessful dispersal or of unfavourable conditions that prevented dispersal and have, thus, only limited diagnostic value for the evolutionary causes of SBD.

In contrast to dispersal probability, dispersal distances were not related to body mass or body condition. For grey mouse lemurs, flexibility of dispersal distances is probably adaptive because of the patchy distribution of populations within continuous forests (Fredsted et al. 2004). Dispersal distances seem to be at least partially determined by genetic factors in some species (Pasinelli et al. 2004; Bitume et al. 2010; Selonen and Hanski 2010), but the expression of these genetic factors depends in turn on population density and social factors. However, studying dispersal distances is usually impeded by the fact that the spatial scale of many studies is not adjusted to the dispersal capacities of a species. As a consequence, low distances are detected more frequently, which creates a biased image of the true dispersal kernel (distribution of dispersal distances) of a species (Koenig et al. 1996; Clobert et al. 2009). If studies were conducted at a spatial scale adjusted to the dispersal capacities of a species, the combination of demographic and pedigree data could offer insights about heritability of distances and the influence of external factors. Moreover, since in some species travelled dispersal distances seems to create a fitness trade-off, for example in fecundity, a comparison of detailed pedigrees could help quantifying whether costs and benefits differ over the dispersal kernel (Roff and Fairbairn 2001; Pasinelli et al. 2004). 


\section{SEX-SPECIFIC BEHAVIOURAL DIFFERENCES}

Since changes of the internal state are not visible, studying them is problematic not only in the framework of dispersal. In this situation, observed behavioural sequences can be used to make inferences about how the individual motivational state varies in relation to different covariates. In Chapter 3 this strategy was exemplified using hidden mixed Markov models for sequences of feeding behaviour. Changes in behavioural consistency between the feeding and non-feeding state were dependent on body mass and showed a general sexdifference for subadult grey mouse lemurs. In the current state, the model presents only a first step and allows no conclusion concerning the internal motivational changes during dispersal at this point. Nevertheless, modelling could be used to identify the existence of dispersal related behavioural syndromes, to relate motivational changes in individuals to physiological changes or to quantify the effect of unfamiliarity on feeding performance or movement decisions (McFarland 1999; Clobert et al. 2009). If future studies of dispersal behaviour of grey mouse lemurs focused on individuals that have reached the minimum required body mass for dispersal (Chapter 2 ), modelling behavioural sequences and also locational data collected from the pre- to post-dispersal phase could identify which kind of behavioural changes occur in the course of dispersal. Once extended, the model could be used to study what proximate factors affect the dispersal behaviour or help identifying behavioural changes that manifest themselves in the continuity rather than in quantity (Réale et al. 2005; Cote et al. 2012). Therefore, while the application described in this thesis represents rather a depiction of the possibilities and advantages of hidden mixed Markov models for the study of behaviour, the various options for extending the basic model make it an interesting tool for biologists from many fields.

\section{CONSEQUENCES OF SEX-SPECIFIC DISPERSAL STRATEGIES}

The consequences of sex-specific dispersal propensities were evaluated in Chapter 4. This part of the thesis used trapping and pedigree data collected over a 10-year period to test for the possible presence of inbreeding depression, inbreeding risk and inbreeding avoidance mechanisms. Inbreeding was not completely eliminated through dispersal in grey mouse lemurs. Nevertheless, despite the heavily SBD and singularity of dispersal, there was no indication for the presence of an inbreeding depression indexed through reduced survival in the studied population. I suggested that the combination of natal dispersal with other 
factors like promiscuity and high mortality made the occurrence of breeding between relatives an extremely rare event. Whether grey mouse lemurs ensure outbreeding by discriminating mates based on genetic similarity was not accessible, because relatedness estimates of true parents and random mates were both extremely low and not distinguishable.

Until now experimental approaches presented the best solution to target the importance of evolutionary mechanisms of SBD, but they need to be carefully designed, since dispersal can be the result of a combination of factors (Ronce et al. 2001). I consider investigations and results presented in Chapter $\mathbf{4}$ as important for the progress in our general understanding of the evolutionary causes of SBD, because they readdress some of the majorly accepted assumptions about the possibility to interpret individual behaviour in regard to ultimate mechanisms like inbreeding avoidance. Especially, since more and more studies have revealed that occasional inbreeding occurs in species with different degrees of SBD (Duarte et al. 2003; Nielsen et al. 2012; Olson et al. 2012; Waser et al. 2012), studying the consequences of SBD could help us understand in how far dispersal really fulfils the functions of competition and inbreeding avoidance which are usually assigned to it.

Since the arrival of genetic methods the number of studies identifying SBD trends for different species has increased exponentially (Lawson Handley and Perrin 2007). In contrast, analyses of detailed long-term data on individual life histories are only available for a comparably small number of species (e.g. Pasinelli et al. 2004; Armitage et al. 2011; Waser et al. 2012). As a result, assumptions about the evolution of SBD are applied to species without the necessary data-based proof. However, empirical studies usually fail to disentangle their relative importance, also because the different evolutionary causes lead to quite similar predictions (Ronce et al. 2001). For grey mouse lemurs, all available studies indicate a strong male-bias in dispersal tendencies (Radespiel et al. 2003; Fredsted et al. 2004; Gligor et al. 2009). Hence, natal dispersal seems to be the default behaviour for males, which could have already been manifested within the ancestors of mouse lemur species. However, inbreeding risk between relatives was not eliminated as a consequence of male-biased dispersal alone, but in combination with other factors made inbreeding between close relatives an extremely unlikely event. In grey mouse lemurs, the strategy is embedded in a system characterized by a high turnover of individuals (Kraus et al. 2008). Therefore, it seems unlikely that inbreeding avoidance was the sole factor for the evolution of heavily SBD in grey mouse lemurs. This 
stands in contrast to early essays on the evolution of SBD, which assumed that a strong SBD evolves as a response to inbreeding risk (Pusey 1987).

Still, the study of different extents of SBD can be very informative, if considered from a different point of view. A way of targeting SBD evolution could be to focus on what allows or favours dispersal tendencies to become a fixed trait of one sex and under which conditions this is not favoured or prevented. Clutton-Brock and Lukas (2012) initiated such an approach through their meta-analysis of female dispersal in group-living mammals. They analysed the occurrence of female dispersal in relation to tenure length of fathers and concluded that intergenerational overlap of reproductive activity can promote an increase of female dispersal rates in polygynous mammals (Lukas and Clutton-Brock 2011; Clutton-Brock and Lukas 2012). Therefore, a strong bias of dispersal propensities could be either seen as a result of selection pressures promoting its fixation or alternatively as the result of a lack of counter-selection preventing it (Kawecki 2000). However, to get a better understanding of SBD, we need more information about the genetic factors involved in dispersal and if these elements are homologous or convergent mechanisms in different species. Possibly, more equal dispersal rates are the result of balancing selection. Studies on insects show how balancing selection is responsible for maintaining genetic and phenotypic diversity in the context of dispersal (Roff and Fairbairn 2001; Haag et al. 2005). Based on this observation, the flexibility of some species in the decision to disperse or not should make them susceptible to experimental manipulation, while species with strong SBD should be relatively resilient to experimental manipulation. For example, experimental removal of the members of one sex does often not inhibit the emigration of the remaining members of the respective sex (Dobson 1982).

Another insightful approach to study patterns of SBD could be between-species comparisons within a smaller taxonomic group as has been done for arvicoline rodents (Le Galliard et al. 2012). However, a comparable amount of data for species of the same family (or subfamily) is seldom available. Alternatively, researchers could gain insights about function and the consequences of dispersal by comparing natural and disturbed populations of the same species. A comparison could inform us not only about the flexibility and limitations of dispersal strategies but also about the robustness of dispersal trends, when the consequences change because the balance created by dispersal is affected. Nevertheless, as long as most of the basic information about the dispersal process is missing, 
the study of the evolutionary causes of dispersal will probably stagnate and make no real progress, because theoretical concepts cannot be developed. Only by collecting data on details on dispersal behaviour, we will be able to annex proximate and ultimate mechanisms to finally understand what caused the existence of SBD, especially since more and more studies reveal that SBD is also a common feature in reptiles, amphibians and fish (e.g. Tucker et al. 1998; Hutchings and Gerber 2002; Austin et al. 2003; Lampert et al. 2003; Dubey et al. 2008). By acknowledging that SBD trends exist in a continuum from no to weak to heavy $\mathrm{SBD}$, we could shift our attention from the question of whether a sex-bias exists to the question what determines the different degrees of SBD and why dispersal remains more flexible in some species.

\section{CONCLUSIONS}

This thesis added not only to existing knowledge about heavily male-biased natal dispersal tendencies in grey mouse lemurs (Radespiel et al. 2003; Fredsted et al. 2004) but also to our general understanding of SBD by studying the dispersal process of this species and the consequences of male-biased natal dispersal. I showed that the dispersal strategies used by a species can be very uniform with deterministic basic proximate cues controlling the emigration timing. Therefore, it seems that SBD has experienced canalizing selection, making dispersal an almost fixed life history trait of male grey mouse lemurs. These findings appear to stand in contrast to suggestions of previously published work indicating that dispersal behaviour would be characterized by a high plasticity and flexibility (Bonte and De La Peña 2009; Clobert et al. 2009). Embedded in a system which is characterized by a fast life history, high population turnover rates (Kraus et al. 2008) and high degree of promiscuity (Eberle and Kappeler 2002), the relative inflexibility of the dispersal regime still seems to create enough mixture within and between populations to eliminate evolutionary problems such as inbreeding risk and to ensure the persistence of populations.

However, one aspect that became obvious in almost all chapters of this thesis was the realization that the study of dispersal suffers from several specific problems and draw backs, which are mainly a consequence of the lack of knowledge about dispersal strategies. First, the spatial scale of most studies is not adjusted to dispersal capacities. This impedes the study of many aspects related to dispersal, for example the effects of population density on the distribution of dispersal distances or analyses of survival. As another consequence, 
considerations of dispersal costs remain often of theoretical nature for most species, because the insufficient spatial scale reduces or biases the number of complete life history records for dispersers.

Second, most studies apply rather arbitrary dispersal definitions. By knowing relocation strategies, unified species-specific definitions could be constituted which would facilitate between-study comparisons and the inference of representative dispersal rates. Both factors can cause additional variation, making comparisons between and within species difficult. Finally, the invention of genetic methods and the possibility to use increased computing capacities have caused an unbalanced progress in the different areas of dispersal research. However, despite the difficulty to study dispersal movements it will be impossible to circumvent the necessity to collect more data on this aspect of dispersal, if we want to learn more about the adaptive value of dispersal and develop theoretical frameworks on its evolution.

\section{OUTLOOK}

The study of SBD requires more than just identifying its existence for species with different mating systems if we want to understand how one solution, namely SBD, can help solve different ultimate problems. The rapid improvements of tracking devices will hopefully soon lead to a revolution for the study of dispersal strategies and distances, which remain aspects of dispersal receiving only little attention. Possibly, many observations of dispersal have been documented, but because of their anecdotal character remain unpublished. Therefore, I would suggest the establishment of a public platform focussing on the collection of information on dispersal strategies or the integration of a special section into already existing platforms (such as "MOVEBANK") as an initial step to incite the publication of such observations and, subsequently, the study of dispersal movements.

Concerning the proximate and evolutionary causes of dispersal, I would suggest a reconsideration of our current understanding of SBD, which should focus on the variability in the extent of SBD and not on its mere existence, and what this means for the flexibility concerning the decision dispersal yes/no. I acknowledge that dispersal is proximately cued by a variety of factors, but based on my observations I would also like to argue that a heavy sex-bias in dispersal rates can indicate an intrinsically high motivation of an individual to disperse. Therefore, I would advise a careful consideration of the meaning of individual 


\section{GENERAL DISCUSSION}

behaviour in regard to ultimate mechanisms, especially for species with heavily SBD. Finally, we probably need to acknowledge that past evolutionary pressures might not be measurable anymore in recent species. In this respect it would be interesting to know, which species are currently under selective pressure acting to modify current dispersal regimes and whether these cases represent the whole continuum of SBD trends. Quantifying the consequences of a dispersal regime for the presence or absence of competition and inbreeding avoidance could present a strategy to detect such differences. 


\section{SUMMARY}

Dispersal, the permanent relocation or change of social group of an organism, is an almost ubiquitous phenomenon among taxa. Sex-biased dispersal (SBD) propensities and/or distances are of one of the most common general dispersal patterns. With the invention of genetic tools the number of studies identifying dispersal trends has increased exponentially. Still, aspects of the dispersal process remain unknown for most species, impeding not only our understanding of the evolution of SBD, but also knowledge about the connectivity of populations, which finally determines the dynamics of populations. Consequently, many of the assumptions about the costs induced by dispersal like increased mortality remain of theoretical nature. In this thesis, I investigated proximate aspects of the dispersal process and consequences of sex-biased natal dispersal of a small, solitary primate, the grey mouse lemur (Microcebus murinus) by means of behavioural observations and radio-tracking of 90 subadult grey mouse lemurs as well as capture-mark-recapture and genetic analyses. I found that male grey mouse lemurs have a uniform movement strategy during dispersal with highly directed movements and spatially very concentrated explorative activity. The length of the whole dispersal process varied, because individuals varied in the period during which they commuted between natal home range and prospected sites. This observation indicated that unfamiliarity with the habitat during immigration presented the biggest challenge during dispersal for grey mouse lemurs in terms of dispersal-related costs. This assumption was further supported by another finding, derived from capture-mark-recapture and genetic data collected for a long-term study. Prior to emigration, grey mouse lemurs needed to accomplish a minimum degree of development and growth. This minimum level of maturity required for dispersal served probably as a preparation for the negative effects of dispersal on the energy balance. Such condition-dependent dispersal strategies seem to be very common, since they allow for flexibility in dispersal behaviour such as the exact timing of emigration, which probably helps to increase the success probability of dispersal. Possibly, such preparations freed dispersal distances from constraints of physical condition in grey mouse lemurs, which was neither determined by body mass nor by body condition. Once the critical threshold was overcome other factors determined the exact timing of emigration. Which proximate factors, remains to be determined, but promising directions for future investigations represent the study of personality differences or physiological changes. In this 
thesis, I also introduced an approach that could be used to detect behavioural changes during dispersal and how these changes are related to factors such as personality of hormonal changes. The approach was exemplified by modelling feeding sequences of subadult males and females. Finally, I used a ten-generation capture-mark-recapture and genetic data set collected for a long-term study to evaluate, whether male-biased natal dispersal effectively eliminated the risk of inbreeding, which is one of the factors which is generally accepted to play an important role in the evolution of SBD. No signs for inbreeding depression in terms of survival could be detected and natal dispersal decreased inbreeding risk substantially. However, only in combination with demographic (such as mortality) and behavioural factors (roaming of males during mating season and promiscuity) was inbreeding risk reduced to such an extent, that additional dispersal of either males or females becomes unnecessary. This situation probably allowed SBD propensities to become and stay an almost fixed event in the life history of male grey mouse lemurs, which is rather insensible to external cues and deterministic concerning the emigration decision, but allows for some flexibility in the timing of dispersal, which enables males to improve their prospects of successful dispersal. However, which factors caused the evolution of SBD in grey mouse lemurs remains to be determined. 


\section{ZUSAMMENFASSUNG}

Abwanderung, definiert als eine permanente Verlagerung von Streifgebieten, Territorien oder als ein permanenter Wechsel zu neuen sozialen Gruppen, ist ein nahezu omnipräsentes Phänomen. Ein weit verbreitetes Muster im Tierreich sind geschlechtsspezifische Abwanderungsraten (SBD) und/oder -distanzen. Mit der Entdeckung und dem Fortschritt genetischer Analysemethoden hat sich die Anzahl der Studien, die geschlechtsspezifische Abwanderungsraten in den verschiedensten Tierarten untersuchen, exponentiell vervielfacht. Im Gegenzug dazu bleiben Aspekte wie der Abwanderungsprozess weitgehend unerforscht. Dieses Ungleichgewicht in unserem Wissensstand über SBD limitiert nicht nur unser Verständnis zur Evolution von SBD, sondern auch unser Wissen über die Dynamiken und Prozesse, die die verschiedenen Populationen einer Art verbinden. Dementsprechend bleiben viele essentielle Annahmen zu möglichen Kosten und Bedrohungen während des Abwanderns von eher theoretischer Natur.

In der vorliegenden Arbeit habe ich proximate Aspekte des Abwanderungsprozesses für einen kleinen, solitär aktiven Primaten, den grauen Mausmaki (Microcebus murinus), dokumentiert, sowie die Konsequenzen von stark einseitigen Abwanderungstendenzen junger Männchen untersucht mittels Fokustierbeobachtungen und Telemetrie, sowie FangWiderfang-Daten und genetischer Analysen untersucht. Die dokumentierte Abwanderungsstrategie war durch uniforme, stark gerichtete Bewegungsmuster gekennzeichnet und Erkundungsaktivitäten waren auf einen sehr limitierten Raum beschränkt. Die Dauer des Prozesses variierte zwischen Individuen, da diese unterschiedlich stark und lange zwischen alten und neuen Streifgebieten pendelten, bevor sie endgültig ihr altes Streifgebiet verließen. Diese Beobachtung deutet an, dass die Einwanderungsphase den schwierigsten Teil des Abwanderungsprozesses für graue Mausmakis in Bezug auf die abwanderungsbezogenen Kosten darstellt. Diese Annahme wurde durch einen weiteren Befund unterstützt, der auf Fang-Wiederfang- und genetischen Daten basierte. Für abwandernde graue Mausmakis gibt es scheinbar eine minimal erforderliche Körpergröße für Abwanderer. Dieses Minimalmaß an Entwicklungsreife scheint als Absicherung gegen die mit Abwanderung assoziierten energetischen Kosten zu dienen. Abwanderungsstrategien, die einen Abgleich innerer mit äußeren Bedingungen erlauben, sogenannte konditionsabhängige Strategien, scheinen weitverbreitet zu sein, da sie es einem Individuum 
erlauben, sein Abwanderungsverhalten und damit die Aussicht auf Erfolg zu optimieren. Für graue Mausmakis führt diese Strategie, eine gewisse physische Kondition zu erreichen, anscheinend auch mit sich, dass sie weniger eingeschränkt sind bezüglich der zurückgelegten Abwanderungsdistanzen. Diese Annahme beruht auf der Tatsache, dass weder Körpergewicht, noch -kondition einen Zusammenhang mit Abwanderungsdistanzen aufwiesen. Nach Erreichen des kritischen Wertes an körperlicher Entwicklung bestimmen andere Faktoren den genauen Zeitpunkt für die Emigration. Welche Faktoren dies im Einzelnen sind, muss in weiteren Projekten untersucht werden. Persönlichkeit/Temperament oder physiologische Faktoren wie z.B. Hormone scheinen vielversprechende Ansatzpunkte für weitere Untersuchungen zu sein. In Bezug darauf, beinhaltet diese Arbeit einen Modellansatz, der es ermöglicht, den Einfluss verschiedenster Faktoren auf Verhaltensequenzen zu untersuchen, was anhand von beobachtetem Fressverhalten für Männchen und Weibchen exemplarisch dargestellt wurde. Dieser Ansatz könnte in Zukunft dazu genutzt werden, Veränderungen im Verhalten im Laufe des Abwanderungsprozesses zu untersuchen.

Der letzte Teil der vorliegenden Arbeit evaluiert die Konsequenzen von stark einseitigen Abwanderungstendenzen junger Männchen für den Fortbestand von Inzuchtrisiko, einem der meistgenannten ultimaten Mechanismen für die Evolution von SBD. Es konnte kein Hinweis auf Inzuchtdepression ausgemacht werden und SBD verringerte das Inzuchtrisko bereits beträchtlich. Allerdings war das tatsächliche Inzuchtrisiko noch weit geringer, da die Wirkung von Abwanderung zusätzlich durch demografische (z.B. Mortalität) und Verhaltensmechanismen (z.B. die weiträumige Suche nach Paarungspartner durch Männchen) ergänzt wurde. Als Konsequenz daraus scheint jede weitere Abwanderungsaktivität von Männchen oder Weibchen überflüssig zu sein. Diese Situation erlaubt es scheinbar auch, dass geschlechtsspezifische Abwanderungstendenzen ein fester Teil männlicher „life histories" geworden sind und als solcher bestehen. Einzig der Abwanderungsprozess scheint einen gewissen Grad an Flexibilität in Bezug auf den Zeitpunkt des Abwanderns zu erlauben, was es grauen Mausmakimännchen wahrscheinlich ermöglicht, ihre Erfolgsaussichten zu erhöhen. Indes, was die genauen Ursachen für die Evolution von SBD in grauen Mausmakis waren, bleibt zu ermitteln. 


\section{ACKNOWLEDGEMENTS}

First and most importantly, l'd like to express my gratitude to my supervisor Peter Kappeler, who gave me the chance and the position to do this project. Peter was one of the few people who always appreciated the importance of studying dispersal mechanisms, which I found very encouraging. I would also like to thank him for his openness to ideas and for lending his seemingly infinite experience.

I would also like to thank Eckhard Heymann for his effort and support as a referee of this dissertation. He was also a valued discussion partner during my dissertation, offering helpful advice and ideas.

Next, I would like to thank Manfred Eberle for his infectious enthusiasm for mouse lemurs and his support. This thesis built also to a large extent on his diligent data collection.

Special thanks go to Roland Langrock for lending his expertise for the analysis of the behavioural data. The enthusiasm and creativity that he contributed to this project were very much welcomed and appreciated.

In Madagascar, I thank M. Daniel Rakotondravony, and the following institutions: Département de Biologie Animale d'Université d'Antananarivo, the Commission Tripartite and the CAFF of the Direction des Eaux et Forêts and the CNFEREF Morondava for their kind authorization and support of this study. I sincerely thank Léonard Razafimanantsoa and Rodin Rasoloarison for their marvellous logistic support in the field. Misaotra betsaka ny nàmana Bruno Tsiverimana, Remy de Ampataka and Tiana Andrianjanahary for their imperturbable good mood and invaluable, indispensable assistance in countless trapping sessions. Many thanks go to the whole Equipe Kirindy and to Elise Huchard and Anne-Claire Collet for help with radio-tracking, trapping sessions and many other things.

This project was generously financed by the support from the German Excellence Initiative (Courant Centre "Evolution of Social Behaviour") and Deutsches Primatenzentrum Göttingen. 


\section{ACKNOWLEDGEMENTS}

Thanks to the anonymous reviewers, who provided helpful comments on earlier versions of unpublished manuscripts.

Special thanks go to my colleagues of the Behavioral Ecology \& Sociobiology Unit of the DPZ and the Department of Sociobiology/Anthropology of the University of Göttingen, present and former ones, who accompanied me through this thesis, for being a valuable source of discussions and feedback, but also for being more than just colleagues. In particular I would like to thank the following people: my office mates, Matthias Markolf and Lennart Pyritz, my fellow sufferers, Mia-Lana Lührs and Melanie Dammhahn, who were an important support during the final phase of this thesis, and finally Melanie Dammhahn, Elise Huchard and Cornelia Krauss, who spent their time listening to and discussing ideas. Working with them was instructive, motivating and inspiring. Their support was in many ways amazing and encouraging. Thanks to Marie Dittmann for her help in the lab with genotyping mouse lemurs. At last, I gratefully acknowledge the logistic support by Christina Oberdieck, Anja Engelke, Gunhilde Wilz, Henning Lahmann and Ulrike Walbaum.

Finally, I want to express my deep gratitude for the unlimited and unearthly support, which I received from my family and friends. Words cannot express what that meant to me. 


\section{REFERENCES}

Acevedo-Whitehouse K, Gulland F, Greig D, Amos W (2003) Inbreeding: disease susceptibility in California sea lions. Nature 422: 35-35

Alberts SC, Altmann J (1995a) Balancing costs and opportunities: dispersal in male baboons. Am Nat 145: 279-306

Alberts SC, Altmann J (1995b) Preparation and activation: determinants of age at reproductive maturity in male baboons. Behav Ecol Sociobiol 36: 397-406

Altman R (2007) Mixed hidden Markov models: an extension of the hidden Markov model to the longitudinal data setting. JASA 102: 201-210

Amos W, Worthington Wilmer J, Fullard K, Burg TM, Croxall JP, Bloch D, Coulson T (2001) The influence of parental relatedness on reproductive success. Proc R Soc B 268: 20212027

Andreassen HP, Stenseth NC, Ims RA (2002) Dispersal behaviour and population dynamics of vertebrates. In: Bullock JM, Kenward RE, Hails RS (eds) Dispersal Ecology. Blackwell Publishing Ltd, Oxford, pp 237-256

Aparicio JM, Cordero PJ, Veiga JP (2001) A test of the hypothesis of mate choice based on heterozygosity in the spotless starling. Anim Behav 62: 1001-1006

Aparicio JM, Ortego J, Cordero PJ (2006) What should we weigh to estimate heterozygosity, alleles or loci? Mol Ecol 15: 4659-4665

Armitage KB (1991) Social and population dynamics of yellow-bellied marmots: results from long-term research. Annu Rev Ecol Syst 22: 379-407

Armitage KB, Vuren DHV, Ozgul A, Oli MK (2011) Proximate causes of natal dispersal in female yellow-bellied marmots, Marmota flaviventris. Ecology 92: 218-227

Austin JD, Dávila JA, Lougheed SC, Boag PT (2003) Genetic evidence for female-biased dispersal in the bullfrog, Rana catesbeiana (Ranidae). Mol Ecol 12: 3165-3172

Baars MA (1979) Patterns of movement of radioactive carabid beetles. Oecol 44: 125-140

Baguette M, Van Dyck H (2007) Landscape connectivity and animal behavior: functional grain as a key determinant for dispersal. Landsc Ecol 22: 1117-1129

Baker RR (1969) The evolution of the migratory habit in butterflies. J Anim Ecol 38: 703-746

Balloux F, Amos W, Coulson T (2004) Does heterozygosity estimate inbreeding in real populations? Mol Ecol 13: 3021-3031

Banachewicz K, Lucas, A., Vaart A (2008) Modelling portfolio defaults using hidden Markov models with covariates. Economet J 11: 155-171

Barbraud C, Johnson AR, Bertault G (2003) Phenotypic correlates of post-fledging dispersal in a population of greater flamingos: the importance of body condition. J Anim Ecol 72: 246-257

Bartolucci F, Lupparelli M, Montanari GE (2009) Latent Markov model for longitudinal binary data: an application to the performance evaluation of nursing homes. Ann Appl Stat 3: 611-636

Barton KA, Phillips BL, Morales JM, Travis JMJ (2009) The evolution of an 'intelligent' dispersal strategy: biased, correlated random walks in patchy landscapes. Oikos 118: 309-319

Barton NH (2001) The evolutionary consequences of gene flow and local adaptation: future approaches. In: Clobert J, Danchin E, Dhondt AA, Nichols JD (eds) Dispersal. Oxford University Press, New York, pp 329-340 
Bates D, Maechler M, Bolker B (2011) Ime4: linear mixed-effects models using S4 classes, R package version 0.999375-42.

Bearder SK (1987) Lorises, bushbabies, and tarsiers: diverse societies in solitary foragers. In: Smutts BB, Cheney DL, Seyfarth RM, Wrangham RW, Struhsaker TT (eds) Primate societies. The University of Chicago Press, Chicago, pp 11-33

Belthoff JR, Dufty JAM (1998) Corticosterone, body condition and locomotor activity: a model for dispersal in screech-owls. Anim Behav 55: 405-415

Bitume EV, Bonte D, Magalhāes S, San Martin G, Van Dongen S, Bach F, Anderson JM, Olivieri I, Nieberding CM (2010) Heritability and artificial selection on ambulatory dispersal distance in Tetranychus urticae: effects of density and maternal effects. PLoS ONE 6: e26927

Blouin SF, Blouin M (1988) Inbreeding avoidance behaviors. Trends Ecol Evol 3: 230-233

Boinski S, Kauffman L, Ehmke E, Schet S, Vreedzaam A (2005) Dispersal patterns among three species of squirrel monkeys (Saimiri oerstedii, S. boliviensis and S. sciureus): I. Divergent costs and benefits. Behaviour 142: 525-632

Bollinger EK, Harper SJ, Barrett GW (1993) Inbreeding avoidance increases dispersal movements of the meadow vole. Ecology 74: 1153-1156

Bonte D, De La Peña E (2009) Evolution of body condition-dependent dispersal in metapopulations. J Evol Biol 22: 1242-1251

Bonte D, Van Dyck H, Bullock JM, Coulon A, Delgado M, Gibbs M, Lehouck V, Matthysen E, Mustin K, Saastamoinen M, Schtickzelle N, Stevens VM, Vandewoestijne S, Baguette M, Barton K, Benton TG, Chaput-Bardy A, Clobert J, Dytham C, Hovestadt T, Meier CM, Palmer SCF, Turlure C, Travis JMJ (2012) Costs of dispersal. Biol Rev 87: 290-312

Bowler DE, Benton TG (2005) Causes and consequences of animal dispersal strategies: relating individual behaviour to spatial dynamics. Biol Rev 80: 205-225

Bowman J, Jaeger JAG, Fahrig L (2002) Dispersal distance of mammals is proportional to home range size. Ecology 83: 2049-2055

Breed GA, Jonsen ID, Myers RA, Bowen WD, Leonard ML (2009) Sex-specific, seasonal foraging tactics of adult grey seals (Halichoerus grypus) revealed by state-space analysis. Ecology 90: 3209-3221

Brockelman WY, Reichard U, Treesucon U, Raemaekers JJ (1998) Dispersal, pair formation and social structure in gibbons (Hylobates lar). Behav Ecol Sociobiol 42: 329-339

Brouwer L, Komdeur J, Richardson DS (2007) Heterozygosity-fitness correlations in a bottlenecked island species: a case study on the Seychelles warbler. Mol Ecol 16: 3134-3144

Brown JL (1997) A theory of mate choice based on heterozygosity. Behav Ecol 8: 60-65

Buckland ST, Newman KB, Thomas L, Koesters NB (2004) State-space models for the dynamics of wild animal populations. Ecol Model 171: 157-175

Burland TM, Barratt EM, Nichols RA, Racey PA (2001) Mating patterns, relatedness and the basis of natal philopatry in the brown long-eared bat, Plecotus auritus. Mol Ecol 10: 1309-1321

Burnham KP, Anderson DR (2002) Model selection and multimodel inference: a practical information-theoretic approach. Springer-Verlag, New York

Burns CE (2005) Behavioral ecology of disturbed landscapes: the response of territorial animals to relocation. Behav Ecol 16: 898-905

Byrne RW (1979) Memory for urban geography. Q J Exp Psychol 31: 147-154

Caraco T (1980) On foraging time allocation in a stochastic environment. Ecology 61: 119-128 
Castanet J, Croci S, Aujard F, Perret M, Cubo J, de Margerie E (2004) Lines of arrested growth in bone and age estimation in a small primate: Microcebus murinus. J Zool 263: 31-39

Charlesworth B, Charlesworth D (1999) The genetic basis of inbreeding depression. Genet Res 74: 329-340

Charlesworth D, Charlesworth B (1987) Inbreeding depression and its evolutionary consequences. Annu Rev Ecol Syst 18: 237-268

Charlesworth D, Willis JH (2009) The genetics of inbreeding depression. Nat Rev Genet 10: 783-796

Charnov EL (1976) Optimal foraging, the marginal value theorem. Theor Popul Biol 9: 129136

Charpentier M, Setchell JM, Prugnolle F, Wickings EJ, Peignot P, Balloux F, Hossaert-Mckey M (2006) Life history correlates of inbreeding depression in mandrills (Mandrillus sphinx). Mol Ecol 15: 21-28

Charpentier MJE, Widdig A, Alberts SC (2007) Inbreeding depression in non-human primates: a historical review of methods used and empirical data. Am J Primatol 69: 1370-1386

Clark JS (2005) Why environmental scientists are becoming Bayesians. Ecol Lett 8: 2-14

Clobert J, Ims RA, Rousset F (2004) Causes, mechanisms and consequences of dispersal. In: Hanski I, Gaggiotti O (eds) Ecology, genetics and evolution of metapopulations. Elsevier Academic Press, Burlington, pp 307-335

Clobert J, Le Galliard J-F, Cote J, Meylan S, Massot M (2009) Informed dispersal, heterogeneity in animal dispersal syndromes and the dynamics of spatially structured populations. Ecol Lett 12: 197-209

Clutton-Brock T, McAuliffe K (2009) Female mate choice in mammals. Q Rev Biol 84: 3-27

Clutton-Brock TH (1989) Female transfer and inbreeding avoidance in social mammals. Nature 337: 70-72

Clutton-Brock TH, Lukas D (2012) The evolution of social philopatry and dispersal in female mammals. Mol Ecol 21: 472-492

Cohas A, Bonenfant C, Kempenaers B, Allainé D (2009) Age-specific effect of heterozygosity on survival in alpine marmots, Marmota marmota. Mol Ecol 18: 1491-1503

Coltman DW, Bowen WD, Wright JM (1998) Birth weight and neonatal survival of harbour seal pups are positively correlated with genetic variation measured by microsatellites. Proc R Soc B 265: 803-809

Coltman DW, Pilkington JG, Smith JA, Pemberton JM (1999) Parasite-mediated selection against inbred Soay sheep in a free-living, island population. Evolution 53: 1259-1267

Coltman DW, Slate J (2003) Microsatellite measures of inbreeding: a meta-analysis. Evolution 57: 971-983

Conradt L, Roper TJ (2006) Nonrandom movement behavior at habitat boundaries in two butterfly species: implications for dispersal. Ecology 87: 125-132

Conradt L, Zollner PA, Roper TJ, Frank K, Thomas CD (2003) Foray search: an effective systematic dispersal strategy in fragmented landscapes. Am Nat 161: 905-915

Constable JL, Ashley MV, Goodall J, Pusey AE (2001) Noninvasive paternity assignment in Gombe chimpanzees. Mol Ecol 10: 1279-1300

Cooney R, Bennett NC (2000) Inbreeding avoidance and reproductive skew in a cooperative mammal. Proc R Soc B 267: 801-806

Cormack RM (1964) Estimates of survival from the sighting of marked animals. Biometrika 51: 429-438 
Cote J, Clobert J, Brodin T, Fogarty S, Sih A (2012) Personality-dependent dispersal: characterization, ontogeny and consequences for spatially structured populations. Philos Trans R Soc B 365: 4065-4076

Coulson TN, Pemberton JM, Albon SD, Beaumont M, Marshall TC, J S, Guinness FE, CluttonBrock TH (1998) Microsatellites reveal heterosis in red deer. Proc R Soc B 265: 489495

Dammhahn M (2012) Are personality differences in a small iteroparous mammal maintained by a life-history trade-off? Proc R Soc B 279: 2645-2651

Dammhahn M, Kappeler PM (2008a) Comparative feeding ecology of sympatric Microcebus berthae and M. murinus. Int J Primatol 29: 1567-1589

Dammhahn M, Kappeler PM (2008b) Small-scale coexistence of two mouse lemur species (Microcebus berthae and M. murinus) within a homogeneous competitive environment. Oecologica 157: 473-483

Dammhahn M, Kappeler PM (2009) Females go where the food is: does the socio-ecological model explain variation in social organisation of solitary foragers? Behav Ecol Sociobiol 63: 939-952

Dammhahn M, Kappeler PM (2010) Scramble or contest competition over food in solitarily foraging mouse lemurs (Microcebus spp.): new insights from stable isotopes. Am J Phys Anthropol 141: 181-189

Dammhahn M, Kappeler PM (in press) Seasonal patterns and energy strategies of co-existing female Microcebus berthae and M. murinus (Cheirogaleidae). In: Master J, Gamba M, Génin $\mathrm{F}$ (eds) Developments in primatology: leaping ahead: advances in prosimian biology. Springer, New York

Danchin E, Heg D, Doligez B (2001) Public information and breeding habitat selection. In: Clobert J, Danchin E, Dhondt AA, Nichols JD (eds) Dispersal. Oxford University Press, New York, pp 243-258

De Sousa MBC, Albuquerque ACSR, Yamamoto ME, Araújo A, Arruda MF (2009) Emigration as a reproductive strategy of the common marmoset (Callithrix jacchus). In: Davis LC, Ford SM, Porter LM (eds) The smallest anthropoids: the marmoset/callimico radiation. Springer, New York, pp 167-182

Del Mar Delgado M, Penteriani V, Nams VO, Campioni L (2009) Changes of movement patterns from early dispersal to settlement. Behav Ecol Sociobiol 64: 35-43

Delibes M, Ferreras P, Gaona P (2001) Attractive sinks, or how individual behavioural decisions determine source-sink dynamics. Ecol Lett 4: 401-403

Diehl WJ, Koehn RK (1985) Multiple-locus heterozygosity, mortality, and growth in a cohort of Mytilus edulis. Mar Biol 88: 265-271

Dingemanse NJ, Both C, Van Noordwijk AJ, Rutten AL, Drent PJ (2003) Natal dispersal and personalities in great tits (Parus major). Proc R Soc B 270: 741-747

Dobson FS (1982) Competition for mates and predominant juvenile male dispersal in mammals. Anim Behav 30: 1183-1192

Downhower JF, Armitage KB (1981) Dispersal of yearling yellow-bellied marmots (Marmota flaviventris). Anim Behav 29: 1064-1069

Duarte LC, Bouteiller C, Fontanillas P, Petit E, Perrin N (2003) Inbreeding in the greater white-toothed shrew, Crocidura russula. Evolution 57: 638-645

Dubey S, Brown GP, Madsen T, Shine R (2008) Male-biased dispersal in a tropical Australian snake (Stegonotus cucullatus, Colubridae). Mol Ecol 17: 3506-3514 
Duckworth RA, Badyaev AV (2007) Coupling of dispersal and aggression facilitates the rapid range expansion of a passerine bird. Proceedings of the National Academy of Sciences 104: 15017-15022

Dufty AM, Jr., Belthoff JR (2001) Proximate mechanisms of natal dispersal: the role of body condition and hormones. In: Clobert J, Danchin E, Dhondt AA, Nichols JD (eds) Dispersal. Oxford University Press, New York, pp 217-228

Dufty AM, Jr., Clobert J, Møller AP (2002) Hormones, developmental plasticity and adaptation. Trends Ecol Evol 17: 190-196

Durbin J, Koopman SJ (1997) Monte Carlo maximum likelihood estimation for non-Gaussian state space models. Biometrika 84: 669-684

Durbin R, Eddy S, Krogh A, Mitchison GJ (1998) Biological sequence analysis: probabilistic models of proteins and nucleic acids. Cambridge University Press, Cambridge

Eberle M, Kappeler PM (2002) Mouse lemurs in space and time: a test to the socioecological model. Behav Ecol Sociobiol 51: 131-139

Eberle M, Kappeler PM (2004a) Selected polyandry: female choice and inter-sexual conflict in a small nocturnal solitary primate (Microcebus murinus). Behav Ecol Sociobiol 57: 91-100

Eberle M, Kappeler PM (2004b) Sex in the dark: determinants and consequences of mixed male mating tactics in Microcebus murinus, a small solitary nocturnal primate. Behav Ecol Sociobiol 57: 77-90

Eberle M, Kappeler PM (2006) Family insurance: kin selection and cooperative breeding in a solitary primate (Microcebus murinus). Behav Ecol Sociobiol 60: 582-588

Eberle M, Perret M, Kappeler PM (2007) Sperm competition and optimal timing of matings in Microcebus murinus. Int J Primatol 28: 1267-1278

Edelman AJ (2011) Sex-specific effects of size and condition on timing of natal dispersal in kangaroo rats. Behav Ecol 22: 776-783

Estes-Zumpf WA, Rachlow JL (2009) Natal dispersal by pygmy rabbits (Brachylagus idahoensis). J Mammal 90: 363-372

Fernandez-Duque E (2009) Natal dispersal in monogamous owl monkeys (Aotus azarai) of the Argentinean Chaco. Behaviour 146: 583-606

Fernandez-Duque E, Huntington C (2002) Disappearances of individuals from social groups have implications for understanding natal dispersal in monogamous owl monkeys (Aotus azarai). Am J Primatol 57: 219-225

Fietz J (1999) Mating system of Microcebus murinus. Am J Primatol 48: 127-133

Fisher D, Lambin X, Yletyinen S (2009) Experimental translocation of juvenile water voles in a Scottish lowland metapopulation. Popul Ecol 51: 289-295

Foerster K, Delhey K, Johnsen A, Lifjeld JT, Kempenaers B (2003) Females increase offspring heterozygosity and fitness through extra-pair matings. Nature 425: 714-717

Foerster K, Valcu M, Johnsen A, Kempenaers B (2006) A spatial genetic structure and effects of relatedness on mate choice in a wild bird population. Mol Ecol 15: 4555-4567

Fox CW, Rauter CM (2003) Bet-hedging and the evolution of multiple mating. Evol Ecol Res 5: 273-286

Franke A, Caelli T, Kuzyk G (2006) Prediction of wolf (Canis lupus) kill-sites using hidden Markov models. Ecol Model 197: 237-246

Fredsted T, Pertoldi C, Olesen JM, Eberle M, Kappeler PM (2004) Microgeographic heterogeneity in spatial distribution and mtDNA variability of gray mouse lemurs (Microcebus murinus, Primates: Cheirogaleidae). Behav Ecol Sociobiol 56: 393-403 
Fredsted T, Pertoldi C, Schierup H, Kappeler PM (2005) Microsatellite analyses reveal finescale genetic structure in gray mouse lemurs (Microcebus murinus). Mol Ecol 14: 2363-2372

Fromhage L, Kokko H, Reid JM (2009) Evolution of mate choice for genome-wide heterozygosity. Evolution 63: 684-694

Frühwirth-Schnatter S (2006) Finite mixture and Markov switching models. Springer, New York

Génin F (2003) Female dominance in competition for gum trees in the grey mouse lemur Microcebus murinus. La Terre et la Vie - Revue d'écologie 58: 397-410

Gligor M, Ganzhorn JU, Rakotondravony D, Ramilijaona OR, Razafimahatratra E, Zischler H, Hapke A (2009) Hybridization between mouse lemurs in an ecological transition zone in southern Madagascar. Mol Ecol 18: 520-533

Greenwood PJ (1980) Mating systems, philopatry and dispersal in birds and mammals. Anim Behav 28: 1140-1162

Guillaume F, Perrin N (2006) Joint evolution of dispersal and inbreeding load. Genetics 173: 497-509

Gundersen G, Johannesen E, Andreassen HP, Ims RA (2001) Source-sink dynamics: how sinks affect demography of sources. Ecol Lett 4: 14-21

Haag CR, Saastamoinen M, Marden JH, Hanski I (2005) A candidate locus for variation in dispersal rate in a butterfly metapopulation. Proc R Soc B 272: 2449-2456

Hamilton JD (1989) A new approach to the economic analysis of nonstationary time series and the business cycle. Econometrica 57: 357-384

Hanski I, Peltonen A, Kaski L (1991) Natal dispersal and social dominance in the common shrew Sorex araneus. Oikos 62: 48-58

Hansson L (1992) Small mammal dispersal in pest management and conservation. In: Stenseth NC, Lidicker WZ (eds) Animal dispersal: small mammals as a model. Chapman \& Hall, London, pp 181-198

Hapke A, Eberle M, Zischler H (2003) Isolation of new microsatellite markers and application in four species of mouse lemurs (Microcebus sp.). Mol Ecol 3: 205-208

Harrison RL (1992) Toward a theory of inter-refuge corridor design. Conserv Biol 6: 293-295

Heinz SK, Strand E (2006) Adaptive patch searching strategies in fragmented landscapes. Evol Ecol 20: 113-130

Herrera EA, Salas V, Congdon ER, Corriale MJ, Tang-Martínez Z (2011) Capybara social structure and dispersal patterns: variations on a theme. J Mammal 92: 12-20

Holekamp KE (1986) Proximal causes of natal dispersal in Belding's ground squirrels (Spermophilus beldingi). Ecol Monogr 56: 365-391

Holekamp KE, Sherman PW (1989) Why male ground squirrels disperse: a multilevel analysis explains why only males leave home. Am Sci 77: 232-239

Hollander M, Wolfe DA (1999) Nonparametric statistical methods, 2 edn. John Wiley \& Sons, Inc., New York

Hoset KS, Ferchaud A-L, Dufour F, Mersch D, Cote J, Le Galliard J-F (2011) Natal dispersal correlates with behavioral traits that are not consistent across early life stages. Behav Ecol 22: 176-183

Hothorn T, Bretz F, Westfall P (2008a) Simultaneous inference in general parametric models. Biometrical J 50: 346-363

Hothorn T, Hornik K, van de Wiel MA, Zeileis A (2008b) Implementing a class of permutation tests: the coin package. J Stat Softw 28: 1-23 
Houston Al, McNamara JM (1999) Models of adaptive behaviour. Cambridge University Press, New York

Huchard E, Canale Cl, Le Gros C, Perret M, Henry P-Y, Kappeler PM (2011) Convenience polyandry or convenience polygyny? Costly sex under female control in a promiscuous primate. Proc R Soc B 279: 1371-1379

Huchard E, Knapp LA, Wang J, Raymond M, Cowlishaw G (2010) MHC, mate choice and heterozygote advantage in a wild social primate. Mol Ecol 19: 2545-2561

Humphreys K (1998) The latent Markov chain with multivariate random effects. Socio Meth Res 26: 269-299

Hutchings JA, Gerber L (2002) Sex-biased dispersal in a salmonid fish. Proc R Soc B 269: 24872493

Ims RA, Hjermann D $\varnothing$ (2001) Condition-dependent dispersal. In: Clobert J, Danchin E, Dhondt AA, Nichols JD (eds) Dispersal. Oxford University Press, New York, pp 203-216

Ims RA, Yoccoz NG (1997) Studying transfer processes in metapopulations. Emigration, migration and colonization. In: Hanski I, Gilpin ME (eds) Metapopulation biology ecology, genetics and evolution. Academic Press, San Diego, pp 247-265

Isbell LA, Van Vuren D (1996) Differential costs of locational and social dispersal and their consequences for females. Behaviour 133: 1-36

Ives AR, Whitlock MC (2002) Inbreeding and metapopulations. Science 295: 454

Jack KM, Sheller C, Fedigan LM (2011) Social factors influencing natal dispersal in male white-faced capuchins (Cebus capucinus). Am J Primatol 74: 359-365

Jolly GM (1965) Explicit estimates from capture-recapture data with both death and immigration-stochastic model. Biometrika 52: 225-247

Joly M, Zimmermann E (2011) Do solitary foraging nocturnal mammals plan their routes? Biol Lett 7: 638-640

Jones OR, Wang J (2010) COLONY: a program for parentage and sibship inference from multilocus genotype data. Mol Ecol Resour: 551-555

Jones WT (1989) Dispersal distance and the range of nightly movements in Merriam's kangaroo rats. J Mammal 70: 27-34

Jonsen ID, Myers RA, James MC (2006) Robust hierarchical state-space models reveal diel variation in travel rates of migrating leatherback turtles. J Anim Ecol 75: 1046-1057

Kalinowski ST, Taper ML, Marshall TC (2007) Revising how the computer program CERVUS accommodates genotyping error increases success in paternity assignment. Mol Ecol 16: 1099-1106

Kaplan JR, Fontenot MB, Berard J, Manuck SB, Mann JJ (1995) Delayed dispersal and elevated monoaminergic activity in free-ranging rhesus monkeys. Am J Primatol 35: 229-234

Kappeler PM, Fichtel C (2012a) A 15-year perspective on the social organization and life history of sifaka in Kirindy Forest. In: Kappeler PM, Watts DP (eds) Long-term field studies of primates. Springer, Heidelberg, pp 101-121

Kappeler PM, Fichtel C (2012b) Female reproductive competition in Eulemur rufifrons: eviction and reproductive restraint in a plurally breeding Malagasy primate. Mol Ecol 21: 685-698

Kappeler PM, Rasoloarison RM (2003) Microcebus, mouse lemurs, tsidy. In: Goodman SM, Benstead JP (eds) The natural history of Madagascar. The University of Chicago Press, Chicago, pp 1310-1315

Kawecki TJ (2000) The evolution of genetic canalization under fluctuating selection. Evolution 54: 1-12 
Keane B (1990) Dispersal and inbreeding avoidance in the white-footed mouse, Peromyscus leucopus. Anim Behav 40: 143-152

Kempenaers B (2007) Mate choice and genetic quality: a review of the heterozygosity theory. In: Adv Study Behav, vol 37. Academic Press, San Diego, pp 189-278

King R, Morgan BJT, Gimenez O, Brooks SP (2010) Bayesian analysis for population ecology. Chapman \& Hall/ CRC Press, London

Koenig WD, Van Vuren D, Hooge PN (1996) Detectability, philopatry, and the distribution of dispersal distances in vertebrates. Trends Ecol Evol 11: 514-517

Krackow S (2003) Motivational and heritable determinants of dispersal latency in wild male house mice (Mus musculus musculus). Ethology 109: 671-689

Krackow S, Matuschak B (1991) Mate choice for non-siblings in wild house mice: evidence from a choice test and a reproductive test. Ethology 88: 99-108

Kraus C, Eberle M, Kappeler PM (2008) The costs of risky male behaviour: sex differences in seasonal survival in a small sexually monomorphic primate. Proc R Soc B: $1635-1644$

Kyriazakis I, Tolkamp BJ, Emmans G (1999) Diet selection and animal state: an integrative framework. Proceedings of the Nutritional Society 58: 765-772

Lambin X, Aars J, Piertney SB (2001) Dispersal, intraspecific competition, kin competition and kin facilitation: a review of empirical evidence. In: Clobert J, Danchin E, Dhondt AA, Nichols JD (eds) Dispersal. Oxford University Press, New York, pp 110-122

Lampert KP, Rand AS, Mueller UG, Ryan MJ (2003) Fine-scale genetic pattern and evidence for sex-biased dispersal in the túngara frog, Physalaemus pustulosus. Mol Ecol 12: 3325-3334

Langrock R (2011) Some applications of nonlinear and non-Gaussian state-space modelling by means of hidden Markov models. J Appl Stat 38: 2955-2970

Langrock R, MacDonald IL, Zucchini W (2012) Some nonstandard stochastic volatility models and their estimation using structured hidden Markov models. J Empir Financ 19: 147161

Langrock R, Zucchini W (2011) Hidden Markov models with arbitrary dwell-time distributions. Comput Stat Data Anal 55: 715-724

Larsen KW, Boutin S (1994) Movements, survival, and settlement of red squirrel (Tarniasciurus hudsonicils) offspring. Ecology 75: 214-223

Lawson Handley LJ, Perrin N (2007) Advances in our understanding of mammalian sex-biased dispersal. Mol Ecol 16: 1559-1578

Le Galliard J-F, Rémy A, Ims RA, Lambin X (2012) Patterns and processes of dispersal behaviour in arvicoline rodents. Mol Ecol 21: 505-523

Lebreton J, Burnham K, Clobert J, Anderson D (1992) Modeling survival and testing biological hypotheses using marked animals: a unified approach with case studies. Ecol Monogr 62: 67-118

Lehmann L, Perrin N (2003) Inbreeding avoidance through kin recognition: choosy females boost male dispersal. Am Nat 162: 638-652

Leibold MA, Holyoak M, Mouquet N, Amarasekare P, Chase JM, Hoopes MF, Holt RD, Shurin JB, Law R, Tilman D, Loreau M, Gonzalez A (2004) The metacommunity concept: a framework for multi-scale community ecology. Ecol Lett 7: 601-613

Lens LUC, Dhondt AA (1994) Effects of habitat fragmentation on the timing of crested tit Parus cristatus natal dispersal. Ibis 136: 147-152

Lidicker WZ, Stenseth NC (1992) To disperse or not disperse: who does it and why? In: Stenseth NC, Lidicker WZ (eds) Animal dispersal: Small mammals as a model. Chapman \& Hall, London, pp 21-36 
Loew S (1999) Sex-biased dispersal in eastern chipmunks, Tamias striatus. Evol Ecol 13: 557577

Long ES, Diefenbach DR, Rosenberry CS, Wallingford BD (2008) Multiple proximate and ultimate causes of natal dispersal in white-tailed deer. Behav Ecol 19: 1235-1242

Lührs ML, Dammhahn M, Kappeler PM, Fichtel C (2009) Spatial memory in the grey mouse lemur (Microcebus murinus). Anim Cogn 12: 599-609

Lukas D, Clutton-Brock TH (2011) Group structure, kinship, inbreeding risk and habitual female dispersal in plural-breeding mammals. J Evol Biol 24: 2624-2630

Lurz PWW, Garson PJ, Wauters LA (1997) Effects of temporal and spatial variation in habitat quality on red squirrel dispersal behaviour. Anim Behav 54: 427-435

Lutermann H, Schmelting B, Radespiel U, Ehresmann P, Zimmermann E (2006) The role of survival for the evolution of female philopatry in a solitary forager, the grey mouse lemur (Microcebus murinus). Proc R Soc B 273: 2527-2533

Mabry KE, Stamps JA (2008a) Dispersing brush mice prefer habitat like home. Proc R Soc B 275: 543-548

Mabry KE, Stamps JA (2008b) Searching for a new home: decision making by dispersing brush mice. Am Nat 172: 625-634

MacArthur RH, Pianka ER (1966) On optimal use of a patchy environment. Am Nat 100: 603609

MacDonald IL, Raubenheimer D (1995) Hidden Markov models and animal behaviour. Biometrics 37: 701-712

MacDonald IL, Zucchini W (1997) Hidden Markov models and other models for discretevalued time series. Chapman \& Hall, London

Markert JA, Grant PR, Grant BR, Keller LF, Coombs JL, Petren K (2004) Neutral locus heterozygosity, inbreeding, and survival in Darwin's ground finches (Geospiza fortis and G. scandens). Heredity 92: 306-315

Marshall DJ, Bonduriansky R, Bussière LF (2008) Offspring size variation within broods as a bet-hedging strategy in unpredictable environments. Ecology 89: 2506-2517

Martin A, Bateson P (1993) Measuring behaviour. An introductory guide. Cambridge University Press, Cambridge

Maruotti A, Rydén T (2009) A semiparametric approach to hidden Markov models under longitudinal observations. Statistics and Computing 19: 381-393

Massot M, Clobert J, Lorenzon P, Rossi JM (2002) Condition-dependent dispersal and ontogeny of the dispersal behaviour: an experimental approach. J Anim Ecol 71: 253261

Massot M, Huey RB, Tsuji J, van Berkum FH (2003) Genetic, prenatal, and postnatal correlates of dispersal in hatchling fence lizards (Sceloporus occidentalis). Behav Ecol 14: 650-655

Matthysen E (2005) Density-dependent dispersal in birds and mammals. Ecography 28: $403-$ 416

Matthysen E, Currie D (1996) Habitat fragmentation reduces disperser success in juvenile nuthatches Sitta europaea: evidence from patterns of territory establishment. Ecography 19: 67-72

McFarland D (1999) Animal behaviour: psychobiology, ethology and evolution, 3 edn. Longman Scientific and Technical, Harlow

McNamara JM, Houston Al (2009) Integrating function and mechanism. Trends Ecol Evol 24: $670-675$ 
McNutt JW (1996) Sex-biased dispersal in African wild dogs, Lycaon pictus. Anim Behav 52: 1067-1077

Mech LD (1987) Age, season, distance, direction, and social aspects of wolf dispersal from a Minnesota pack. In: Chepko-Sade BD, Halpin ZT (eds) Mammalian dispersal patterns: the effects of social structure on population genetics. The University of Chicago Press, Chicago, pp 55-74

Mech SG, Zollner PA (2002) Using body size to predict perceptual range. Oikos 98: 47-52

Melino A, Turnbull SM (1990) Pricing foreign currency options with stochastic volatility. J Econometrics 45: 239-265

Mestre L, Bonte D (2012) Food stress during juvenile and maternal development shapes natal and breeding dispersal in a spider. Behav Ecol, doi: 10.1093/beheco/ars024

Meylan S, Belliure J, Clobert J, de Fraipont M (2002) Stress and body condition as prenatal and postnatal determinants of dispersal in the common lizard (Lacerta vivipara). Horm Behav 42: 319-326

Mittermeier RA, Louis EE, Richardson M, Schwitzer C, Langrand O, Rylands AB, Hawkins F, Rajaobelina S, Ratsimbazafy J, Rasoloarison R, Roos C, Kappeler PM, Mackinnon J (2010) Lemurs of Madagascar, 3 edn. Conservation International, Arlington

Moore J (1992) Dispersal, nepotism, and primate social behavior. Int J Primatol 13: 361-378

Moore J, Ali R (1984) Are dispersal and inbreeding avoidance related? Anim Behav 32: 94112

Moore J, Ali R (1985) Inbreeding and dispersal: reply to Packer (1985). Anim Behav 33: 13671369

Morales JM, Haydon DT, Frair J, Holsinger KE, Fryxell JM (2004) Extracting more out of relocation data: building movement models as mixtures of random walks. Ecology 85: 2436-2445

Morgan BJT (1976) Markov properties of sequences of behaviours. J R Stat Soc Ser A Stat Soc 25: 31-36

Muniz L, Perry S, Manson JH, Gilkenson H, Gros-Louis J, Vigilant L (2006) Father-daughter inbreeding avoidance in a wild primate population. Curr Biol 16: R156-R157

Murray BG (1967) Dispersal in vertebrates. Ecology 48: 975-978

Murren CJ, Julliard R, Schlichting CD, Clobert J (2001) Dispersal, individual phenotype and phenotypic plasticity. In: Clobert J, Danchin E, Dhondt AA, Nichols JD (eds) Dispersal. Oxford University Press, New York, pp 261-272

Nielsen JF, English S, Goodall-Copestake WP, Wang J, Walling CA, Bateman AW, Flower TP, Sutcliffe RL, Samson J, Thavarajah NK, Kruuk LEB, Clutton-Brock TH, Pemberton JM (2012) Inbreeding and inbreeding depression of early life traits in a cooperative mammal. Mol Ecol 21: 2788-2804

Nilsson J-A, Smith HG (1985) Early fledgling mortality and the timing of juvenile dispersal in the marsh tit Parus palustris. Ornis Scand 16: 293-298

Nilsson J-A, Smith HG (1988) Effects of dispersal date on winter flock establishment and social dominance in marsh tits Parus palustris. J Anim Ecol 57: 917-928

Nunes S (2007) Dispersal and philopatry. In: Wolff JO, Sherman PW (eds) Rodent societies an ecological and evolutionary perspective. The University of Chicago Press, Chicago, pp 150-162

Nunes S, Ha C-DT, Garrett PJ, Mueke E-M, Smale L, Holekamp KE (1998) Body fat and time of year interact to mediate dispersal behaviour in ground squirrels. Anim Behav 55: 605614 
Nunes S, Holekamp KE (1996) Mass and fat influence the timing of natal dispersal in Belding's ground squirrels. J Mammal 77: 807-817

O'Riain MJ, Bennett NC, Brotherton PNM, Mcllrath G, Clutton-Brock TH (2000) Reproductive suppression and inbreeding avoidance in wild populations of co-operatively breeding meerkats (Suricata suricatta). Behav Ecol Sociobiol 48: 471-477

O'Riain MJ, Braude S (2001) Inbreeding versus outbreeding in captive and wild populations of naked mole-rats. In: Clobert J, Danchin E, Dhondt AA, Nichols JD (eds) Dispersal. Oxford University Press, New York, pp 143-154

Oh K, Badyaev A (2008) Evolution of adaptation and mate choice: parental relatedness affects expression of phenotypic variance in a natural population. Evolutionary Biology 35: 111-124

Olson LE, Blumstein DT, Pollinger JR, Wayne RK (2012) No evidence of inbreeding avoidance despite demonstrated survival costs in a polygynous rodent. Mol Ecol 21: 562-571

Overall ADJ, Byrne KA, Pilkington JG, Pemberton JM (2005) Heterozygosity, inbreeding and neonatal traits in Soay sheep on St Kilda. Mol Ecol 14: 3383-3393

Pasinelli G, Schiegg K, Walters JR (2004) Genetic and environmental influences on natal dispersal distance in a resident bird species. Am Nat 164: 660-669

Patterson TA, Basson M, Bravington MV, Gunn JS (2009) Classifying movement behaviour in relation to environmental conditions using hidden Markov models. J Anim Ecol 78: $1113-1123$

Patterson TA, Thomas L, Wilcox C, Ovaskainen O, Matthiopoulos J (2008) State-space models of individual animal movement. Trends Ecol Evol 23: 87-94

Pedersen MW, Patterson TA, Thygesen UH, Madsen H (2011) Estimating animal behavior and residency from movement data. Oikos 120: 1281-1290

Perret M (1990) Influence of social factors on sex ratio at birth, maternal investment and young survival in a prosimian primate. Behav Ecol Sociobiol 27: 447-454

Perrin N, Goudet J (2001) Inbreeding, kinship, and the evolution of natal dispersal. In: Clobert J, Danchin E, Dhondt AA, Nichols JD (eds) Dispersal. Oxford University Press, New York, pp 123-142

Perrin N, Mazalov V (1999) Dispersal and inbreeding avoidance. Am Nat 154: 282-292

Perrin N, Mazalov V (2000) Local competition, inbreeding, and the evolution of sex-biased dispersal. Am Nat 155: 116-127

Petrie M, Kempenaers B (1998) Extra-pair paternity in birds: explaining variation between species and populations. Trends Ecol Evol 13: 52-58

Pusey A (1988) Reply from A. E. Pusey. Trends Ecol Evol 3: 145-146

Pusey A, Wolf M (1996) Inbreeding avoidance in animals. Trends Ecol Evol 11: 201-206

Pusey AE (1987) Sex-biased dispersal and inbreeding avoidance in birds and mammals. Trends Ecol Evol 2: 295-299

Pusey AE, Packer C (1987) Dispersal and philopatry. In: Smutts BB, Cheney DL, Seyfarth RM, Wrangham RW, Struhsaker TT (eds) Primate societies. The University of Chicago Press, Chicago, pp 250-266

Quirici V, Faugeron S, Hayes L, Ebensperger L (2011) The influence of group size on natal dispersal in the communally rearing and semifossorial rodent, Octodon degus. Behav Ecol Sociobiol 65: 787-798

Rabiner LR (1989) A tutorial on hidden Markov models and selected applications in speech recognition. Proceedings of the IEEE 7: 257-286

Radespiel U (2000) Sociality in the gray mouse lemur (Microcebus murinus) in Northwestern Madagascar. Am J Primatol 51: 21-40 
Radespiel U, Lutermann H, Schmelting B, Bruford MW, Zimmermann E (2003) Patterns and dynamics of sex-biased dispersal in a nocturnal primate, the grey mouse lemur, Microcebus murinus. Anim Behav 65: 707-719

Radespiel U, Zimmermann E (2001) Female dominance in captive gray mouse lemurs (Microcebus murinus). Am J Primatol 54: 181-192

Radespiel U, Zübeyde S, Zimmermann E, Bruford MW (2001) Sociogenetic structure in a freeliving nocturnal primate population: sex-specific differences in the grey mouse lemur (Microcebus murinus). Behav Ecol Sociobiol 50: 493-502

Rajpurohit LS, Sommer VJ (1993) Juvenile male emigration from natal one-male troops in Hanuman langurs. In: Pereira ME, Fairbanks LA (eds) Juvenile primates. Oxford University Press, New York, pp 86-103

Rasoloarison RM, Goodman S, Ganzhorn JU (2000) Taxonomic revision of mouse lemur (Microcebus) in the Western portions of Madagascar. Int J Primatol 21: 963-1019

Rasoloarison RM, Rasolonandrasana BPN, Ganzhorn JU, Goodman SM (1995) Predation on vertebrates in the Kirindy Forest, Western Madagascar. Ecotropica 1: 59-65

Réale D, Reader SM, Sol D, McDougall PT, Dingemanse NJ (2005) Integrating animal temperament within ecology and evolution. Biol Rev Camb Philos Soc 142: 291-318

Reed JM, Dobson AP (1993) Behavioural constraints and conservation biology: conspecific attraction and recruitment. Trends Ecol Evol 8: 253-256

Rice WR (1990) A consensus combined $p$-value test and the family-wide significance of component tests. Biometrics 46: 303-308

Ridley AW, Hereward JP, Daglish GJ, Raghu S, Collins PJ, Walter GH (2011) The spatiotemporal dynamics of Tribolium castaneum (Herbst): adult flight and gene flow. Mol Ecol 20: 1635-1646

Riters LV, Eens M, Pinxten R, Duffy DL, Balthazart J, Ball GF (2000) Seasonal changes in courtship song and the medial preoptic area in male European starlings (Sturnus vulgaris). Horm Behav 38: 250-261

Robbins AM, Stoinski TS, Fawcett KA, Robbins MM (2009) Does dispersal cause reproductive delays in female mountain gorillas? Behaviour 146: 525-549

Rodgers AR, Carr AP, Beyer HL, Smith L, Kie JG (2007) HRT: home range tools for ArcGIS Version 1.1. Ontario Ministry of Natural Resources, Centre for Northern Forest Ecosystem Research, Thunder Bay Ontario, Canada

Roff DA (2002) Inbreeding depression: tests of the overdominance and partial dominance hypotheses. Evolution 56: 768-775

Roff DA, Fairbairn DJ (2001) The genetic basis of dispersal and migration, and its consequences for the evolution of correlated traits. In: Clobert J, Danchin E, Dhondt AA, Nichols JD (eds) Dispersal. Oxford University Press, New York, pp 191-202

Rogers LL (1987) Factors influencing dispersal in the black bear. In: Chepko-Sade BD, Halpin ZT (eds) Mammalian dispersal patterns: the effects of social structure on population genetics. The University of Chicago Press, Chicago, pp 75-84

Ronce $\mathrm{O}$ (2007) How does it feel to be like a rolling stone? Ten questions about dispersal evolution. Annu Rev Ecol Evol Syst 38: 231-253

Ronce O, Olivieri I, Clobert J, Danchin E (2001) Perspectives on the study of dispersal evolution. In: Clobert J, Danchin E, Dhondt AA, Nichols JD (eds) Dispersal. Oxford University Press, New York, pp 341-357

Rood JP (1987) Dispersal and intertroup transfer in the dwarf mongoose. In: Chepko-Sade BD, Halpin ZT (eds) Mammalian dispersal patterns: the effects of social structure on population genetics. The University of Chicago Press, Chicago, pp 85-103 
Roper TJ, Ostler JR, Conradt L (2003) The process of dispersal in badgers Meles meles. Mammal Rev 33: 314-318

Rousset F, Gandon S (2002) Evolution of the distribution of dispersal distance under distance-dependent cost of dispersal. J Evol Biol 15: 515-523

Roy J, Yannic G, Côté SD, Bernatchez L (2012) Negative density-dependent dispersal in the American black bear (Ursus americanus) revealed by noninvasive sampling and genotyping. Ecology and Evolution 2: 525-537

Royer F, Fromentin J-M, Gaspar P (2005) A statespace model to derive bluefin tuna movement and habitat from archival tags. Oikos 109: 473-484

Savage A, Giraldo LH, Soto LH, Snowdon CT (1996) Demography, group composition, and dispersal in wild cotton-top tamarin (Saguinus oedipus) groups. Am J Primatol 38: 85100

Schilling A (1979) Olfactory communication in prosimians. In: Doyle GA, Martin AD (eds) The study of prosimian behavior. Academic Press, New York, pp 461-542

Schmid J (1998) Tree holes used for resting by gray mouse lemurs (Microcebus murinus) in Madagascar: insulation capacities and energetic consequences. Int J Primatol 19: 797-809

Schmid J (1999) Sex-specific differences in activity patterns and fattening in the grey mouse lemur (Microcebus murinus) in Madagascar. J Mammal 80: 749-757

Schmid J, Kappeler PM (1998) Fluctuating sexual dimorphism and differential hibernation by sex in a primate, the gray mouse lemur (Microcebus murinus). Behav Ecol Sociobiol 43: $125-132$

Schoof VAM, Jack KM, Isbell LA (2009) What traits promote male parallel dispersal in primates? Behaviour 146: 701-726

Schwartz OA, Armitage KB, Van Vuren D (1998) A 32-year demography of yellow-bellied marmots (Marmota flaviventris). J Zool 246: 337-346

Schwensow N, Eberle M, Sommer S (2008) Compatibility counts: MHC-associated mate choice in a wild promiscuous primate. Proc R Soc B 275: 555-564

Scott SL (2002) Bayesian methods for hidden Markov models: recursive computing in the 21st century. JASA 97: 337-351

Seber GAF (1965) A note on the multiple-recapture census. Biometrika 52: 249-259

Selonen V, Hanski IK (2010) Condition-dependent, phenotype-dependent and geneticdependent factors in the natal dispersal of a solitary rodent. J Anim Ecol 79: 10931100

Seltman HJ (2002) Hidden Markov models for analysis of biological rhythm data. In: Gatsonis C, Kass RE, Carlin B, Carriquiry A, Gelman A, Verdinelli I, West M (eds) Case studies in Bayesian statistics, vol 5. Springer, New York, pp 398-406

Shields WM (1987) Dispersal and mating system: investigating their causal connections. In: Chepko-Sade BD, Halpin ZT (eds) Mammalian dispersal patterns - the effects of social structure on population genetics. The University of Chicago Press, Chicago, pp 3-24

Smale L, Nunes S, Holekamp KE, Slater PJB, Rosenblatt JS, Snowdon CT, Milinski M (1997) Sexually dimorphic dispersal in mammals: patterns, causes, and consequences. In: Adv Study Behav, vol 26. Academic Press, San Diego, pp 181-250

Smith AT (1974) The distribution and dispersal of pikas: consequences of insular population structure. Ecology 55: 1112-1119

Smith AT (1987) Population structure of pikas: dispersal versus philopatry. In: Chepko-Sade BD, Halpin ZT (eds) Mammalian dispersal patterns: the effects of social structure on population genetics. The University of Chicago Press, Chicago, pp 127-142 
Sorg JP, Ganzhorn JU, Kappeler PM (2003) Forestry and research in the Kirindy Forest / Centre de Formation Professionelle Forestière. In: Goodman SM, Benstead JP (eds) The natural history of Madagascar. The University of Chicago Press, Chicago, pp 1512-1519

Stamps JA (2001) Habitat selection by dispersers: integrating proximate and ultimate approaches. In: Clobert J, Danchin E, Dhondt AA, Nichols JD (eds) Dispersal. Oxford University Press, New York, pp 230-242

Stamps JA (2006) The silver spoon effect and habitat selection by natal dispersers. Ecol Lett 9: $1179-1185$

Steen H (1994) Low survival of long distance dispersers of the root vole (Microtus oeconomus). Ann Zool Fenn 31: 271-274

Stockley P, Searle JB, Macdonald DW, Jones CS (1993) Female multiple mating behaviour in the common shrew as a strategy to reduce inbreeding. Proc R Soc B 254: 173-179

Storm GL, Andrews RL, Phillips RL, Bishop RA, Siniff DB, Tester JR (1976) Morphology reproduction, dispersal, and mortality of midwestern red fox populations. Wildl Monogr 49: 1-82

Strier KB (1994) Myth of the typical primate. Am J Phys Anthropol 37: 233-271

Stumpf RM, Thompson ME, Muller MN, Wrangham RW (2009) The context of female dispersal in Kanyawara chimpanzees. Behaviour 146: 629-656

Sutherland GD, Harestad AS (2000) Scaling of natal dispersal distances in terrestrial birds and mammals. Conservation Ecology 4: 16-52

Trefilov A, Berard J, Krawczak M, Schmidtke J (2000) Natal dispersal in rhesus macaques is related to serotonin transporter gene promoter variation. Behav Genet 30: 295-301

Tregenza T, Wedell N (2000) Genetic compatibility, mate choice and patterns of parentage: invited review. Mol Ecol 9: 1013-1027

Trivers RL (1972) Parental investment and sexual selection. In: Campbell B (ed) Sexual selection and the descent of man 1871-1971. Aldine, Chicago, pp 136-179

Tucker AD, McCallum HI, Limpus CJ, McDonald KR (1998) Sex-biased dispersal in a long-lived polygynous reptile (Crocodylus johnstoni). Behav Ecol Sociobiol 44: 85-90

Tucker BC, Anand M (2005) On the use of stationary versus hidden Markov models to detect simple versus complex ecological dynamics. Ecol Model 185: 177-193

Van Dyck H, Baguette M (2005) Dispersal behaviour in fragmented landscapes: routine or special movements? Basic Appl Ecol 6: 535-545

Van Noordwijk MA, Arora N, Willems EP, Dunkel LP, Amda RN, Mardianah N, Ackermann C, Krützen M, van Schaik CP (2012) Female philopatry and its social benefits among Bornean orangutans. Behav Ecol Sociobiol 66: 823-834

Van Noordwijk MA, Van Schaik CP (2001) Career moves: transfer and rank challenge decisions by male long-tailed macaques. Behaviour 138: 359-395

Van Oosterhout C, Hutchinson WF, Wills DPM, Shipley P (2004) Micro-checker: software for identifying and correcting genotyping errors in microsatellite data. Mol Ecol Notes 4: 535-538

Van Vuren D, Armitage KB (1994) Survival of dispersing and philopatric yellow-bellied marmots: what is the cost of dispersal? Oikos 69: 179-181

Vogler C, Metaxas D (1997) Adapting hidden Markov models for ASL recognition by using threedimensional computer vision methods. In: IEEE International Conference on Systems, Man, and Cybernetics. Computational Cybernetics and Simulation, vol 1, Orlando Florida, pp 156-161 
Vuilleumier S, Perrin N (2006) Effects of cognitive abilities on metapopulation connectivity. Oikos 113: 139-147

Wang J (2007) Triadic IBD coefficients and applications to estimating pairwise relatedness. Genet Res 89: 135-153

Wang J (2011) Coancestry: a program for simulating, estimating and analysing relatedness and inbreeding coefficients. Mol Ecol Resour 11: 141-145

Wang P, Puterman ML (2001) Analysis of longitudinal data of epileptic seizure: a two state hidden Markov approach. Biometrical J 43: $941-962$

Waser PM (1985) Does competition drive dispersal? Ecology 66: 1170-1175

Waser PM, Austad SN, Keane B (1986) When should animals tolerate inbreeding? Am Nat 128: 529-537

Waser PM, Berning ML, Pfeifer A (2012) Mechanisms of kin discrimination inferred from pedigrees and the spatial distribution of mates. Mol Ecol 21: 554-561

Wauters L, Bijnens L, Dhondt AA (1993) Body mass at weaning and juvenile recruitment in the red squirrel. J Anim Ecol 62: 280-286

Wauters LA, Lens L, Dhondt AA (1995) Variation in territory fidelity and territory shifts among red squirrel, Sciurus vulgaris, females. Anim Behav 49: 187-193

Welch G, Bishop G (1995) An introduction to the Kalman filter. In: UNC-CH Computer Science Technical Report, vol 95-041. UNC-CH - Department of Computer Science

Welch LR (2003) Hidden Markov models and the Baum-Welch algorithm. IEEE Information Theory Society Newsletter 53: 10-13

White GC, Burnham KP (1999) Program MARK: survival estimation from populations of marked animals. Bird Study 46: 120-139

Whitten PI, Brockman DK, Stavisky RC (1998) Recent advances in noninvasive techniques to monitor hormone-behavior interactions. Yearb Phys Anthropol 41: 1-23

Widdig A (2007) Paternal kin discrimination: the evidence and likely mechanisms. Biol Rev 82: 319-334

Wiens JA (2001) The landscape context of dispersal. In: Clobert J, Danchin E, Dhondt AA, Nichols JD (eds) Dispersal. Oxford University Press, New York, pp 96-109

Wiens JA, Zitzmann A (2003) Social structure of the solitary slow loris Nycticebus coucang (Lorisidae). J Zool 261: 35-46

Wimmer B, Tautz D, Kappeler PM (2002) The genetic population structure of the gray mouse lemur (Microcebus murinus). Behav Ecol Sociobiol 52: 166-175

Winnie Jr. J, Christianson D, Creel S, Maxwell B (2006) Elk decision-making rules are simplified in the presence of wolves. Behav Ecol Sociobiol 61: 277-289

Winters JB, Waser PM (2003) Gene dispersal and outbreeding in a philopatric mammal. Mol Ecol 12: 2251-2259

Wolff JO (1992) Parents suppress reproduction and stimulate dispersal in opposite-sex juvenile white-footed mice. Nature 359: 409-410

Wolff JO (1994) More on juvenile dispersal in mammals. Oikos 71: 349-352

Wrogemann D, Radespiel U, Zimmermann E (2001) Comparison of reproductive characteristics and changes in body weight between captive populations of rufous and gray mouse lemurs. Int J Primatol 22: 91-108

Yackulic CB, Blake S, Deem S, Kock M, Uriarte M (2011) One size does not fit all: flexible models are required to understand animal movement across scales. J Anim Ecol 80: 1088-1096

Yoder JM, Marschall EA, Swanson DA (2004) The cost of dispersal: predation as a function of movement and site familiarity in ruffed grouse. Behav Ecol 15: 469-476 


\section{REFERENCES}

Zeh JA, Zeh DW (2006) Outbred embryos rescue inbred half-siblings in mixed-paternity broods of live-bearing females. Nature 439: 201-203

Zollner PA, Lima SL (1999) Search strategies for landscape-level interpatch movements. Ecology 18: 1019-1030

Zollner PA, Lima SL (2006) Behavioral tradeoffs when dispersing across a patchy landscape. Oikos 108: 219-230

Zucchini W, MacDonald IL (2009) Hidden Markov models for time series: an introduction using R. Chapman \& Hall, London

Zucchini W, Raubenheimer D, MacDonald IL (2008) Modeling time series of animal behavior by means of a latent-state model with feedback. Biometrics 64: 807-815 